\title{
Reflux, Aspiration and Respiratory Disease in Dogs: Clinical Consideration and Advances in Aerodigestive Diagnostics
}

\author{
A Dissertation \\ Presented to the Faculty of the Graduate School \\ at the University of Missouri-Columbia
}

In Partial Fulfillment

of the Requirements for the Degree of

PhD in Veterinary Pathobiology

by
MEGAN ELIZABETH GROBMAN
Carol R. Reinero, Dissertation Supervisor

July 2019 
The undersigned, appointed by the dean of the Graduate School, have examined the dissertation entitled:

\section{Reflux, Aspiration and Respiratory Disease in Dogs: Clinical Consideration and Advances in Aerodigestive Diagnostics}

Presented by Megan Grobman, a candidate for the degree of doctor of philosophy, and herby certify that, in their opinion, it is worthy of acceptance.

Professor Carol R. Reinero

$\overline{\text { Associate Professor Teresa Lever }}$

Assistant Professor Charles Maitz

Senior Research Associate Hansjörg Rindt 


\section{DEDICATION}

I would like to dedicate this PhD dissertation to Jason. He had no idea what he was signing up for and if worry shaved years off a man's life, he would not have lived to see

30. Thank you Handsome, you are the best decision I have ever made. 


\section{ACKNOWLEDGMENTS}

I have been privileged throughout my training to have had the guidance of exceptional individuals. These people have become my example for what it means to be a doctor, teacher, scientist, and friend. We should all be so lucky.

To my committee: Carol, Teresa, Hans, and Charlie. Thank you for all your support and guidance throughout this process. Your advice and expertise have been invaluable.

To Carol, Leah, and Amy. You deserve an amazing amount of credit for your remarkable enthusiasm, mentorship, and tolerance for my terrible jokes. You showed me the kind of clinician and scientist I want to be. I am privileged to count you as mentors, and friends.

To Shawn and Randi. I knew when I was 5 that I wanted to be a doctor and scientist. Two and half years ago, I learned I could be something else too. You have no idea how far your reach goes. It has meant the world.

To the Nerd Crew. Thank you for your endless humor, support, and cake. Our jokes will never not be funny. Say the word and I will be there with a shovel, a "can do" attitude and not a whole lot of questions. "Un guanaco"

Finally, to Rance. This is all your fault. Thank you for showing me everything I really needed to know about elevator doors.

This would not have happened without you all.

Now to pay it forward.

$$
\text { 1-2-3 "clap" }
$$




\section{TABLE OF CONTENTS}

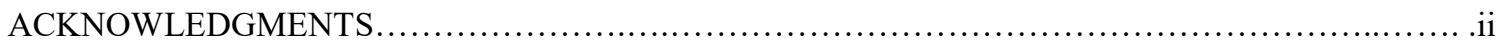

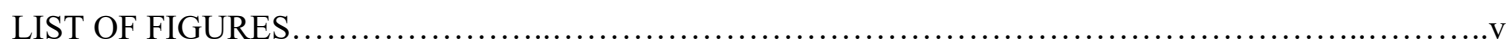

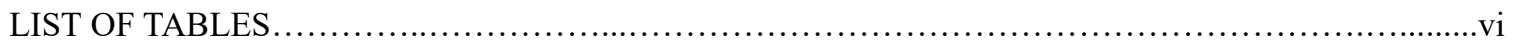

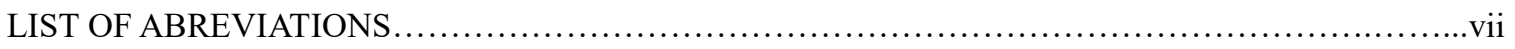

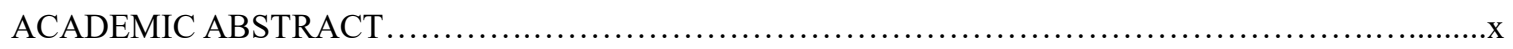

Chapter

1. AERODIGESTIVE DISEASE: PATHOPHYSIOLOGY, PATHOGENESIS AND THE NEED

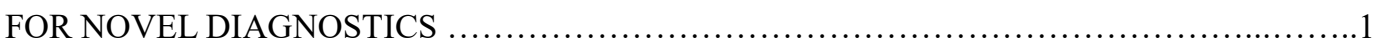

2. AERODIGESTIVE DISORDERS IN DOGS EVALUATED FOR COUGH USING RESPIRATORY FLUOROSCOPY AND VIDEOFLUOROSCOPY SWALLOW STUDIES ......23

3. VIDEOFLUOROSCOPIC SWALLOW STUDY CHARACTERIZATION OF LOWER ESOPHAGEAL SPHINCTER ACHALASIA SYNDROME (LES-AS) IN DOGS.

4. MECHANICAL DILATION AND BOTULINUNM TOXIN A (BTA) INJECTIONS FOR TREATMENT OF LOWER ESOPHAGEAL SPHINCTER ACHALASIA-LIKE SYNDROME

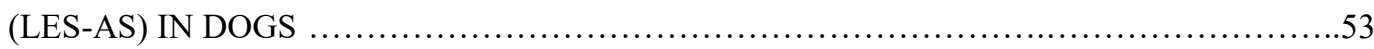

5. DOCUMENTING SILENT REFLUX AND MICROASPIRATION EVENTS USING NUCLEAR

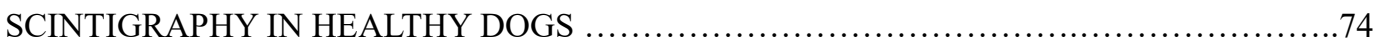

6. PROTEOMIC BIOMARKERS IN REGURGITATION, VOMITING, AND COUGH: PROTEOMIC CHARACTERIZATION OF CANINE GASTRIC FLUID BY LIQUID CHROMATOGRAPHY MASS SPECTROMETRY (LC-MS) ....................................86

7. DISCRIMINATION BETWEEN RESPIRATORY AND NON-RESPIRATORY SOUND WAVEFORMS IN DOGS USING ACOUSTIC WAVE RECORDINGS: AN OBJECTIVE

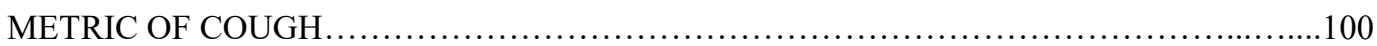

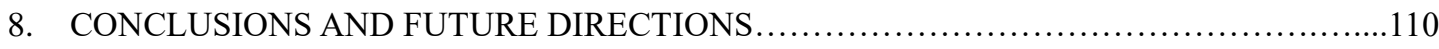

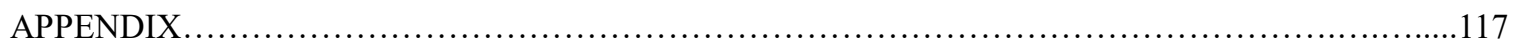

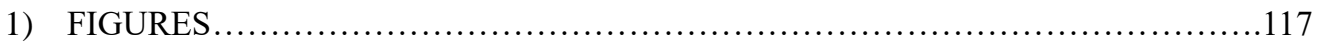

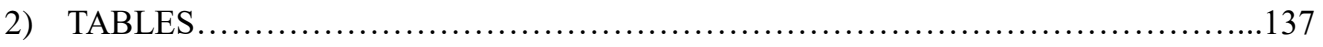


BIBLIOGRAPHY

161

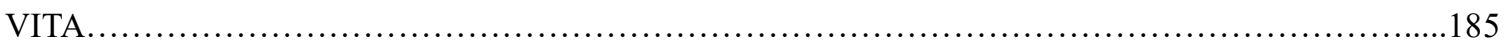




\section{LIST OF FIGURES}

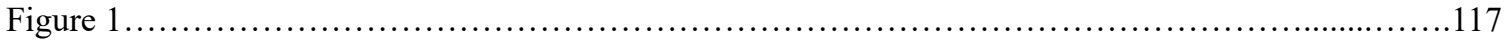

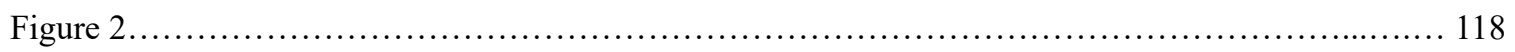

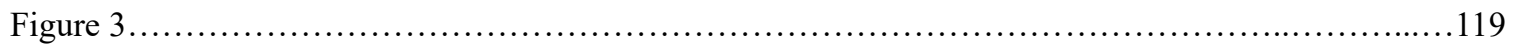

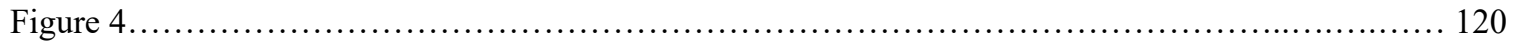

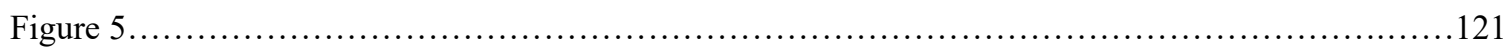

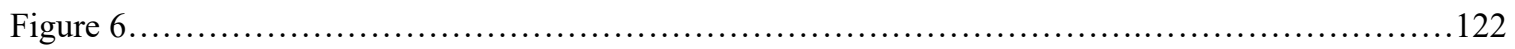

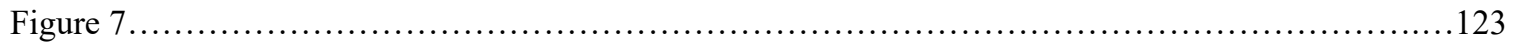

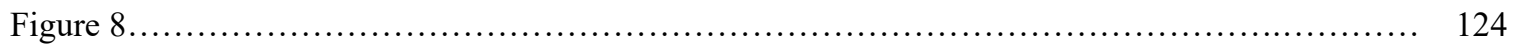

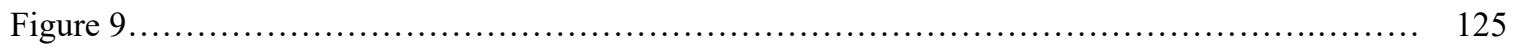

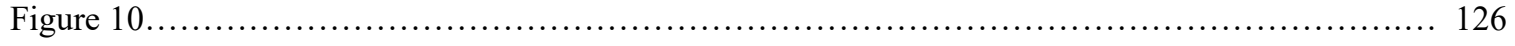

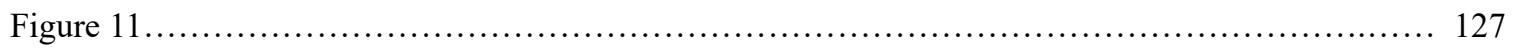

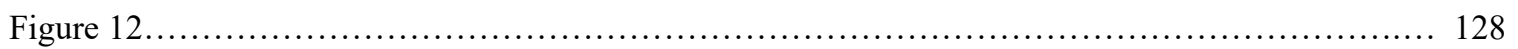

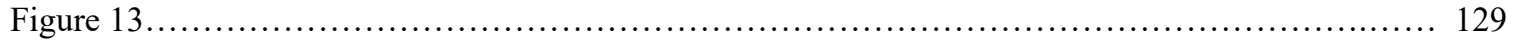

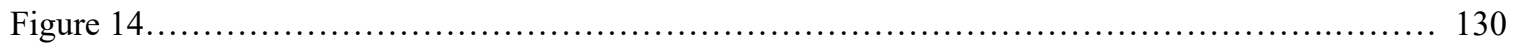

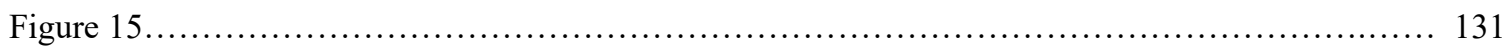

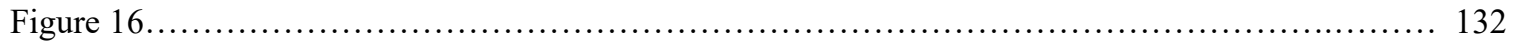

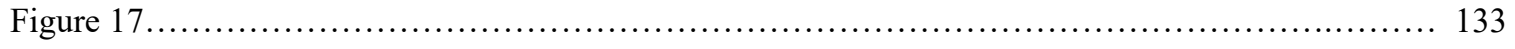

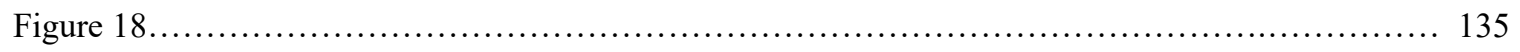

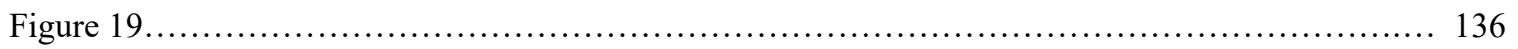




\section{LIST OF TABLES}

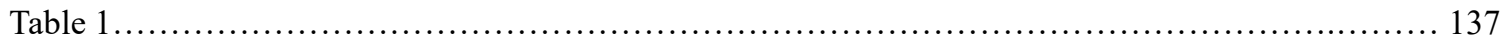

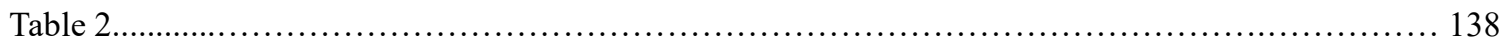

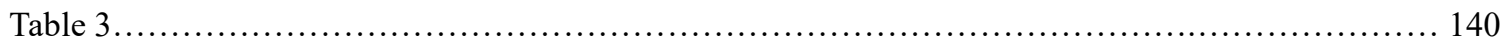

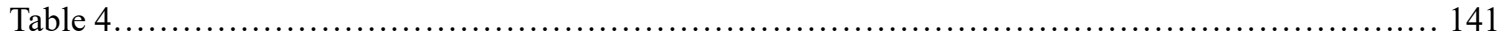

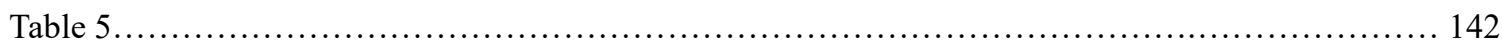

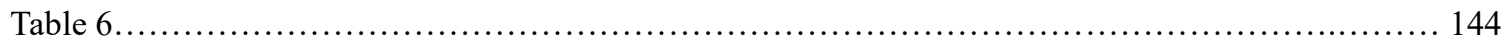

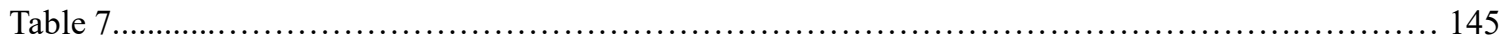

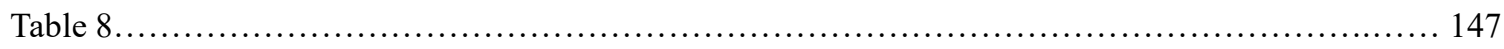

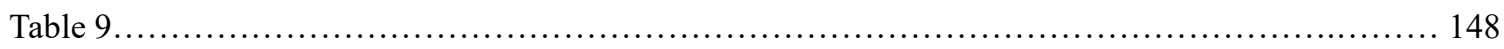

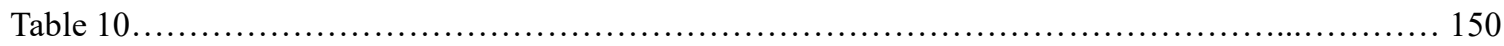

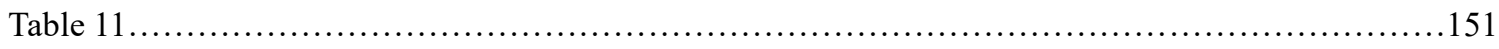

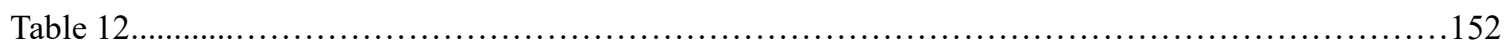

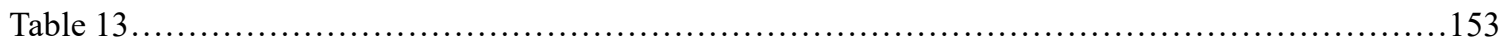

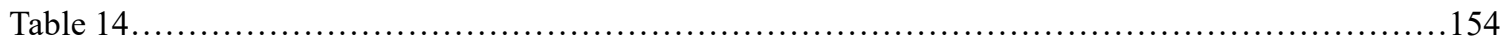

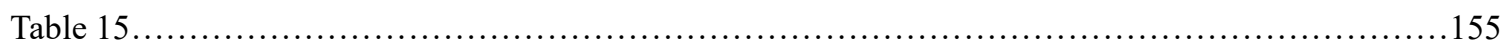

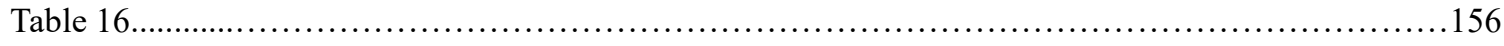

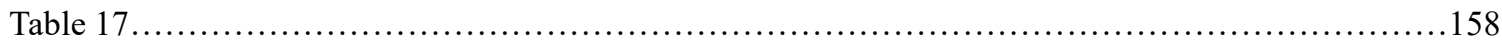

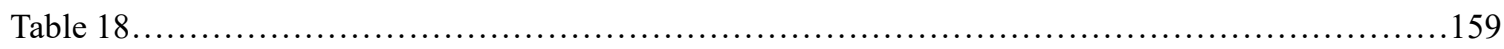

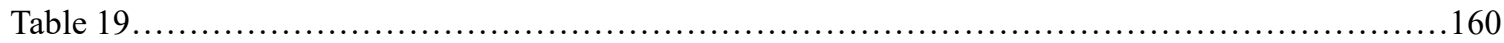




\section{LIST OF ABBREVIATIONS}

1. Acoustic behaviors $(\mathrm{AB})$

2. Acute respiratory distress syndrome (ARDS)

3. Aerodigestive disease(s) (AeroD)

4. Aerophagia (Aero)

5. Analysis of Similarity (ANOSIM)

6. Arytenoids (Aryt)

7. Aspiration associated respiratory syndromes (AARS)

8. Aspiration pneumonia (AP)

9. Body condition score (BCS)

10. Body weight (BW)

11. Botulinum toxin A (BTA)

12. Brachycephalic airway syndrome (BAS)

13. Bronchiectasis (BE)

14. Bronchoalveolar lavage fluid (BALF)

15. Bronchomalacia (BM)

16. Canine chronic bronchitis (CCB)

17. Chronic obstructive pulmonary disease (COPD)

18. Colloidal $99 \mathrm{~m}$-technetium phytate $\left({ }^{99} \mathrm{TcP}\right)$

19. Cricopharyngeus (CP)

20. Decibel (dB)

21. Diagnosis (Dx)

22. Distal $3^{\text {rd }}$ of the esophagus (E1)

23. Distal esophageal diameter (DeD)

24. Eosinophilic bronchitis (EB)

25. Epiglottic retroversion (ER)

26. Epiglottis (E)

27. Esophageal hypomotility $(\mathrm{EH})$

28. Esophageal peristalsis (E-P)

29. Extra-esophageal reflux (EER)

30. Extra-esophageal reflux disease (EERD)

31. Gastric $(\mathrm{G})$

32. Gastric fluid (GF)

33. Gastroesophageal reflux (GER)

34. Gastroesophageal reflux disease (GERD)

35. Gastrointestinal (GI)

36. Hertz (Hz)

37. Hiatal hernia $(\mathrm{HH})$

38. High-resolution manometry (HRM)

39. Hypoplastic trachea (HT)

40. Interquartile Range (IQR)

41. Laryngeal (Lar) 
42. Laryngeal obstruction/defect (LO/D)

43. Laryngeal paralysis (LarPar)

44. Left lung field (LL)

45. Liquid chromatography mass spectrometry (LC-MS)

46. Lower esophageal sphincter (LES)

47. Lower esophageal sphincter achalasia-like syndrome (LES-AS)

48. Main-stem bronchi (MSB)

49. Mega-becquerels (MBq)

50. Megaesophagus (ME)

51. Middle $3^{\text {rd }}$ of the esophagus (E2)

52. Monocyte chemoattractant protein-1 (MCP-1)

53. Myasthenia gravis (MG)

54. Nasopharyngeal reflux (NPR)

55. No $(\mathrm{N})$

56. Normal (Norm)

57. Normalized spectral abundance factor (NSAF)

58. Nucleus ambiguous (AMB)

59. Nucleus tractus solitarius (NTS)

60. Oropharynx/oropharyngeal (OP)

61. Parallel Accumulation Serial Fragmentation (PASEF)

62. Penetration-Aspiration (A-P)

63. Pharyngeal contraction (P-cont)

64. Pharyngeal hypomotility $(\mathrm{PH})$

65. Pharyngeal spasticity (PS)

66. Pharynx (P)

67. Principal component analysis (PCA)

68. Proton pump inhibitors (PPIs)

69. Proximal $3^{\text {rd }}$ of the esophagus (E3)

70. Proximal esophagus (Prox E)

71. Pulmonary parenchymal (PP)

72. Radiographic diagnosis of AP (AP (rad))

73. Radiographic evidence of AP with confirmed secondary bacterial infection (AP (bact))

74. Radiographic evidence of AP without a secondary bacterial infection (AP (sterile))

75. Reflexive pharyngeal swallow (RPS)

76. Regions-of-interest (ROI)

77. Respiratory central pattern generator ( $\mathrm{rCPG})$

78. Respiratory fluoroscopy (RF)

79. Right lung field (RL)

80. Small non-acidified gastric particles (SNAPs)

81. Swallow central pattern generator (swCPG)

82. Throat clear (TC) 
83. Time-activity-curves (TAC)

84. Transient receptor potential ankyrin 1 (TRPA1)

85. Transient receptor potential vanilloid 1 (TRPV1)

86. Trapped ion mobility spectrometry (tims)

87. Tumor necrosis factor alpha (TNFa)

88. University of Missouri Veterinary Health Center (MU-VHC)

89. Upper esophageal sphincter (UES)

90. Videofluoroscopic swallow studies (VFSS)

91. Visual analogue scale (VAS)

92. Yes (Y) 


\begin{abstract}
The aspiration of gastric contents into the respiratory tract has been associated with the development and progression of multiple respiratory diseases in humans. In veterinary medicine the term "aspiration" is considered synonymous with "aspiration pneumonia" which, while frequently encountered, does not accurately reflect the breadth of aspiration associated respiratory syndromes (AARS). In the clinical veterinary literature, the effect of alimentary dysfunction on respiratory disease (aerodigestive disease) is rarely investigated despite evidence in the human literature, animal models, and some studies and case reports linking alimentary and respiratory disease in clinical small animal patients. Aerodigestive disease is rarely investigated in veterinary medicine due to lack of clinical recognition and limitations in available diagnostics. Furthermore, AARS may be precipitated by several potentially occult alimentary disorders. Current methods of investigating aerodigestive diseases in veterinary patients are limited by sensitivity, specificity, potential for bias, cost, and availability. This necessitates investigations into advanced diagnostics to identify a potentially underrecognized patient population with AARS. Additionally, similarities in anatomy, physiology, and several pathologic disorders between dogs and humans, make experimental and naturally occurring canine models integral to translational research. Thus, evaluating dogs with aerodigestive disease may represent an area of substantial clinical relevance in human as well as veterinary medicine.
\end{abstract}




\section{CHAPTER 1}

\section{AERODIGESTIVE DISEASE: PATHOPHYSIOLOGY, PATHOGENESIS AND THE NEED FOR NOVEL DIAGNOSTICS}

\section{Introduction}

Aerodigestive disorders in people encompass a wide range of conditions reflecting the complex interrelationship between swallowing and respiration. That these two systems should be intimately related should come as no surprise given their common embryological origins, anatomy and innervation.(1) In humans, aerodigestive diseases have been implicated in the pathogenesis and progression of several acute and chronic respiratory conditions. For example, gastroesophageal reflux (GER) is a frequent source of acute and chronic pulmonary disease in human medicine with a prevalence of $50 \%$ in patients with chronic cough, asthma and chronic obstructive pulmonary disease (COPD).(2-6) GER and extra-esophageal reflux (EER) are defined as the movement of gastric contents into the esophagus and from the esophagus to the pharynx respectively. Clinical signs resulting from GER and EER are considered gastroesophageal reflux disease (GERD) and extra-esophageal reflux disease (EERD) respectively. These conditions represent a diagnostic challenge because many patients present with an absence of gastrointestinal signs.(7-9) Despite the prevalence of GER in the aforementioned respiratory conditions, up to $45 \%$ of humans with confirmed GERD are asymptomatic for alimentary tract signs.(10) This makes clinical recognition of the relationship between the alimentary and respiratory tracts critically important as uncontrolled reflux in humans is known to lead to progression of respiratory disease and exacerbations of clinical signs, and increase patient morbidity and treatment costs.(11) 
Treatment of GERD and EERD has been demonstrated to reduce the frequency of disease exacerbations and slow the rate of decline in lung function in humans with COPD.(2)

Although aspiration pneumonia is the best recognized example of aerodigestive disease in dogs, the spectrum of possible conditions associated with reflux with or without aspiration is far broader.(12) Animal models and clinical case reports and case series have demonstrated bronchoconstriction, laryngeal dysfunction, and microaspiration in response to experimental or naturally occurring reflux.(12-16) A case series in dogs treated for gastrointestinal tract disease prior to brachycephalic airway surgery found a decreased complication rate and improved prognosis.(17) This suggests that a subpopulation dogs with respiratory disease, like humans, have reflux and may respond to treatment targeting the gastrointestinal tract.

Advanced diagnostics available for the detection of reflux and aspiration associated respiratory syndromes (AARS) in humans include protein biomarkers, high resolution impedance manometry, and ambulatory $\mathrm{pH}$ monitoring. These are not routinely used in veterinary medicine due to limitations in sensitivity, specificity, potential for bias, expense, necessity for clinical expertise, and in some cases extremely limited availability. $(8,18-20)$ This necessitates investigation into more readily available advanced diagnostics to identify patients with ARRS. This review will provide an overview of respiratory and swallow coordination, aspiration associated airway inflammation, risk factors for aspiration, AARS recognized in veterinary medicine, the currently available diagnostics for identification of aerodigestive disease in veterinary 
patients, as well as areas of future interest.

\section{Physiology of Swallowing}

Swallowing is both a feeding behavior and an airway protective behavior. The latter occurs by clearing material from the pharynx and disposing of material removed from the airways by mucociliary clearance.(21) A swallow is classically divided into three sequential phases; the oral/preparatory phase, pharyngeal phase, and esophageal phase. The oral/preparatory phase is largely voluntary and responsible for prehension, mastication, and conduction of a formed food bolus to the vallecullae located between the elevated epiglottis and base of the tongue. $(22,23)$

Unlike the oral/preparatory phase, the pharyngeal and esophageal phases of swallowing are involuntary and irreversible once triggered. The pharyngeal swallow may occur spontaneously (reflexive swallow) or be triggered by mechanical or chemical stimulation of the pharynx and larynx.(21, 24) Stimulation of laryngeal and pharyngeal structures is relayed by the cranial laryngeal nerve, and to a lesser degree the glossopharyngeal (CN 9) and recurrent laryngeal nerves to the brainstem and nucleus tractus solitarius (NTS) and nucleus ambiguous (AMB) to initiate downstream contraction of the pharyngeal and mylohyoid muscles which propel the bolus through the upper esophageal sphincter (UES).(22, 23) The cranial laryngeal and recurrent laryngeal nerve are branches of the vagus nerve (CN 10).

Pharyngeal stimulation triggers the esophageal phase of swallow by triggering a primary esophageal contraction. Subsequent contraction of the esophageal musculature conducts the bolus into the stomach through an open lower esophageal sphincter (LES) followed by LES closure to prevent reflux. Normal esophageal peristalsis may be 
modified during multiple rapid swallows where primary esophageal peristalsis is inhibited and the LES remains relaxed until the end of the last pharyngeal swallow before returning to baseline. (25) It is important to note that motor endplates of esophageal skeletal muscle differ from skeletal muscle elsewhere in the body and reflects a complex point of integration between extrinsic vagal cholinergic, intrinsic nitrergic, peptidergic, and monaminergic neurons. Co-innervation allows modulation of esophageal skeletal muscle by myenteric neurons and may reflect a mechanism for common diseases, such as megaesophagus, that are observed between species despite differences in esophageal muscle composition. Different species show significant variability in the proportion of skeletal to smooth muscle in the body of the esophagus. The canine esophagus is composed entirely of skeletal muscle compared to where smooth muscle is the dominant muscle type, particularly in the distal esophagus. (26)

The opening of the LES in response to a pharyngeal swallow is important as defects in this process have been implicated in the pathogenesis of spastic esophageal disorders in humans including LES achalasia.(26) Swallow-induced LES relaxation is a vagally-mediated process with nitric oxide acting as the dominant neurotransmitter. Coinnervations by peptidergic capsaicin sensitive neurons are thought to play a modulatory role in maintaining LES tone with substance $\mathrm{P}$ antagonism causing a decrease in basal LES tone in a canine model. $(26,27)$

\section{Airway Protective Mechanisms}

Swallowing and respiration require shared use of multiple structures. For example, the pharyngeal musculature must dilate to allow airflow during breathing while also facilitating bolus conduction to the upper esophageal sphincter during pharyngeal 
swallow. As such, critical protective mechanisms must exist to prevent aspiration. (Figure 1) These include respiration/swallow coordination, i.e. "gate keeping," in addition to basal and response mechanisms. $(23,28)$

\section{"Gate keeping"}

Gate-keeping refers to the centrally-mediated coordination of swallow and respiration. In healthy people, breathing is a regular stereotyped behavior that may be interrupted by conscious ingestion of food or liquid, or unconsciously by swallowing of secretions.(29) Swallowing and breathing are under the regulation of central pattern generators located in the brainstem: the swallow central pattern generator (swCPG) and respiratory central pattern generator ( $\mathrm{rCPG})$, respectively. Stimulation of the swCPG results in sequential contraction of swallow effector muscles. Cross communications between the swCPG and rCPG coordinates swallowing and breathing such that swallowing occurs preferentially during specific phases of the respiratory cycle, and corresponds with the generation of swallow-apnea, a "swallow breath" which is a breath just prior to the onset of swallow, and respiratory phase resetting. $(23,29)$

Swallowing preferentially occurs during specific phases of the respiratory cycle. Timing of swallow varies with species. In humans, swallows are initiated during the postinspiratory and expiratory phases even if the swallow stimulus was delivered during inspiration. $(23,30)$ In both anesthetized and in awake, spontaneously swallowing dogs, nearly $80 \%$ of swallows occurred during the inspiratory phase.(31) Interestingly, respiratory swallow coordination may be impacted by posture. When humans adopt a feeding position more like dogs, swallowing occurs more frequently in the inspiratory phases in the breathing cycle.(32) Increases in inspiratory phase swallowing have been 
documented in the elderly and in patients with dysphagia. Both populations are at increased risk of aspiration.(33) Inspiratory phase swallowing has also been documented in humans with chronic respiratory disease, linking disordered respiration and swallow coordination with increased risk of AARS. $(23,29,34)$

Another means of airway protection during swallowing is swallow-apnea.(23) Studies in humans, as well as small and large animal models have documented a drop in airflow nearly to zero corresponding with pharyngeal swallow. This occurs as a result of powerful laryngeal adduction and restriction of diaphragmatic contraction. $(23,35,36)$ It should be noted that despite airflow decreasing to zero, some small-amplitude discharges are observed in electromyograms and neurograms of the diaphragm and phrenic nerve respectively.(23) It has been hypothesized that a "swallow breath" immediately before a breath-hold maintains a positive subglottic pressure permitting expiratory airflow after a swallow and clearance of residual secretions from the airway.(23) Multiple swallows in series prolongs swallow apnea. This places increased stress on patients with respiratory disease and is associated with disordered coordination of respiration and swallow. This may increase risk of aspiration in patients with existing respiratory disease contributing to disease exacerbations.(34) The disruption of the normal respiratory phase to accommodate a swallow causes the introduction of a new respiratory rhythm. Following apnea, the next breath is brought forward or delayed depending on the phase of the respiratory cycle when the swallow was initiated. $(37,38)$ Therefore, swallow affects not only the breath just following the swallow but all subsequent breaths as well.

\section{$\underline{\text { Basal mechanisms }}$}

Basal mechanisms are barriers to aspiration which are present independent of 
stimulation. The specific mechanisms encountered depend on the source of aspiration (i.e., gastroesophageal or oropharyngeal). In GER, gastric contents must span the length of the esophagus to reach the pharynx. In doing so, the refluxate must cross 2 highpressure zones: the LES and UES. The LES is a high-pressure zone at the distal esophagus comprised of intrinsic and extrinsic components. The intrinsic component consists of circular smooth or skeletal muscle fibers, depending on species. In humans and dogs this is a band of smooth muscle at the junction between the distal esophagus. $(39,40)$ The extrinsic component consists of external pressure from the diaphragm. These combined sources of pressure act as a barrier to reflux and must be overcome to allow bolus passage into the stomach from the esophagus. Basal pressure of the LES in non-anesthetized dogs is approximately $30 \mathrm{~mm} \mathrm{Hg}$ which, like in humans, has been demonstrated to be variable with body position and is reduced in patients under anesthesia. $(41,42)$ The UES serves as a barrier between the proximal esophagus and the pharynx. The role of the UES is to accommodate an anterograde swallowed bolus while preventing retrograde esophago-pharyngeal reflux and aspiration. The primary muscle responsible for closure of the UES is the cricopharyngeus (CP) muscle. The $\mathrm{CP}$ has several distinct characteristics compared to other striated muscles including increased density elastic connective tissue and sarcolemma. Because of these structural features, the UES maintains basal tone without active contraction. This tension increases with the degree of distention and allows a greater closing force to be applied behind a swallowed bolus.(43) The esophagus itself also has the capacity to contain refluxate until it can be cleared without allowing it to escape to the pharynx. Upon reaching the pharynx both GER and oropharyngeal aspiration encounter the same barriers to aspiration. The 
pharynx, like the esophagus, can contain certain volumes before it overflows into the airways. Within the airways and lungs, mucociliary clearance and innate immunity represent additional basal barriers to AARS by mitigating the impact of aspirated material on the respiratory tract. $(28,29)$ The immune response to aspiration will be discussed later in this chapter.

\section{$\underline{\text { Response mechanisms }}$}

Like the basal mechanisms, response mechanisms involve the pharynx, larynx, glottis, UES, esophageal body, LES, and airways. Unlike basal mechanisms, response mechanisms become active in response to mechanical or chemical stimulation. These can be subcategorized into 4 categories: (1) reflexes that clear volume from the pharynx and esophagus, (2) reflexes that accentuate the upper esophageal pressure barrier, (3) reflexes that induce closure of the larynx, and (4) clearing reflexes that remove aspirated material from the airways, i.e., cough and throat clear.

Reflexes that clear volume from the pharynx and esophagus include the reflexive pharyngeal swallow (RPS) and secondary peristalsis. $(44,45)$ The RPS acts as a major airway protective behavior by removing debris and residue from the entrance to the respiratory tract.(44) These responses have been demonstrated in large and small animal models by stimulation of the pharyngeal wall, epiglottis, larynx, or by injection of water into the pharynx in humans. $(24,44,46,47)$ This response is presumed to protect against spillage of oral contents into the pharynx during the oral/preparatory phase of swallowing, or in response to refluxate from the stomach. Unlike primary swallows, the RPS is not preceded by an oral/preparatory phase and lacks lingual peristalsis.(44) Otherwise RPS are indistinguishable from primary swallows. It has been documented that 
a larger volume is needed to trigger RPS in the elderly and that the rate of spontaneous swallow is lower which may predispose them to aspiration.(48) These swallows also play a significant role in airway protection during sleep in cases of nocturnal reflux.

Secondary peristalsis refers to peristalsis activated by distention. These occur in response to retained volume within the esophagus following primary peristalsis or in response to refluxed materials from the stomach. Distention activates a vagally-mediated reflex that leads to sequential activation of the esophageal musculature, relaxation of the LES and variable changes in UES pressure. Unlike primary peristalsis, secondary peristalsis is not preceded by a pharyngeal swallow. In this way, secondary peristalsis is similar to RPS.(45)

Reflexes that increase the upper esophageal pressure barrier include the esophagoUES contractile reflex, the pharyngo-UES contractile reflex, and the laryngo-UES contractile reflex.(49,50) The UES maintains a high-pressure zone between the esophagus and pharynx that relaxes in response to a pharyngeal swallow. However, the magnitude of pressure exerted by the UES is variable in response to level of arousal, degree of esophageal distention, location and rate of distention (slow vs. rapid, proximal vs distal esophagus), the type of distending material (gas vs. liquid, acidic vs. non-acidic), and body orientation.(49-52) Upper esophageal sphincter reflexes are mediated by complex circuitry that depends on the type, number and location of mucosal, submucosal and muscular receptors. In response to slow esophageal dilation, the UES responds by increasing tone.(51) This reflex is most pronounced by distention near the LES and is augmented by esophageal peristalsis to clear esophageal volume. Rapid esophageal gas distention however, results in UES relaxation. $(49,50)$ These responses are also 
influenced by body position and arousal. In humans, the upright position is associated with UES relaxation, rapid proximal esophageal distention, and is commonly associated with gas (eructation). UES contraction however, is more common during recumbancy, slow distal esophageal distention, and is associated with liquid refluxate.(53) This reflex also varies with arousal and is nearly abolished during deep sleep.(52) Studies investigating esophageal reflex responses in cats have demonstrated that the UES contractile response is mediated by vagal afferent fibers interacting with activated mucosal and mechanoreceptors.(54)

The pharyngo-UES contractile reflex has been documented with mechanical pharyngeal stimulation in cats (54) and water stimulation in humans, whereby water is injected into the pharynx to induce a response in the UES.(48) In both cases, stimulation resulted in increased UES tone. It is presumed that activation of this reflex prevents further leakage of gastric contents into the pharynx. This reflex response is nearly always followed by RPS. Topical anesthetics applied to the pharyngeal mucosa ablates this reflex.(55) The laryngo-UES contractile reflex describes increases in basal UES tone in response to laryngeal stimulation. While peripheral and central pathways are different between the pharyngo- and laryngo- UES contractile reflexes, both are thought to act to prevent additional GER from escaping into the pharynx. Interestingly, paradoxical and absent UES responses have been documented in people with UES dysphagia and those with reflux-induced laryngeal and pulmonary disorders demonstrating how reflux may perpetuate disease by diminishing airway protective mechanisms.(56)

Reflexes that induce laryngeal closure include the esophago-laryngeal closure reflex, pharyngo-laryngeal adduction reflex, and laryngeal adductor reflex. The 
esophago-laryngeal closure reflex occurs in response to esophageal distention. Abrupt distention of the esophagus is seen in response to reflux, vomiting and regurgitation. Studies in both animal models and people have documented reflexive adduction of the larynx in response to abrupt esophageal distention as a protective mechanism against aspiration.(57, 58) Unlike the esophago-UES reflex, abrupt esophageal distention triggers laryngeal closure regardless of location. Esophageal stretch receptors in the esophageal body and vagal motor fibers have been implicated as the afferent and efferent arms of this response respectively.(58) The role of this response is presumed to be a protective mechanism in response to belching, reflux, regurgitation and vomiting.(59) Experimental feline models have demonstrated that esophagitis significantly reduces the strength and frequency of this response.(55) Additionally, treatment with topical lidocaine or capsaicin, or removal of the esophageal mucosa significantly inhibits this protective reflex. This suggests that mucosal stimulation plays a significant role in the induction of this reflex.(60)

The pharyngo-laryngeal adduction reflex refers to the brief closure of the larynx in response to pharyngeal stimulation. $(61,62)$ This reflex has been stimulated with experimental injection of water into the pharynx and in dysphagic patients with demonstrated overflow of ingesta into the pharynx during the oral/preparatory phase. The adduction of the larynx is believed to reduce the risk of significant aspiration by decreasing the diameter of the glottal opening of the larynx during swallowing. This response is absent in dysphagic patients with predeglutitive aspiration (i.e. aspiration prior to pharyngeal swallow), supporting the role of this reflex as an airway protective 
behavior.(63)

The laryngeal adductor reflex occurs in response to stimulation of the larynx mediated by the cranial laryngeal nerve. The reflex is activated by mechanical, chemical, or thermal stimuli. Importantly small animal experimental models and human studies have demonstrated suppression of this response with increased anesthetic depth suggesting that diminished laryngeal reflexes may result in increased risk of aspiration during sedation and anesthesia and that it may be possible to modulate the degree of impact on airway protective mechanisms by careful selection of anesthetic protocols .(64)

The most easily recognized and evaluated airway protective mechanisms are cough and throat clear (TC). Cough and TC play an important protective role that facilitates clearance of particulate matter, irritants, debris, and/or fluid from the respiratory tract.(65) Cough, when excessive, may also become harmful to the airways and negatively impact the quality of life for both dog and client.(65-67) This highlights the need to balance targeted therapy allowing adequate airway protection while not allowing cough to become detrimental. Cough can be subdivided into 3 phases: an inhalation phase, a pressurization phase, and an expulsion phase that forcefully ejects air against a closed glottis. A TC lacks an inhalation phase but otherwise serves a similar role. Both cough and TC are stimulated by chemical or mechanical stimuli. $(68,69)$ Cough is triggered by stimulation of the pharynx, larynx, trachea, carina, and bronchi while TC is typically associated with stimulation of the pharynx, larynx, and cervical trachea only. $(67,69)$

Induction of cough in humans and animal models, including dogs, is thought to arise from a dual sensory system. C-fibers are mechanically insensitive but respond to a 
wide variety of chemical mediators including prostanoids, bradykinin, and ions. These also contain tachykinin-expressing neurons including transient receptor potential vanilloid 1 (TRPV1) and transient receptor potential ankyrin 1 (TRPA1).(1, 70-72) The second sensory arm of cough involves mechanically sensitive myelinated vagal afferent fibers that are localized to the trachea, and mainstem and segmental bronchi.(73) Though these are largely considered chemically insensitive due to the absence of TRPV1 and TRPA1 ion channels, they are activated by protons by another unknown acid sensing ion channel. These receptors are responsive to touch-like mechanical stimulation. Peripheral terminals are confined to the space between the epithelial cell layers and smooth muscle.(1) Pulmonary stretch receptors are critical in determining lung volume but they are not thought to participate directly in cough beyond modulating sensitivity to tussive stimuli and determining pre-cough inspiratory volumes.(74)

Central mechanisms regulating cough include synergistic reflex bronchospasm as a result of vagal afferents converging in the caudal brainstem.(75) Further C-fiber activation has been demonstrated to have a sensitizing effect on cough receptors via a central nervous system dependent mechanism, echoing central pain sensitization in somatic tissues.(76) In people, cough can also be generated from extra-pulmonary structures including the ear, nose, pharynx, and esophagus.(1) While extrapulmonary cough has been presumed in dogs, the same degree of characterization has not been performed compared to other AARS.(12, 77-79) Clinical recognition of extra-pulmonary sources of cough is particularly important as disease may be missed by routine thoracic imaging.(80) The source of cough in these regions is theorized to be multifactorial with both direct stimulation of sensory afferents and secondary sensitization being implicated. 
Cough secondary to esophageal stimulation has been demonstrated both in people and in animal models. While aspiration of refluxate has been demonstrated, this is not necessary to generate cough. Shared vagal innervation between the esophagus and airways result in increased mucous secretion and bronchospasm which may generate cough without aspiration.(81)

\section{Aspiration and Respiratory Inflammation}

Aspiration has been implicated in extra-pulmonary (i.e., laryngeal, pharyngeal, middle ear, etc.), airway, and pulmonary parenchymal disorders through the action of acid, digestive enzymes, and aspiration of foreign material into the airways.(12, 28, 8284) As $50 \%$ of healthy people aspirate during sleep without clinical significance,(85) it is

likely that the development of disease depends on the content and volume of the aspirated material rather than the act of aspiration itself. Aspiration may be witnessed or occult and may be oropharyngeal or gastroesophageal in origin.(86)

In people, the inhalation of low $\mathrm{pH}$ gastric fluid, digestive enzymes, and/or particulate material frequently leads to sterile aspiration pneumonitis which may or may not develop a secondary bacterial complication (aspiration pneumonia).(86, 87) However, lack of clinically distinguishing features leads to over-diagnosis of secondary bacterial pneumonia and potentially inappropriate treatment. $(86,87)$ This distinction is rarely made in veterinary medicine and may contribute to antimicrobial overuse in veterinary patients. In people, large volume aspiration may lead to a severe form of acute respiratory distress syndrome (ARDS). In people with aspiration pneumonitis, one third of patients develop a severe prolonged disease course associated with ARDS. $(86,88,89)$ Alternatively, chronic aspiration of small volumes (micro-aspiration) may lead to diffuse 
aspiration bronchiolitis and has been speculated by some to lead to disease exacerbations in, if not the development of, idiopathic pulmonary fibrosis. (89)

Inflammation in acidic aspiration is frequently modeled by instillation of hydrochloric acid into the trachea of small animal models.(90) In rodent models, the response to acid instillation is characterized by a bi-phasic inflammatory response where an immediate response involving capsaicin sensitive TRPV-1 neurons and direct damage to the respiratory epithelium, is followed 4-6 hours later by IL-6 and IL-8 mediated neutrophilic inflammation. Subsequent extravasation of fluid and protein into the alveoli and airways due to loss of pulmonary vascular integrity increases work of breathing and airway resistance, decreases lung compliance, impedes diffusion of oxygen, and interferes with pulmonary surfactant. $(86,90-93)$ Decreasing airway and pulmonary $\mathrm{pH}$ has additional negative effects on normal immunologic responses. Antimicrobial peptide activity, cytokine expression, and macrophage activity have all been demonstrated to decrease in response to acidic conditions.(86)

Like acid, digestive enzymes play a role in respiratory inflammation following aspiration. Pepsinogen is produced in the chief cells of the gastric mucosa and undergoes conversion to pepsin in response to a low $\mathrm{pH}$. Deactivation begins at a $\mathrm{pH}$ of 6.8 before becoming irreversibly deactivated at $\mathrm{pH}$ 7.5. A human bronchial epithelial cell model found pepsin to be directly cytotoxic and induce an inflammatory response. An in vivo study in rabbits demonstrated erosive esophageal lesions secondary to pepsin exposure were caused by destruction of junctional molecules.(94) Similar lesions have been identified in airways following aspiration of gastric fluid. $(9,94)$ Importantly for the role of pepsin in the development of upper airway disease, pepsin sensitive receptors have 
been demonstrated in human laryngeal tissue. $(95,96)$ After binding, pepsin is taken up into endocytic vesicles where the $\mathrm{pH}$ is approximately 5 . At this $\mathrm{pH}$ pepsin that is not irreversibly denatured may become re-activated.(96) Pepsin at a pH of 5 has demonstrated $40 \%$ of its maximal proteolytic activity. $(86,97)$ Epithelial damage to endocytosed pepsin in the upper airways has been demonstrated in people and highlights the role of non-acidic, extra-esophageal reflux in the pathogenesis of upper airway inflammation. $(9,95,96)$ Bile acids (BA) have been documented in BALF fluid in human patients with reflux-associated respiratory disease and in dogs with pulmonary fibrosis.(98-100) Studies have implicated BA aspiration in pulmonary infections by Pseudomonas aeruginosa. BA aspiration is associated with increased expression of IL-8 and tumor necrosis factor alpha (TNFa) in animal models though the complete inflammatory mechanism is incompletely understood.(86)

Small non-acidified gastric particles (SNAPs) also induce inflammation in the respiratory tract. In small animal models, tracheal instillation of small $(<10 \mathrm{um})$ particles lead to neutrophilic inflammation at 4-6 hrs.(97) A lack of pulmonary edema distinguishes SNAP induced inflammation from neutrophilic inflammation secondary to aspiration of acidic gastric contents.(97) In small animal models, monocytic influx occurs after 48 hours initiating the formation of granulomatous inflammation by a monocyte chemoattractant protein-1 (MCP-1) mediated process.(97) Importantly, the combination of acidic aspiration and SNAP aspiration appears to have a synergistic effect. Albumin concentrations in BAL fluid, which serves as a marker of alveolar capillary membrane integrity, are significantly higher in animal models with combined acid and SNAP 
aspiration compared either acid or SNAP alone. $(86,97)$

\section{Risk factors for aspiration}

Increased risk for developing AARS have been reported for human patients with altered consciousness, impaired airway protective mechanisms, and conditions capable of overwhelming barriers to aspiration.(97) These risk factors are mirrored in dogs where level of consciousness, body position during anesthetic recovery, duration of anesthesia, vomiting and regurgitation, seizures, cranial nerve deficits and the presence of megaesophagus are independent risk factors for developing aspiration pneumonia following surgery.(101, 102) More than 1 disorder may occur concurrently with dogs having 1, 2 and 3 associated disorders in $68 \%, 26 \%$, and $6 \%$ of cases respectively.(102) The most common underlying disorders included esophageal disease (39.8\%), vomiting (38.6\%), neurologic disease (27.3\%), laryngeal disease (18.2\%), and post-anesthetic aspiration (13.6\%).(102) Of those with esophageal disease, megaesophagus (ME) was identified in $71.4 \%$ of dogs. The remaining dogs were diagnosed with non-ME esophageal dysmotility, hiatal hernia, and an unknow disorder in $17.1 \%, 2.8 \%$, and $8.6 \%$ of dogs respectively.(102) Though focusing specifically on aspiration pneumonia, these studies highlight the role of alimentary disease, particularly esophageal disease, in the development of aspiration. Given the breadth of aspiration syndromes, the role of alimentary dysfunction in the development of respiratory disease may be even greater than these studies suggest and warrants further study.

\section{Recognized Aspiration Related Respiratory Syndromes in Dogs}

The ultimate prevalence of aerodigestive disease in dogs is currently unknown, however they represent a significant source of morbidity and mortality in human patients 
and are associated with disease progression, exacerbations of clinical signs, and treatment costs.(103) As dogs are susceptible to many of the same conditions as people a similar spectrum of disorders should be expected with number of similar conditions being reported in individual case reports and case series.(12, 79, 104-106) An extensive review has been performed previously and is beyond the scope of this manuscript,(12) however for a brief overview aspiration associated respiratory syndromes reported in dogs and in people are presented in Table 1.

\section{Diagnostic Testing for Aerodigestive Disorders}

Thoracic radiographs are often considered the first line diagnostic for patients presenting evidence of respiratory disease. Though widely available, radiographs are poorly sensitive for aerodigestive disease where signs are elicited by stimulation of the esophageal-bronchial reflexes as well as mechanical/chemical stimulation of the pharynx and larynx which are poorly imaged by standard thoracic radiography.(107-111)

Adjunctive diagnostic and monitoring strategies for GER and EER in veterinary medicine incorporate a combination of client surveys, treatment trials with proton pump inhibitors (PPIs), esophagoscopy, and rarely esophageal manometry and ambulatory $\mathrm{pH}$ probes.(19, 112-119) However, these have significant limitations. Client reporting is inherently prone to bias due to variable client vigilance, and a failure to recognize episodic, subtle clinical signs. Treatment trials with antacid therapy may be associated with a large and variable placebo effect. In people, randomized control trials that included a placebo arm have documented improvement in $1 \%$ to $40 \%$ in the placebo group, often meeting statistical significance.(120, 121) Diagnostic tests relying upon esophageal or pharyngeal $\mathrm{pH}$ fail to recognize reflux in human patients treated with PPIs or those with 
non-acidic reflux, which is being increasingly implicated in disease representing up to $90 \%$ cases in some human studies. $(9,122-124)$ Diagnostics requiring anesthesia impart increased risk of aspiration events due to decreased airway protective mechanisms. In human studies endoscopy identified abnormalities in less than $50 \%$ of patients with known disease.(116) High resolution manometry and $\mathrm{pH}$ probes are significantly limited by availability, cost, and the need for substantial operator training. $(18,19)$ As such, additional diagnostic and monitoring strategies are needed.

The current methods of evaluating aerodigestive disease in veterinary patients are limited necessitating investigation into novel strategies. Several avenues of potential investigation exist both for diagnosing aerodigestive disease and for objective disease monitoring. These include videofluoroscopic swallow studies (VFSS), reflux scintigraphy, biomarker development, and acoustic wave analysis.

\section{$\underline{\text { Videofluoroscopic swallow studies }}$}

Videofluoroscopic swallow studies (VFSS) are considered the criterion standard for the evaluation of dysphagia in dogs.(125) Historically, these have been performed with dogs held in lateral recumbancy and force-fed. This has limited its use in several disorders due to unacceptable risks of aspiration. Recent studies have proposed an alternative which, by allowing free-feeding, reduces risk of aspiration to what would be expected from feeding at home. Natural feeding position and standardized food items with rheological properties objectively consistent with commercially available products, further increases the physiologic relevance of VFSS in dogs. (125) Utilizing this technique for evaluation of aerodigestive disorders in dogs may provide valuable information for dogs with occult aerodigestive disease, or help identify patients at risk for 
developing an ARRS. Chapter 2 describes our investigation of VFSS in dogs with cough lacking alimentary tract signs. Chapters 3-4 detail investigations of a novel cause for canine megaesophagus and associated directed therapy.

\section{$\underline{\text { Scintigraphy }}$}

Nuclear scintigraphy, which has been employed to measure mucociliary clearance in dogs,(126) is also capable of measuring reflux and cumulative small volume aspiration.(127-129) It does not rely on $\mathrm{pH}$, is non-invasive, and can be performed without anesthesia.(127-131) This technique is considered safe and has been used to evaluate pulmonary aspiration in fragile human infants.(127, 130) In adults, cough and laryngospasm were strongly correlated with positive reflux/aspiration scintigraphy.(130) Further, patients who were positive for reflux on scintigraphy had symptomatic response to surgical treatment. (130) Adaptation of this technique in dogs may allow detection of microaspiration, which may not be detectable by conventional imaging modalities. Before placing pathologic significance on findings in clinically affected dogs, normative data is needed. Chapter 5 describes our use of reflux scintigraphy to evaluate for GER, EER, and aspiration in heathy dogs.

\section{$\underline{\text { Biomarkers of Reflux }}$}

Biomarkers are used to diagnose, prognosticate, and identify populations at risk for disease. In recent years biomarkers had a progressively larger impact in companion animal medicine.(132) Biomarkers have the potential to advance our understanding of disease pathogenesis and allow objective investigation of efficacy of novel therapeutics in various disease states. Biomarkers have been used reliably to provide evidence of reflux and aspiration in human patients. $(100,122,133)$ Though not used routinely in veterinary 
medicine, there is evidence that aspiration may be detected in respiratory secretions in veterinary patients.(98) Further evaluation of potential biomarkers is warranted to detect patients with reflux associated respiratory syndromes. Chapter 6 describes our study characterizing the gastric fluid proteome of dogs as well as the oropharyngeal proteome in healthy, coughing, and vomiting or regurgitating dogs. This pilot discovery study is indented to evaluate for potential protein biomarkers of reflux and aspiration.

\section{Acoustic cough monitoring}

Cough represents both a critical defense mechanism for the respiratory tract as well as a marker for disease control. Cough additionally represents a significant source of frustration for both patient and client. Unfortunately, management of cough in veterinary medicine is often suboptimal and based on subjective response rather than objective evidence. Acoustic wave analysis incorporating spectral and waveform evaluation has provided an objective means of detecting cough in people but has not yet been adapted for veterinary use.(134-139) This may provide a means of objectively evaluating cough frequency in veterinary patients and bridge the gap between subjective monitoring and objective assessment. Chapter 7 describes our study characterizing the canine cough acoustic wave form and comparison to other acoustic behaviors to determine its potential utility as an objective disease marker in dogs with cough.

\section{Conclusions}

Aerodigestive diseases are the result of discordant respiration and swallow and are currently underdiagnosed in our canine patients. The close inter-relationship between these two antagonistic processes represents a broad range of diagnostic and clinical challenges. Further investigation into these conditions in dogs requires advancing 
respiratory diagnostics beyond thoracic radiography to include dynamic and functional imaging as well as objective monitoring strategies to identify a potentially underrecognized patient population. Identification and characterization of patients with aerodigestive disease may further opens the doors for the development of novel targeted interventions. The following chapters detail our contributions to this endeavor. 


\section{CHAPTER 2}

\section{AERODIGESTIVE DISORDERS IN DOGS EVALUATED FOR COUGH USING RESPIRATORY FLUOROSCOPY AND VIDEOFLUOROSCOPY SWALLOW STUDIES}

\section{Introduction}

Aerodigestive disorders in people represent a broad spectrum of diseases that emphasize the complex interrelationship between respiration and swallowing. $(140,141)$ Unfortunately, diagnosis is challenging as these conditions frequently present without gastrointestinal signs.(142) Aerodigestive disease(s) (AeroD) are infrequently investigated in dogs due a combination of poor clinical recognition and limitations in available diagnostics. Aspiration pneumonia (AP) is the most well recognized example of AeroD in dogs. However, in humans and less commonly dogs, macro-aspiration and chronic micro-aspiration have also been associated with upper and lower airway dysfunction, bronchiolar diseases, interstitial lung disease, and aspiration pneumonitis.(5, $14,143,144)$ Reflux is a common source of chronic microaspiration in people, resulting in acute and chronic pulmonary disease. The prevalence of reflux in chronic cough, asthma and chronic obstructive pulmonary disease (COPD) is $50 \% .(2,5,13,14)$ Comparable studies in dogs with respiratory symptoms are lacking.

Thoracic radiographs are often considered a first line diagnostic test for dogs with respiratory disease. Though widely available, radiography is insensitive compared to alternative imaging modalities for a number of respiratory syndromes.(109-111) This may be especially true for those with AeroD, where cough may be elicited by stimulation of the esophageal-bronchial reflex. This reflex, which involves vagally-mediated 
bronchoconstriction secondary to acidic stimulation of the distal esophagus, underscores the interplay of acid reflux with respiratory clinical signs. Mechano- and/or chemoreceptor stimulation of the pharynx, larynx, and cervical trachea can also trigger cough which is not well evaluated by standard thoracic radiography.(107, 108) As such, alternative diagnostics are frequently required. $(145,146)$

The association between reflux, aspiration, and airway dysfunction has not been thoroughly evaluated in veterinary medicine, though the link has been supported by a number of clinical case reports and experimental canine models.(15, 17, 79, 144) Identifying patients affected by AeroD could prove important as uncontrolled reflux in people leads to disease progression, exacerbations of clinical signs, increased patient morbidity, and treatment costs.(11) Patients with a history of cough which worsens during eating and drinking, or those with concurrent regurgitation/vomiting may prompt consideration of AeroD. However, a subpopulation with occult alimentary disease may prove clinically important by identifying new therapeutic targets and/or by providing a diagnosis in dogs with persistent cough despite unremarkable conventional diagnostics (i.e., idiopathic cough).

Videofluoroscopic swallow studies (VFSS) are the criterion standard for evaluating dysphagia in dogs.(147) It is unknown if VFSS could identify subclinical pathology in dogs without dysphagia or vomiting, but with cough. The objective of this study was to evaluate dogs presenting exclusively for cough using respiratory fluoroscopy (RF) and VFSS to detect occult AeroD. We hypothesized that a subset of dogs presenting exclusively for cough would have documentable evidence of AeroD despite the absence of esophageal or gastrointestinal signs. 


\section{Materials and Methods}

\section{$\underline{\text { Case Selection and Criteria }}$}

Medical records for dogs presenting to the University of Missouri Veterinary Health Center (MU-VHC) between April 2015-December 2017 with RF and VFSS were retrospectively reviewed. Dogs were included if they had a primary complaint of cough without esophageal and gastrointestinal signs, had thoracic radiographs and complete medical records. When thoracic radiographs were performed with a referring veterinarian and unavailable for review, a radiology report from a boarded radiologist was considered adequate for a final radiographic diagnosis. Our exclusion criteria were dogs with cough of cardiac origin or esophageal/gastrointestinal signs including dysphagia, regurgitation, and vomiting within the preceding 6 months. A terminal retch, after a paroxysm of cough, was not considered evidence of gastrointestinal disease and therefore did not meet our exclusion criteria.

\section{Data extracted from the medical record}

Demographic data, body weight (BW) (kg), body condition score (BCS), headconformation, other clinical signs aside from cough, duration of cough, radiographic diagnosis, and final clinical diagnosis were acquired from the medical record. The results from laryngeal function examination, bronchoscopy, bronchoalveolar lavage fluid (BALF) cytology, and culture results were reported when available.

\section{$\underline{\text { Respiratory fluoroscopy and videofluoroscopic swallow study }}$}

Dogs meeting our inclusion criteria had their RF and VFSS evaluated by two internal medicine specialists (MG, CR) and a board-certified radiologist (IM) for standardized outcome parameters. (Table 2) Criteria for evaluation are displayed in 
Tables 3 and 4. Studies were performed at 30 frames/sec using a GE Advantx or GE OEC 9900 Elite Mobile C-Arm system (GE Heathcare, Chicago, IL) at the MU-VHC.

Respiratory fluoroscopy preceded the VFSS. The VFSS was performed as previously described.(147, 148) Briefly, after a 12-24 hour fast dogs were placed in a polycarbonate kennel appropriate for their body size: small/toy ( $\leq 35 \mathrm{lbs}$ ), medium (>35 lbs $\leq 65 \mathrm{lbs})$, large ( $>65 \mathrm{lbs}$ to $\leq 85 \mathrm{lbs}$ ), and giant breed dogs ( $\geq 85 \mathrm{lbs}$ ). Dogs were fed three standardized food consistencies containing a contrast agent: puree (25\% iohexol (350 $\mathrm{mg} / \mathrm{mL})$ ), liquid (25\% iohexol (350 mg/mL)), and kibble (barium $40 \% \mathrm{w} / \mathrm{v}$ ).

\section{$\underline{\text { Final Diagnosis }}$}

Canine chronic bronchitis (CCB), eosinophilic bronchitis (EB), AP, laryngeal paresis/paralysis, epiglottic retroversion (ER), bronchomalacia (BM), bronchiectasis (BE), and hypoplastic trachea (HT) were diagnosed as previously described.(149-155) Specific diagnostic criteria are available in Table 5. Non-respiratory or "alimentary" cough was diagnosed where there was a VFSS abnormality supportive of an AeroD in the absence of an inflammatory or architectural respiratory disorder based on the diagnostics performed.

\section{$\underline{\text { Statistics }}$}

Descriptive statistics were performed where appropriate. A Wilcoxon rank-sum test or a one-way ANOVA on ranks was performed to detect significant differences in RF and VFSS parameters for demographic data, BW ( $\mathrm{kg})$, BCS and head-conformation. A $p$ $<0.05$ was considered significant. Post-hoc analysis (Dunn's method for multiple comparisons) was performed where appropriate.

\section{Results}




\section{$\underline{\text { Animals }}$}

One hundred and thirty VFSS were performed between April 2015 and December 2017 with 31 dogs meeting criteria for further evaluation. Demographic and clinical data are presented in Table 6.

\section{Diagnostic Evaluation}

Multiple ancillary respiratory diagnostics were performed. With each test, some dogs had more than one abnormality identified. Results are presented in Table 6 .

With respect to VFSS, abnormalities were detected for 25/31 (81\%) dogs, including 9/11 (82\%) dogs with unremarkable radiographs. Six of $11(55 \%)$ dogs with a bronchial pattern on radiographs and 6/9 (67\%) dogs with sterile neutrophilic and/or eosinophilic inflammation on BALF had VFSS abnormalities. All dogs with BE ( $\mathrm{n}=5)$ and BM $(\mathrm{n}=5)$ had VFSS abnormalities. Five of $7(71 \%)$ dogs with radiographic evidence of AP had abnormalities detectable on VFSS, including all of those with a history of recurrent AP.

Oral-preparatory phase defects were found in $6 / 25$ dogs. Penetration was observed in 5 and aspiration in 3 dogs with both penetration and aspiration in 2 of those dogs. (Figure 3) Penetration of the larynx was exclusively by kibble. All aspiration events occurred with puree and/or liquid. Both were commonly in conjunction with pharyngeal dysfunction (pharyngeal hypomotility (PH), $n=4$; pharyngeal spasticity, (PS) $n=1$ ). Pathologic reflux was noted in $36 \%$ of dogs with abnormal VFSS.

Epiglottic retroversion was identified in 2 dogs by RF; only 1 of these dogs was also identified by functional oral/laryngeal function examination. A laryngeal polyp was identified in one dog via RF and was associated with aspiration and pathologic GER. In 
6/7 dogs (85.7\%) with LarPar, an abnormal VFSS was present. Abnormalities included PH (3/7), GER (3/7), penetration + aspiration (2/7), and EH (1/7). No significant differences were found for any VFSS based on demographic data, BW $(\mathrm{kg})$, BCS, or head-conformation. A summary of all VFSS findings are presented in Table 7.

\section{$\underline{\text { Final clinical diagnoses }}$}

Diagnosis of more than one disease was frequently made in dogs with either respiratory cough or non-respiratory alimentary cough. (Table 7) Respiratory cough without VFSS evidence of alimentary disease was found in 4/31 (13\%) dogs. Respiratory cough with concurrent VFSS abnormalities was found in 17/31 (55\%) dogs. Alimentary cough was identified in 8/31 (26\%) dogs. Neither a respiratory nor alimentary source of cough could be identified in 2 dogs.

\section{Discussion}

In dogs presenting for cough without clinical evidence of alimentary disease, VFSS documented abnormalities in $81 \%$ of cases highlighting the common link between the respiratory and alimentary tracts (i.e., AeroD). Identifying dogs with disorders of the pharynx, esophagus and stomach as a primary source of or contributor to their respiratory signs may enhance our understanding of the pathogenesis of chronic respiratory disease in dogs, opening the door for evaluation of novel, targeted therapies.

Aerodigestive disorders span a broad range of diseases reflecting defects in respiratory-swallow coordination. These represent a diagnostic challenge because many patients present without gastrointestinal signs. Importantly, GER occurs commonly in healthy, asymptomatic humans and physiologic reflux been documented in up to $41 \%$ of asymptomatic dogs using VFSS.(147) Pathologic and physiologic reflux differ in volume, 
timing and location within the esophagus. In people, pathologic reflux often remains occult until patients develop deleterious sequelae: esophagitis, laryngeal dysfunction, regurgitation and a wide spectrum of respiratory diseases. The association between reflux, aspiration, and airway dysfunction has not been thoroughly evaluated in dogs, despite several supportive clinical case reports, experimental canine models, and a review of aspiration-related respiratory disorders. $(15,17,79,144,149)$ Detection of reflux and aspiration is possible by several imaging modalities including VFSS.

Videofluoroscopic swallow studies are sensitive for identifying human patients with aspiration secondary to dysphagia.(156) Historically, the use of VFSS for dogs with esophageal dysphagia (e.g., ME), a risk factor for aspiration, has been limited. This is largely due to the historical VFSS protocol of restraint in lateral recumbancy and forcefeeding which carry unacceptable risks of aspiration. $(157,158)$ By allowing free-feeding in unrestrained dogs, the risk of AP is no more than would be expected when feeding at home and allows evaluation of patients for which a VFSS would have been previously contraindicated.(147) Additionally, the upright and free-feeding protocol allows for a more physiologic reflection of (often subclinical) alimentary defects that can contribute to a wide variety of respiratory diseases.

In our study, VFSS abnormalities were detected in $81 \%(25 / 31)$ of dogs presenting with cough with no owner-reported alimentary tract signs. This demonstrates that, like people, AeroD presents in the absence of dysphagia, regurgitation or vomiting. This study emphasizes a previously under-recognized and common canine population with alimentary tract disease causing or contributing to cough. Approximately $26 \%$ of dogs in this study were diagnosed with alimentary or "idiopathic cough" based on their 
diagnostic evaluation. Respiratory cough with VFSS abnormalities was found in 17/31 (55\%) of dogs, reflecting the complex relationship between respiratory and alimentary disease; it is likely that each contributes to disease progression.(159) For example, reflux contributes to laryngeal dysfunction, a known risk factor for aspiration. $(79,160)$ Likewise, cough induces reflux in humans likely contributing to a self-perpetuating cycle in some patients.(161) Therefore, treatment in dogs with mixed respiratory and alimentary disease is likely to be multimodal reflecting the contribution of alimentary tract disease in such cases. Given the percentage of dogs with alimentary or mixed alimentary and respiratory disease, AeroD should be investigated in dogs with chronic cough and not solely in those with evidence of AP. Identifying dogs with AeroD is important as failing to treat occult GI disease may, as in people, allow for progression of disease and contribute to patient morbidity and mortality. $(5,108,117,140,162,163)$ Based on our study, evaluation of alimentary tract disorders using VFSS should be considered as a part of the clinical evaluation of coughing dogs.

Supportive respiratory and/or neurologic signs may further increase our index of suspicion for AeroD. In our study, all dogs with historical recurrent AP had VFSS abnormalities. Such historical information may increase the index of suspicion for AeroD in patients. Additionally, 6/31 (19\%) of dogs had a history of worsening clinical signs during eating and drinking. As such, specific lines of questioning emphasizing the relationship between the respiratory and alimentary tracts may be helpful in identifying patients with AeroD. Concurrent nasal disease (sneezing, nasal discharge and reverse sneezing) were also encountered, paralleling human studies where extra-esophageal reflux (EER) results in nasopharyngeal as signs as well as cough. $(4,117,164)$ Four dogs 
had neurologic deficits on physical examination. Though this was identified in a relatively small number of patients, this study and others support a relationship between neurologic dysfunction, dysphagia, and respiratory disease. $(149,165)$ As such, studies evaluating patients with neurologic dysfunction for occult AeroD may be warranted.

Thoracic radiographs are considered a first line diagnostic in patients with chronic cough. $(145,146)$ However, thoracic radiographs fail to identify the source of disease especially when it is dynamic (intermittent or changes are dependent on phase of respiration), extra-thoracic in origin, subacute to acute where radiographic lesions lag behind clinical signs, or with certain small or subtle lesions. $(145,146,166)$ In this study, $35 \%$ of dogs had unremarkable radiographs despite nearly $82 \%$ having detectable abnormalities on VFSS. This speaks to a lack of sensitivity for detecting the source of AeroD by radiographs alone and underscores the utility of VFSS as an adjunctive tool in dogs with cough, particularly in the face of unremarkable thoracic radiographs.

A bronchial pattern on radiographs, which was found in $11 \mathrm{dogs}$, is considered non-specific evidence of lower airway disease.(111) In isolation, this finding provides little information to aid in clinical decision making without additional diagnostics including BALF cytology/culture. In our study 7/11 patients with a bronchial pattern were later diagnosed with inflammatory airway disease based on BALF cytology. Six of those 7 dogs had abnormalities detectable on VFSS. Investigating a link between chronic inflammatory disease and repetitive microaspiration is warranted. Though markedly under diagnosed by thoracic radiographs, $\mathrm{BE}$ and $\mathrm{BM}$ were each detected in 5 dogs in this study. Microaspiration has been implicated in the pathogenesis of sterile airway inflammation in people,(167) and is a known contributor to the development of BE and 
BM.(168-170) Four of 5 cases of BE and BM were associated with VFSS abnormalities including laryngeal penetration, reflux, and pharyngeal/esophageal dysmotility suggesting that, like people, AeroD may contribute to airway inflammation and architectural remodeling. Aspiration pneumonia is the most widely recognized AeroD in dogs. Radiographic evidence of AP was present in $23 \%$ of patients in our study including 2 dogs with a concurrent diffuse bronchial pattern. The majority (71\%) of these dogs had abnormalities on VFSS suggesting that dogs with chronic clinical signs ( $>2 \mathrm{mo})$ and radiographic evidence of AP may warrant a more thorough investigation than radiographs alone. As such, a thorough diagnostic evaluation of respiratory disease in veterinary medicine should be multimodal with clinical consideration for AeroD.

Laryngeal dysfunction was abnormal in 12 dogs despite only 3 presenting with dysphonia. Laryngeal erythema was found in $80 \%$ of dogs that underwent a laryngeal function examination including all dogs with evidence of pathologic reflux. While laryngeal erythema has been previously discussed as a non-specific finding of chronic cough,(105) based on the findings of this study, a contribution from EOR would be strongly suspected. Laryngeal paralysis, a known risk factor for AP, is not considered a consequence of chronic cough but has been associated with reflux diseases as well as other dysphagic disorders. $(149,160)$ These findings were supported by our study where 6/7 dogs with laryngeal dysfunction has concurrent swallow study abnormalities, including PH, penetration, and aspiration. Common innervations to pharynx, larynx, and proximal esophagus through the recurrent larnygeal nerve may explain this spectrum of dysfunction, and may suggest an increased risk of aspiration in these patients beyond laryngeal dysfunction alone.(165) Penetration or aspiration was witnessed in 6 dogs (8 
total incidences). Two dogs with documented aspiration exhibited no attempts to clear the aspirated material from the trachea (Penetration-aspiration score of 7). In both cases aspirated material extended past the thoracic inlet within the trachea suggesting diminished airway protective mechanisms. While evidence of aspiration during VFSS helps confirm the link between an abnormal swallowing and respiration, absence of witnessing aspiration during the limited period video clips are obtained does not rule it out. Patients with penetration of kibble without aspiration on VFSS were considered at high risk for future macro-aspiration events (171) and possibly microaspiration. Microaspiration is not detectable via VFSS.

Fluoroscopy has advantages over thoracic radiography in that VFSS capture data over a longer period and dynamic processes are captured. However, intermittent abnormalities may be missed using this modality. Thus, an important limitation of this study is the duration of data collection. Additionally, VFSS for detection of dogs with occult AeroD is not widely available requiring referral; not all referral centers use unrestrained free-feeding protocols. The retrospective nature of this study is another limitation wherein not all dogs received the same diagnostic evaluation. Future prospective studies are warranted.

\section{Conclusions}

Aerodigestive diseases occur in dogs in the absence of esophageal and gastrointestinal signs and in the face of normal thoracic radiographs. Identifying this under-recognized patient population opens doors for treatment targeting alimentary tract disease in dogs with what may have previously been considered "idiopathic cough." This study highlights the need for multimodal evaluation incorporating VFSS in dogs 
presenting with cough, regardless of presence of alimentary tract signs and particularly for those with unremarkable thoracic radiographs. 


\section{CHAPTER 3}

\section{VIDEOFLUOROSCOPIC SWALLOW STUDY CHARACTERIZATION OF LOWER ESOPHAGEAL SPHINCTER ACHALASIA SYNDROME (LES-AS) IN DOGS}

\section{Introduction}

Canine megaesophagus (ME) is a motility disorder of the esophagus carrying a poor long-term prognosis with death frequently reported secondary to aspiration pneumonia, malnutrition, and euthanasia due to poor quality of life.(172-174) Classically, ME is described as congenital or acquired and as idiopathic or secondary to a number of underlying etiologies including hypoadrenocortisolism and myasthenia gravis (MG).(175-177) However, identification of an underlying disease fails to reveal if a functional outflow obstruction of the esophagus is causing ME. This distinction is critically important in people where lower esophageal sphincter (LES) disorders causing functional obstruction, LES achalasia, are rare causes of ME that respond to targeted therapy designed to address esophago-gastric outflow obstruction.(178-180) This condition is considered distinct from conditions that cause esophageal hypomotility without functional LES obstruction. Achalasia, a primary esophageal motility disorder in people, results from a selective loss of inhibitory myenteric neurons leading to a failure of the LES to relax in response to pharyngeal swallow and impaired esophageal peristalsis.(181) While sporadic cases of functional LES obstruction have been suspected in dogs, comparable etiologic information is lacking. Functional LES disorders in dogs are rarely diagnosed due to limitations in currently available testing used in dysphagia evaluation.(182-185) However, functional LES disorders may represent an important, yet undetected, subpopulation of dogs with ME, perhaps responsive to targeted 
intervention and with a different long-term prognosis.

In people, high-resolution manometry (HRM) is considered the gold standard for the diagnosis of functional LES disorders such as LES-achalasia. Understanding the limitations of HRM in dogs, which include cost, availability, and patient compliance, our goal was to use a free-feeding VFSS protocol(125) to characterize VFSS features of functional LES obstruction. We defined a functional LES obstruction as a failure of the LES to relax in response to a pharyngeal swallow. Though a direct comparison between dogs and people cannot be made without manometry, we elected to refer to functional LES obstruction in our canine patients as LES achalasia-like syndrome (LES-AS) after the key pathophysiologic feature of achalasia in humans. $(178,186)$ The objective of this study was to identify VFSS parameters which could be used to identify LES-AS in dogs. We hypothesized that LES-AS could be distinguished from normal dogs using standardized VFSS criteria.

\section{Materials and Methods}

\section{$\underline{\text { Case Selection and Criteria }}$}

Medical records for dogs presenting to the University of Missouri (MU-VHC) between April 2015 and December 2017 for VFSS were retrospectively reviewed. Dogs were included if they had a standing, free-feeding VFSS available for review, a complete medical record, and evidence of a failure of the LES to relax in response to a pharyngeal swallow (LES-AS). Dogs determined to have LES-AS, were further evaluated for discriminating criteria between LES-AS, dogs with non-LES-AS ME, and healthy dogs. Common features among dogs with LES-AS were assessed to evaluate for discrete clinical syndromes.(178, 187) Standardized assessment and diagnostic criteria were 
developed and validated by calculating agreement between a panel of investigators at MU College of Veterinary Medicine and a blinded expert reviewer with expertise in the area of swallowing disorders (CPG) based on diagnostic criteria and characteristic features of disease identified in people. These criteria were inclusive of failure of the LES to relax in response to pharyngeal swallow, esophageal motility, retained ingesta/oral secretions within the esophagus, "bird-beak", reflux, and the absence of mechanical obstruction $(178,180,188,189)$ As objective metrics for determining ME involve comparisons to the cervical vertebrae or thoracic inlet, which may be out of view during parts of the VFSS, subjective assessment of dogs with all dogs with ME on VFSS was objectively evaluated by comparing the ratios of distal esophageal diameter (DeD) to the height of the $12^{\text {th }}$ thoracic vertebral body compared to healthy dogs. For those with focal ME the distal most portion of the esophagus orad to the obstruction was used to calculate the DeD.

Dogs receiving prokinetic medications or opioids and those with focal ME or VFSS evidence of mechanical obstruction (including hiatal hernia and esophageal strictures) were excluded. Additional testing for hypothyroidism, MG, and hypoadrenocortisolism were performed in most dogs at the discretion of the attending clinician based on supporting clinical evidence. Dogs with a positive diagnosis of hypothyroidism, MG, and/or hypoadrenocortisolism were not excluded from further evaluation. Swallow studies were compared against archived normal data from a previous publication ( $\mathrm{n}=20) .(125)$ Control dogs were considered healthy based on physical examination and the absence of either respiratory or gastrointestinal signs, including oral, pharyngeal, and esophageal dysphagia, for the 6 months prior to the VFSS.

Demographic Data 
Demographic data, clinical features, duration of clinical signs, incidences of aspiration pneumonia, and body condition scores were acquired from the medical record.

\section{Videofluoroscopic Swallow Study Protocol}

VFSS was performed in accordance with a previously validated free-feeding VFSS protocol.(125) Briefly, after a 12 hour fast, dogs were placed in 1 of 4 polycarbonate kennels designed to accommodate small/toy ( $\leq 16 \mathrm{~kg}$ ), medium ( $>16 \mathrm{~kg}-\leq 30 \mathrm{~kg})$, large ( $>30 \mathrm{~kg}-\leq 39 \mathrm{~kg}$ ), and giant breed ( $\geq 39 \mathrm{~kg}$ ) dogs. These kennels were designed to permit upright free feeding behavior, direct patient visualization, and contrast videofluoroscopy. The dogs were fed 3 standardized food consistencies containing a contrast agent; puree (25\% iohexol (350 mg/mL), liquid (25\% iohexol (350 mg/mL), kibble (barium $40 \%$ w/v)). Studies were performed at 30 frames/sec using a GE Advantx or GE OEC 9900 Elite Mobile C-Arm system at the MU-VHC. Studies were considered complete if they included VFSS views as described in Table 8. VFSS for functional LES-AS was considered diagnostic only if the LES could be evaluated in response to pharyngeal swallowing using multiple food/liquid consistencies containing oral contrast. The LES was actively challenged (contrast abutting the LES) during active swallowing with the dog in a sitting and/or standing position to mitigate the effect of esophageal weakness on the passage of contrast through the LES. Evaluation was also performed when the dog was not actively swallowing to assess for bolus passage secondary to hydrostatic pressure. Some dogs were asked to sit during active swallows to add extra challenge to the LES.

\section{$\underline{\text { Statistics }}$}

Statistical analysis was performed using MedCalc data analysis software (version 
18.5). Descriptive statistics were performed where appropriate. Non-parametric analysis was performed on objective swallow metrics due to the small sample size. Data are presented as median and interquartile range (IQR). A 95\% CI was calculated for common VFSS features of LES-AS. A ROC curve analysis was performed to determine the sensitivity and specificity for detection of ME using a ratio between the maximal distal esophageal diameter and the height of the T12 vertebral body. Kappa/weighted kappa coefficients were calculated to assess for agreement between the MU panel and the independent reviewer (CPG) for criteria discriminating between LES-AS and normal dogs and the assigned LES-AS subtype for each dog. Weighted kappa coefficients were weighted linearly. A P value of $\leq 0.05$ was considered significant.

\section{Results}

\section{$\underline{\text { Animals }}$}

One hundred thirty dogs underwent VFSS at the MU-VHC between April 2015 and December 2017. Twenty-nine patients were found to have ME based on VFSS. Megaesophagus was described as ether generalized $(n=23)$ or focal $(n=6)$. Those with generalized ME without LES-AS ( $\mathrm{n}=9$ ) were characterized by diffuse esophageal body hypomotility without LES obstruction. In these cases, a food bolus passed unimpeded into the stomach once presented to the LES. Timing of LES relaxation was coordinated with a pharyngeal swallow. Videofluoroscopic swallow study diagnosis for the 130 cases reviewed are available in Figure 4.

Out of 130 VFSS, 19 patients including $61 \%$ of those with generalized ME, met entry criteria for further evaluation by having a failure of the LES to relax in response to pharyngeal swallow. For those with LES-AS, ages ranged from 5 weeks to 12 years, with 
a median (IQR) age of 2.5 years (0.9-6.7 years). Seven dogs were spayed females, 2 were intact females, 8 were castrated males, and 2 were intact males. Breeds represented included Mixed breeds ( $\mathrm{n}=4$ ), Australian Shepherd ( $\mathrm{n}=3$ ), Chihuahua ( $\mathrm{n}=2)$, Golden Retriever ( $\mathrm{n}=2$ ), Miniature Schnauzer ( $\mathrm{n}=1)$, Miniature Dachshund $(\mathrm{n}=1)$, Doberman Pinscher ( $\mathrm{n}=1)$, German Shepherd Dog ( $\mathrm{n}=1)$, Irish Wolfhound $(\mathrm{n}=1)$, Boston Terrier $(\mathrm{n}=1)$, German Shorthair Pointer $(\mathrm{n}=1)$, and English Cocker Spaniel $(\mathrm{n}=1)$. The median (IQR) body condition score on a 9-point scale was 3 (2-5) with 4-5/9 being considered ideal.

Out of 19 dogs with LES-AS, presenting complaints included regurgitation $(n=14)$, regurgitation and cough $(n=4)$, and cough alone $(n=1)$. The duration of clinical signs prior to presentation ranged from 5 weeks to 4 years, with a median (IQR) of 7 months (4-16 months). Hypothyroidism was ruled out in 17/19 dogs by total T4/TSH. Hypoadrenocortisolism was ruled out in 17/19 dogs by either baseline cortisol ( $>2 \mathrm{ug} / \mathrm{dL}$, $>55 \mathrm{nmol} / \mathrm{L}$ ) or ACTH stimulation test. Acetylcholine receptor antibody testing was performed in 14/19 dogs. Testing was confirmatory for MG in $1 \mathrm{dog}$. In the remaining dogs, clinicians elected to forgo testing based on lack of supporting clinical signs. A previous history of aspiration pneumonia was reported in 5/19 dogs. No dog underwent anesthesia within 30 days of presentation to the VHC.

Archived VFSS from twenty research and companion dogs were included in our study as healthy controls ( $\mathrm{n}=20)$. Dogs were determined to be healthy based on history (no evidence of dysphagia, gastrointestinal or respiratory signs within 6 months of the VFSS) and physical examination. Ages ranged from 4 weeks to 14 years, with a median (IQR) age of 4.9 years (2.0-9.0 years). Eleven dogs were spayed females and 9 dogs 
were castrated males. Breeds represented included Pembroke Welsh Corgis $(\mathrm{n}=6)$, long haired Dachshunds ( $\mathrm{n}=5)$, Chinese Crested and Beagle mix ( $\mathrm{n}=5)$, large mixed breeds $(\mathrm{n}=2)$, Jack Russell Terrier $(\mathrm{n}=1)$, and German Shepherd Dog $(\mathrm{n}=1)$.

\section{$\underline{\text { Videofluoroscopic Swallow Studies }}$}

\section{$\underline{\text { MU Results }}$}

The VFSS metrics for evaluation are described in Table 9. Functional obstruction of the LES was diagnosed if there was failure of the LES to relax in response to a pharyngeal swallow during active challenge of the LES. VFSS videos were initially evaluated by a panel of trained reviewers including 2 board certified internal medicine specialists (MG, CR), a PhD and board-certified speech-language pathologist specializing in translational deglutology (TL), and a senior radiology resident (JS). Panel results were achieved by consensus. An MD gastroenterologist considered an expert in esophageal motility disorders (CPG) independently reviewed each study using the standardized criteria in Table 9. The independent reviewer (CPG) was blinded to the findings of the MU panel. The results of the MU panel and the independent reviewer were evaluated for agreement in order to validate our VFSS criteria for diagnosis and classification.

Multiple criteria for evaluation were used based on esophageal motility studies performed in people based on standardized criteria (bellow):(188, 190, 191)

1) Megaesophagus: Dogs were assessed for the presence/absence of generalized esophageal dilation. Subjective evaluation was subsequently compared to objective parameters (esophageal diameter at its widest point comparted to the height of the T12 vertebral body). T12 was selected for ease of visualization relative to the LES. (Figure 5) 
2) Timing of ingesta entry into the stomach: Movement of the contrast bolus from the distal esophagus into the stomach was assessed to determine if bolus passage was in response to active pharyngeal swallowing, secondary to hydrostatic pressure (i.e., gravity-dependent and not during swallowing), or due to a secondary peristaltic wave.

3) Baseline fluid line: Prior to administering contrast-laden food or liquid, the esophagus was assessed for the presence/absence of fluid retention within the esophagus after a prolonged fast ( $\geq 12$ hours). This is distinguished from reflux by evaluating for persistence/lack of clearance. Ventrally dependent fluid within a diverticulum was not considered positive for a fluid line. (Figure 6)

4) LES "bird-beak": The distal esophagus and LES were assessed for the presence/absence of a dilated distal esophagus terminating in an elongated taper ("bird-beak") though the LES during active pharyngeal swallowing. (Figure 7)

5) Esophageal peristalsis (contraction and propulsion): The esophagus was assessed for presence/absence of the following clinical features:

a. Primary peristalsis was defined as a wave of bolus movement beginning in the proximal esophagus, initiated by a pharyngeal swallow.

b. Secondary peristalsis was defined as a wave initiated by esophageal distention, evaluated while the dog was not actively eating/drinking to avoid confounding by concurrent primary peristalsis and clearance initiated by a subsequent food bolus.

c. Esophageal contraction referred to the inward movement of the dorsal and ventral esophageal walls. 
d. Propulsion refers to the ability of either primary or secondary peristaltic activity to conduct a food bolus aborally towards the LES.

6) Narrowed (spastic) distal esophagus: The distal esophagus was assessed for the presence/absence of a transient segmental decrease in diameter of the distal esophagus, proximal to the LES, resulting in a narrowed contrast column. A lack of robust contractions against a closed LES distinguishes this finding from a hypermotile esophagus. (Figure 8a, b)

7) Hypermotile distal esophagus: The distal esophagus was assessed for the presence/absence of robust contraction against a closed LES, during or between pharyngeal swallows.

8) Reflux: Presence/absence of orad movement of contrast from the stomach into the esophagus was evaluated passively and during forced abdominal compression. During abdominal compression, a licensed veterinarian wearing appropriate personal protective equipment applied abdominal pressure to a standing dog in order to induce reflux and/or sliding hiatal hernia.

9) Hiatal hernia: Presence/absence of herniation of the stomach into the thoracic cavity (through the esophageal hiatus of the diaphragm) was assessed either passively or in response to abdominal pressure by a licensed veterinarian. ME was present in 14/19 dogs with LES-AS. The ratio of the maximum distal esophageal diameter (DeD):height of T12 vertebral body being significantly greater in all dogs with subjective ME (n=29) (median:6.4 IQR:6.0-7.3) compared to healthy dogs (median 3.8 IQR: $3.3-4.0 ; \mathrm{p}<0.001$ ). No statistically significant differences were identified between LES-AS and non-LES-AS dogs with ME for the DeD: height of T12. 
A DeD:T12 ratio greater than 4.8 was $94 \%$ sensitive and $100 \%$ specific for ME compared to normal controls. In dogs with LES-AS, a baseline esophageal fluid line was present in 68.4\% (95\% CI, 47.5-89.3\%). An LES “bird-beak" was present in 63.2\% (95\% CI, 41.5 84.8\%) of dogs with LES-AS. These features were not present in any dog with ME that did not also have LES-AS. Additional discriminatory VFSS criteria for LES-AS compared to controls available in Table 10.

For primary peristalsis, the esophagus was subjectively graded by the MU panel as acontractile $(\mathrm{n}=8)$, hypomotile $(\mathrm{n}=8)$, or hypermotile $(\mathrm{n}=3)$ compared to normal controls. Normal primary peristaltic propulsion was not identified in any LES-AS dog. Apart from 1 dog, in dogs with acontractile or hypomotile primary peristalsis, decreased secondary peristalsis was also observed. Normal secondary contractions $(n=4)$ diffuse throughout the length of the esophagus were observed in dogs with hypermotile primary peristalsis and in the distal esophagus of 1 dog with hypomotile primary peristalsis. Reflux was identified in 1/19 dogs with achalasia-AS compared to $8 / 20$ of healthy controls. In healthy dogs, physiologic reflux occurred commonly during feeding but tended to be restricted to the distal esophagus and was rapidly cleared by a subsequent food bolus. In our clinical experience, clinical patients where reflux is thought to be pathologic, reflux tends to be spontaneous (not during feeding), may span the length of the esophagus, or is retained in the esophagus for a longer period. The one dog with LESAS with reflux appeared to have physiologic reflux, excepting that reflux events occurred spontaneously (i.e., not during feeding). This dog was later diagnosed with a distal mechanical obstruction of the esophagus (pseudoachalasia). Post-procedural aspiration pneumonia was not reported for any dog undergoing VFSS. 
In evaluating VFSS in dogs with LES-AS, classification criteria for three discrete syndromes was developed, and a case of pseudoachalasia was identified. While each syndrome was characterized by failure of the LES to relax in response to pharyngeal swallowing, there were differences in the degree and type of peristaltic dysfunction, as well as the degree of gastric filling and esophageal dilation.

Type 1 LES-AS ( $\mathrm{n}=8$ dogs) showed ME with acontractile primary peristalsis and absent secondary contraction. Abnormal LES relaxation (failure to relax in response to pharyngeal swallowing) was observed in response to all food/liquid types, resulting in minimal gastric filling.

Type 2 LES-AS ( $\mathrm{n}=7$ dogs) was characterized by hypomotile primary peristalsis with or without ME. Of note, increased hydrostatic pressure (achieved by sitting, upright feeding, or significant accumulation of food within the esophagus) facilitated gastric filling.

Type 3 LES-AS ( $n=3$ dogs) demonstrated a spastic distal third of the esophagus or a hypermotile distal esophagus against a closed LES, with or without ME.

a. Spasticity was defined as a transient, segmental decrease in diameter of the distal esophagus, proximal to the LES, resulting in narrowed contrast column.

b. Hypermotility was defined as robust contractions against a closed LES, during or between pharyngeal swallows.

$\underline{\text { Pseudoachalasia }(n=1)}$ was defined as mechanical esophageal-gastric junction outflow obstruction. In the dog in this report it was due to a circumferential adenocarcinoma.

Objective (DeD:T12) and subjective interpretations of ME were found to have 
perfect agreement. After agreement was achieved among the $\mathrm{X}$ panel, discriminatory and sub-classification criteria were compared to the review performed by the MD gastroenterologist to calculate agreement. Specific kappa values, standard error, and 95\% confidence intervals are presented in Table 11.

\section{Discussion}

This study has demonstrated that a subpopulation of dogs with ME have a functional obstruction of the LES (LES-AS), which may open the door to additional therapeutic opportunities. A lack of understanding of disease pathogenesis and limitations in available diagnostics play a role in the poor prognosis associated with ME. This manuscript details VFSS characterization of functional LES obstruction, termed LES-AS in dogs. The identification of a previously overlooked subpopulation of dogs with esophageal dysphagia provides hope to these patients, as humans with analogous diseases may respond to interventions targeting functional obstruction of the LES including injection of the LES with botulinum toxin A, pneumatic LES dilation, or surgical LES myotomy. Though further research is needed, the identification of similar clinical syndromes between dogs and people may suggest utility for dogs with LES-AS as a translational model for humans with LES achalasia. As knowledge is bi-directional, successful treatment protocols for humans may also benefit our canine species and merit evaluation in clinical trials.

The gold standard for evaluation of LES achalasia in people is HRM, which evaluates esophageal motor function by detecting esophageal and LES pressure profiles.(178-180,192) In humans, this technique allows the detection and subcategorization of obstructive LES disorders based on their pathophysiologic profiles. 
(179) This technique is rarely utilized in companion animals due to limited availability, high cost, and variable patient tolerance. $(18,193,194)$ Conventionally, evaluation of dysphagia in dogs has relied upon historical information, physical examination findings and radiography including VFSS, all of which are considered the gold standard in veterinary medicine.(125) In people, swallow studies were used prior to the development of HRM to detect LES achalasia and have more recently been proposed as the method of choice for detecting recurrence of functional LES obstruction after therapy.(180, 188, 191) The barium esophagram is an established protocol for detecting LES achalasia and is considered an alternative initial approach in humans where LES achalasia is suspected.(178, 180, 191) Standardized dynamic contrast studies, such as the "timed barium swallow," allows clinicians to detect the degree of esophageal bolus retention.(188) Despite only moderate sensitivity, these tests are considered specific for a diagnosis of LES achalasia.(191) Prior to our VFSS-based study, there had been no such standardized protocols developed for evaluating functional LES obstruction in dogs. In part, this may be due to the paucity of VFSS in dogs with ME and other forms of dysphagia, for which the historical protocol of restrained recumbency and force feeding of contrast has unacceptable risks of aspiration.(195, 196) However, allowing upright free-feeding in unrestrained dogs dramatically diminishes the risk of aspiration to no more than what would be encountered during feeding at home and allows the study of any type of dysphagia (i.e., oral, pharyngeal, and/or esophageal).(125) This technique also allows the investigator to assess the timing of LES relaxation in response to pharyngeal swallow allowing investigators to distinguish between LES-AS and other forms of ME. These are broadly characterized by esophageal hypomotility/weakness 
without functional obstruction of the LES. This distinction is critically important as the functional obstruction at the level of the LES is what determines candidacy for targeted therapeutic intervention. In our clinic, no dog having undergone a free feeding VFSS developed post-procedural aspiration pneumonia. Our VFSS findings represent a substantial departure from a prior study where VFSS performed in lateral recumbency failed to identify any dog with functional LES obstruction compared to $61 \%$ of our patients with ME (Figure 4).(196) Our findings suggest that this procedure can be performed safely in dogs with ME and esophageal dysphagia to identify dogs with functional LES disorders.

Abnormal relaxation of the LES in response to pharyngeal swallowing is recognized as the key pathophysiologic feature in LES achalasia.(178-180,192) This highlights the need for evaluation of the LES during active swallowing in order to assess for inappropriate failure of the LES to relax. Several swallows should be evaluated for each food/liquid consistency, as LES achalasia may not occur with every swallow. Common features of LES achalasia in people undergoing dynamic imaging include ME, the distal esophagus/LES "bird-beak", a lack of primary peristalsis, and a persistent contrast column above the LES.(180) As a result of this functional obstruction, the esophageal body can lose tone and dilate, retaining both ingesta and oral/respiratory secretions. In our study, these clinical features were also identified in dogs with LES-AS. Interestingly, though ME was commonly identified, 5/19 dogs with LES-AS had objectively and subjectively normal esophageal diameter compared to controls. As such, the absence of ME does not rule out LES-AS in dogs and should be considered as a differential in dogs with signs of esophageal dysphagia despite the absence of ME. Serial 
evaluation of these dogs would be warranted to determine if they progress to development of ME. Based on this study subjective and objective assessment of ME in dogs compared to healthy dogs had perfect agreement suggesting that objective calculations are not always necessary to confirm diagnosis of $\mathrm{ME}$ and objective measurement may be reserved for cases where ME may be considered borderline. However, the lack of a gold standard measurement for ME is a limitation in calculating specificity for dogs in this study. A baseline fluid line and bird-beak were frequently identified in patients with LES-AS and was absent in normal dogs and in our population of dogs with other forms of ME. This may suggest that fluid line and bird-beak could discriminate LES-AS from forms ME, though further study is needed. Two cases of seropositive MG were identified in our population. One with LES-AS and the other with diffuse esophageal hypomotility without LES obstruction. A previously published case report used manometry to confirm a functional LES obstruction in a pug with seronegative MG.(194) This suggests that functional obstruction of the LES may be a feature of ME in some, but not all patients with MG. However, too little data exists to speculate on the pathophysiology of ME in these patients.

In humans, LES achalasia represents a family of syndromes grouped by variations in esophageal contractility rather than a single disorder characterized by a sole discrete phenotype.(178-180) The subtype of LES-AS in people does not determine candidacy for targeted intervention. It may however be used to help determine which means of LES disruption is selected (e.g. pneumatic dilation vs. BTA injections, or surgery) as different subtypes may have different response rates. $(179,186,187,192)$ Three phenotypes were characterized in our canine patient population which share 
similarities to the syndromes described in people. (178-180) As in people, Type 1 was considered end stage with absence of esophageal motility, and presence of esophageal dilation and minimal gastric filling. Type 2 was characterized by esophageal hypomotility. Retention of some esophageal tone and biophysical processes which allowed for improved esophageal emptying and gastric filling compared to Type 1, especially gravity-dependent increases in hydrostatic pressure. Type 3 LES-AS demonstrated impaired distal esophageal and LES relaxation in response to a food bolus resulting in esophageal bolus retention despite vigorous esophageal contraction upstream to the LES.(179) Like HRM, VFSS may result in a false positive diagnosis for patients with a distal mechanical LES obstruction (i.e. pseudoachalasia). In this study, one dog who was initially classified as having Type 3 achalasia was later identified to have a circumferential LES adenocarcinoma, resulting in a mechanical LES obstruction. Interestingly, this was also the only clinical dog with detectable reflux compared to $8 / 20$ asymptomatic healthy controls. Though speculative, pseudoachalasia may have resulted in an LES with a fixed diameter that simultaneously impeded gastric filling and permitted small volumes of spontaneous reflux. This form of pseudoachalasia has been previously identified in humans(197) and underscores the need for adjunctive diagnostics such as esophagoscopy to evaluate for occult mechanical obstructions which may mimic LES-AS prior to targeted intervention.(192)

Given the clinical importance of ME and esophageal dysphagia in dogs, it is critical that patients who may be receptive to targeted therapy be accurately identified. For this reason, VFSS images were interpreted by a blinded, independent MD reviewer who participated in the international working group for disorders of gastrointestinal 
motility and function to develop the consensus statement on achalasia syndromes in humans. Our goal was to adapt this diagnostic criteria and gauge the ease by which it could be applied to clinically affected dogs.(186) Agreement between the MU panel and the MD reviewer was perfect for 6/17 VFSS parameters, near perfect agreement for 5/17 parameters, substantial for $4 / 17$ parameters, moderate for $1 / 17$ parameters, and fair for 1/17 parameters.(198) (Table 11) This suggests that with training, these criteria could be applied by others to detect the subpopulation of dogs affected by LES-AS, thus broadening the ability of veterinarians to detect patients that may respond to targeted intervention. It should be noted that only fair agreement was reached for the frequency of abnormal swallow events (failure of the LES to relax in response to pharyngeal swallow). This finding supports our earlier recommendation that multiple swallow events should be evaluated because, depending on the LES-AS subtype and observer, aberrant swallowing events may appear variably frequent. However, this did not impact the agreement between the MU panel and the independent reviewer (CPG) for the final diagnosis of LES-AS for any dog.

\section{Conclusions}

Due to its inherent limitations, HRM for diagnosis of LES-AS does not currently have significant clinical utility outside of a few veterinary research centers or tertiary care facilities. The morbidity and mortality of ME in dogs necessitated developing a more accessible diagnostic test for identifying dogs that may respond to targeted intervention. Compared to HRM, VFSS is relatively accessible, inexpensive, and with training, easy to perform. In keeping with our stated objective, this study demonstrates that functional LES obstructions (LES-AS) can be identified by VFSS. We have also demonstrated that 
VFSS could identify discrete achalasia syndromes as are appreciated in humans.

Interpretation will require high quality diagnostic studies and practice, with the guidelines developed herein highlighting important features of LES-AS. Identifying this previously unrecognized patient population allows future exploration of treatments focusing on relieving functional obstruction of the LES to reduce morbidity and mortality associated with canine ME and esophageal dysphagia. 


\section{CHAPTER 4}

\section{MECHANICAL DILATION AND BOTULINUNM TOXIN A (BTA) INJECTIONS FOR TREATMENT OF LOWER ESOPHAGEAL SPHINCTER ACHALASIA-LIKE SYNDROME IN DOGS}

\section{Introduction}

Megaesophagus (ME) in dogs is a motility disorder of the esophagus that carries a poor long-term prognosis with death frequently reported secondary to respiratory complications, malnutrition or both, or euthanasia because of poor quality of life.(172174) For dogs with idiopathic ME, interventions are limited in part because of a lack of understanding of the underlying disease mechanisms. Recently, using a free-feeding videofluoroscopic swallow study (VFSS) protocol(125), we identified a subpopulation of dogs with functional obstruction of the lower esophageal sphincter (LES) analogous to LES achalasia in people.(148) In people, LES achalasia is diagnosed by high resolution manometry (HRM) and is characterized by a failure of the LES to relax in response to pharyngeal swallowing.(178) This functional obstruction results in esophageal dilation, retention of ingesta and oral secretions in the esophagus, loss of esophageal motility, and associated clinical signs of esophageal dysphagia.(178, 179, 199) Like achalasia in people, dogs with LES-AS lacked LES relaxation in response to pharyngeal swallow on VFSS.(148) Identifying LES achalasia in dogs is critically important because, in people, it may respond to treatment targeting the LES and esophageal outflow obstruction.(178, 180, 187, 200-202) The analogous functional LES obstruction in dogs, LES achalasialike syndrome (LES-AS), likewise may represent a condition responsive to targeted intervention, though therapeutic studies in this population are critically lacking. 
In people, achalasia is subcategorized into a spectrum of different disease phenotypes by HRM. Unfortunately, routine use of HRM in veterinary patients is impractical because of high cost, limited availability, poor patient compliance, and the need for substantial operator expertise. $(18,193)$ These limitations led to VFSS being successfully evaluated as a surrogate for the diagnosis of functional LES obstruction in dogs.(148) In people, regardless of clinical phenotype or method of diagnosis, the core treatment objective is to relieve the esophageal obstruction by mechanical disruption of the LES or lowering LES tone. In people, positive clinical responses are seen after mechanical dilation (pneumatic dilation or bougienage), botulinum toxin A(BTA) injections, LES myotomy with fundoplication or some combination of these.(178, 180, 187, 200-202) Given the positive response to targeted therapy in people with LES achalasia, our objective was to evaluate the response of dogs with LES-AS to targeted intervention with mechanical dilation and LES botulinum toxin A (BTA) injections, with or without surgical myotomy with fundoplication. We hypothesized that clinical and VFSS features of LES-AS would improve after treatment targeting the LES and esophageal outflow obstruction.

\section{Materials and Methods}

\section{$\underline{\text { Case Selection and Criteria }}$}

Dogs presented to the University of Missouri Veterinary Health Center (MUVHC) between April 2015 and December 2017 had medical records retrospectively reviewed. Dogs were included if they had complete medical records, were diagnosed with LES-AS by free-feeding VFSS and underwent targeted treatment by mechanical LES dilation (pneumatic dilation or bougienage), LES BTA injections, with or without LES 
myotomy with fundoplication. Dogs receiving prokinetics or opioids, those with a secondary form of ME, and those with focal ME or evidence of mechanical obstruction (e.g. pseudoachalasia, stricture, hiatal hernia) at the time of diagnosis were excluded. For most dogs, additional testing for relevant endocrinopathies (hypothyroidism, hypoadrenocortisolism) and myasthenia gravis was performed at the discretion of the attending clinician, based on supporting clinical evidence. Dogs meeting inclusion criteria were evaluated for a number of clinical and VFSS outcome variables to determine response to treatment (Table 1). The VFSS features at the time of diagnosis were used to compare post-treatment VFSS outcomes for each dog. Dogs with positive response to LES mechanical dilation with BTA injections were considered candidates for and offered the option of surgical intervention (LES myotomy with fundoplication) as a longer-term treatment.

\section{Data Extracted from the Medical Record}

Demographic data, clinical features, prior medical management for ME, duration of clinical signs, clinical perception of post-treatment disease control (positive or negative treatment response), body weight (BW), body condition score (BCS), frequency of regurgitation and complications after treatment were retrieved from the medical record or from follow-up calls to clients. Body condition score was evaluated according to the American Animal Hospital Association guidelines. Clients were requested to quantify frequency of regurgitation (episodes per day) before treatment as part of pre-treatment evaluation and after treatment as part of follow-up evaluation. No changes were made to the patient's pre- treatment regimen after targeted intervention to avoid confounding treatment effects. 


\section{Videofluoroscopic Swallow Studies}

Videofluoroscopic swallow studies were performed both to confirm diagnosis of LES-AS and 2-3 weeks post-mechanical dilation and BTA injection as previously described.(125) Briefly, after being fasted for 12 hours, dogs were placed in 1 of 4 kennels selected according to patient body size. The polycarbonate kennels are designed to accommodate small or toy ( $\leq 35 \mathrm{lbs}$ ), medium ( $>35 \mathrm{lbs}$ to $\leq 65 \mathrm{lbs})$, large ( $>65 \mathrm{lbs}$ to $\leq 85 \mathrm{lbs}$ ), and giant breed ( $\geq 85 \mathrm{lbs}$ ) dogs. These kennels were designed to permit unrestrained free-feeding behavior, maximize ease of patient visualization, and perform contrast videofluoroscopy. Dogs were fed 3 standardized food consistencies containing a contrast agent: puree (25\% iohexol [350 mg/mL]), liquid $(25 \%$ iohexol [350 mg/mL]), and kibble (barium $40 \% \mathrm{w} / \mathrm{v}$ ). Studies were performed at 30 frames/sec using a GE Advantx or GE OEC 9900 Elite Mobile C-Arm system (GE Heathcare, Chicago, IL) at the MU-VHC. VFSS videos were evaluated by a panel of trained reviewers including 2 board certified internal medicine specialists (Megan Grobman, Carol Reinero), a PhD and board-certified speech-language pathologist specializing in translational deglutology (Teresa Lever), and a senior radiology resident (James Schachtel). The VFSS was considered diagnostic for LES-AS if a lack of LES relaxation was observed in response to pharyngeal swallowing. The LES was actively challenged (contrast abutting the LES) during active swallowing with the dog in a sitting or standing position or both to mitigate the effect of esophageal weakness on the passage of contrast through the LES. Evaluation also was performed when the dog was not actively swallowing to assess bolus passage secondary to hydrostatic pressure. Some dogs sat during active swallows to add additional challenge to the LES. The VFSS parameters used for pre- and post-treatment 
comparisons were selected based on studies in humans showing improved gastric filling, improved ME, and improved esophageal motility after treatment for achalasia.(180, 188, 203) The VFSS outcome parameters are provided in Table 12.

1) ME: Dogs were assessed pre- and post- treatment for subjective changes in esophageal diameter.

2) Esophageal motility and peristalsis (contraction and propulsion): The esophagus was assessed for presence or absence of the following clinical features:

a. Primary peristalsis, defined as a wave of bolus movement beginning in the proximal esophagus, initiated by a pharyngeal swallow.

b. Secondary peristalsis, defined as a wave initiated by esophageal distention, evaluated while the dog was not actively eating ordrinking to avoid confounding by concurrent primary peristalsis and clearance initiated by a subsequent food bolus.

c. Esophageal contraction referred to as the inward movement of the dorsal and ventral esophageal walls. Dogs without VFSS evidence of contraction were referred to as "acontractile."

d. Propulsion referred to the ability of either primary or secondary peristaltic activity to conduct a food bolus aborally towards the LES.

e. Amotile: referred to dogs without evidence of primary or secondary peristalsis

f. Hypomotile: referred to dogs with evidence of primary and/or secondary peristaltic waves that are unable to conduct a food bolus aborally toward the LES. 
g. Hypermotile: referred to spastic or excessive esophageal motility

i. Spasticity: transient segmental decrease in the esophageal diameter, proximal to the LES and resulting in a narrowed contrast column.

ii. Excessive motility: robust contraction against a closed LES, during or between pharyngeal swallows.

h. Normal motility: referred to normal primary and secondary peristalsis that transferred swallowed boluses unimpeded to the LES.

3) Gastric filling: Passage of ingesta into the stomach in response to pharyngeal swallowing or hydrostatic pressure (sitting or standing). If residual food or contrast remained in the esophagus, dogs were held upright for 5 minutes to increase hydrostatic pressure and facilitate emptying into the stomach.

a. The extent of gastric filling was evaluated pre- and post- treatment and graded as small $(<25 \%)$, medium $(25-75 \%)$, or large $(>75 \%)$.

\section{$\underline{\text { Targeted Intervention for LES-AS }}$}

All procedures requiring general anesthesia (endoscopy, mechanical disruption of the LES [pneumatic dilation and bougienage], BTA injections of the LES, and LES surgery) were performed a minimum of 12 hours after VFSS. Anesthetic protocols and monitoring were performed under the direction and supervision of a board-certified anesthesiologist.

\section{Endoscopy}

Esophagoscopy and abbreviated gastroscopy were performed using a Fujinon EG450HR, $10.7 \mathrm{~mm}$ gastroscope (Fujifilm, Wayne, New Jersey). Endoscopy was performed 
before to mechanical dilation and BTA injections to evaluate for evidence of an occult mechanical obstruction of the LES. Esophagoscopy included evaluation of the esophageal body for wall defects, mucosal changes, and residual food or fluid. The LES and cardia were assessed for a distal esophageal stricture and to determine the ease of passage of the endoscope through the LES. Strong resistance to passage of the endoscope was considered suspicious for mechanical obstruction of the LES (pseudoachalasia).(204) A "J maneuver" was performed to evaluate for pseudoachalasia capable of causing esophageal outflow obstruction. Because a diagnosis of LES-AS was made based on a failure of LES relaxation in response to pharyngeal swallowing, an open LES observed under anesthesia was not considered to contradict a VFSS diagnosis of LES-AS nor was it a contraindication to targeted therapy. Fluid and food were suctioned from the esophagus to permit visualization before mechanical LES dilation and BTA injections.

\section{$\underline{\text { Mechanical Dilation }}$}

Mechanical dilation was performed either by pneumatic dilation (CRE ${ }^{\mathrm{TM}}$ Pro Wireguarded Balloon Dilation Catheter, Boston Scientific, Marlborough, MA) or bougienage. Balloon diameter, ranging from 1-3 cm, was subjectively adjusted according to patient size to prevent overdistension and perforation. Under endoscopic guidance, the balloon was passed through the LES (205) making sure to span the entire length, inflated, and then held in place for 90 seconds. This process was repeated 2-3 times. Blanching of the mucosa at the LES was observed through the transparent balloon (Figure 9). The endoscope did not simultaneously span the LES during deployment of the balloon to allow uniform radial force to be applied to the LES. In $1 \mathrm{dog}$, after the only appropriately sized available balloon was determined to be damaged, rubber bougies (40-50 French) 
were inserted sequentially through the LES and each held in place for 90 seconds. This process was repeated twice. Confirmation of bougie placement and mucosal blanching were performed as for pneumatic dilation.

\section{$\underline{\text { BTA Injection }}$}

After mechanical dilation, BTA (Botox [onabotulinumtoxinA] ${ }^{\circledR}$, Allergan, Madison, NJ) diluted to $40 \mathrm{U} / \mathrm{mL}$ in $0.9 \%$ sterile saline was administered using an endoscopic injection needle (Interject ${ }^{\mathrm{TM}}$ Sclerotherapy Needle, Boston Scientific, Marlborough, MA) at 8 sites around the LES (4 U/site) (Figure 10a, b). The first 4 injections (Set 1 ) were made circumferentially at $90^{\circ}$ intervals at the esophagogastric junction. The second 4 injections (Set 2) were made $1 \mathrm{~cm}$ distal to Set 1, also circumferentially at $90^{\circ}$ intervals. Set 2 was rotated $45^{\circ}$ from Set 1 , as shown in Figure 10b. A small bleb was visible after each injection with no visually detectable losses.

\section{$\underline{\text { Surgical LES Myotomy with Fundoplication }}$}

Surgical myotomy of the LES (Heller procedure) was performed followed by Dor fundoplication as previously reported in the human surgical literature.(206) A standard ventral midline celiotomy was performed and the LES was isolated from its attachments within the crus of the diaphragm. The definitive location of the LES was determined by intra-operative endoscopy $(5 \mathrm{~mm} \times 65 \mathrm{~mm}$ Storz Bidirectional Intubation Fiberscope, Tuttlingen, Germany) and marked with monopolar electrosurgery on the serosa of the stomach at the esophagogastric junction. A full thickness myotomy of the LES was performed extending $3 \mathrm{~cm}$ orad in the esophagus and $3 \mathrm{~cm}$ aborad in the stomach. Complete myotomy of the LES was confirmed by retromucosal illumination via intraoperative endoscopy to assess for residual muscle fibers overlying the submucosa. After 
completion of the myotomy, a Dor fundoplication was performed using polypropylene suture. The right and left crura of the diaphragm were apposed and residual air within the thoracic cavity was removed by suction. Finally, adequate patency of the LES was confirmed by endoscopic visualization before closure. A gastric tube was placed to facilitate feeding as needed during recovery. Post-operative analgesic protocols were carried out at the discretion of the attending clinician. All dogs were treated postoperatively with omeprazole ( $1 \mathrm{mg} / \mathrm{kg}$ PO q $12 \mathrm{~h}$ ) for 10-14 days.

\section{Statistics}

Statistical analysis was performed using SigmaPlot data analysis software (version 14.0). Descriptive statistics were performed where appropriate. Non-parametric analysis was performed because of the small sample size. Wilcoxon Signed Rank tests were performed on pre-and post-treatment variables of BW, BCS, and frequency of regurgitation. Pre-treatment data was collected from dogs with LES-AS at the time of diagnosis. Post-treatment data was collected at the time of the first evaluation after mechanical dilation and BTA injections. Data are presented as median and interquartile range (IQR). A $p$ value of $\leq 0.05$ was considered significant.

\section{Results}

\section{$\underline{\text { Animals }}$}

One-hundred and thirty VFSS were performed at the MU-VHC between April 2015 and December 2017 (Figure 4). Nineteen dogs were diagnosed with LES-AS based on VFSS criteria and 14/19 met inclusion criteria for the study. Ages ranged from 5 weeks to 12 years with a median (IQR) age of 2.5 years (0.9-5.8 years). Five dogs were spayed females, 3 were intact females, 2 were castrated males, and 4 were intact males. Breeds represented included mixed breeds $(\mathrm{n}=3)$, Australian Shepherd $(\mathrm{n}=2)$, Chihuahua 
$(\mathrm{n}=1)$, Golden Retriever $(\mathrm{n}=1)$, Miniature Schnauzer $(\mathrm{n}=1)$, Miniature Dachshund $(\mathrm{n}=1)$, Doberman Pinscher ( $\mathrm{n}=1)$, German Shepherd Dog $(\mathrm{n}=1)$, Irish Wolfhound $(\mathrm{n}=1)$, German Shorthair Pointer $(\mathrm{n}=1)$, and English Cocker Spaniel $(\mathrm{n}=1)$.

Presenting complaints included regurgitation $(\mathrm{n}=11)$, regurgitation and cough $(\mathrm{n}=2)$, and cough alone $(\mathrm{n}=1)$. The duration of clinical signs before presentation ranged from 5 weeks to 4 years with a median (IQR) of 8 months (4-18 months). Twelve of 14 dogs had $\geq 2$ weeks medical management for regurgitation before presentation including upright feeding and treatment with proton pump inhibitors with or without other gastroprotectants. Thirteen of the 14 dogs had ME at the time of diagnosis for LES-AS. Hypothyroidism was ruled out in 12/14 dogs by total thyroxine (T4) and thyroid stimulating hormone (TSH) concentrations. Hypoadrenocortisolism was ruled out in 12/14 dogs either by baseline serum cortisol concentrations ( $>2 \mu \mathrm{g} / \mathrm{dL},>55 \mathrm{nmol} / \mathrm{L})$ or ACTH stimulation test. Acetylcholine receptor antibody testing was performed and found to be negative in 12/14 dogs. In the remaining dogs, clinicians elected to forgo testing based on lack of supporting clinical signs. A previous history of aspiration pneumonia was reported in $5 / 14$ dogs.

\section{Endoscopy}

Esophagoscopy and abbreviated gastroscopy were performed uneventfully in all 14 dogs. Esophageal diverticula were identified at the thoracic inlet in 2/14 dogs corresponding to lesions observed on VFSS. Bone fragments and ingesta were present in the dependent region of the diverticulum in 1 dog. Despite being fasted $\geq 12$ hours, esophageal fluid was present in all 14 dogs. Roughened texture and esophageal hyperemia were observed in all 14 dogs. According to our inclusion criteria, no evidence 
of mechanical obstruction was identified in any dog.

\section{$\underline{\text { Post- Mechanical Dilation and BTA Injection (Clinical Variables) }}$}

Dogs were presented for follow-up a median (IQR) of 21 days (14-25 days) posttreatment. Total post-treatment follow-up was a median (IQR) of 3.5 months (2-4.8 months). At the time of the first post-treatment evaluation, $100 \%$ of owners described subjective clinical improvement. Body weight was significantly $(\mathrm{P}<0.001)$ increased post-treatment. Median (IQR) pre- and post- treatment body weights $(\mathrm{kg})$ were $7.3 \mathrm{~kg}$ (5$15.8 \mathrm{~kg})$ and $8.1 \mathrm{~kg}(4.7-23.25 \mathrm{~kg})$, respectively. Median (IQR) percent increase in body weight was $20.4 \%$ (12.7-25). No dog lost weight during the evaluation period. Median body condition score also was significantly $(\mathrm{P}<0.001)$ increased after treatment. Median (IQR) pre- and post-treatment BCS (9 point scale) were 3 (3-3.5) and 5 (4-5) respectively. Frequency of regurgitation was significantly $(\mathrm{P}<0.001)$ decreased post-treatment. The median (IQR) decrease in regurgitation as recorded by owners was $80 \%(50-85 \%)$. Median (IQR) duration of effect was 40 (17-53) days.

\section{$\underline{\text { Post- Mechanical Dilation and BTA Injection (VFSS Parameters) }}$}

Pre- and post- treatment VFSS findings are presented in Table 13. After LES mechanical dilation with BTA, all 14 dogs lacked detectable change to esophageal diameter or motility. Gastric filling was markedly improved in 12/14 dogs following treatment (Figure $11 \mathrm{a}, \mathrm{b})$.

\section{Complications Post-BTA Injection with Mechanical Dilation}

Complications following BTA and mechanical dilation were reported for 2/14 dogs. One dog developed post-treatment aspiration pneumonia. This dog responded well to medical management and recovered uneventfully. No long-term consequences related 
to aspiration pneumonia were identified. Improvement in clinical signs and VFSS were observed for this dog after recovery from aspiration pneumonia. In another dog, a gastroduodenal-esophageal intussusception with a Type IV hiatal hernia was identified 3 weeks after mechanical dilation with BTA injections (Figure $12 \mathrm{a}-\mathrm{d}$ ). The dog underwent surgery in which the stomach, spleen and a portion of the duodenum and pancreas were identified in the distal esophagus. The hernia was surgically corrected during exploratory celiotomy and left-sided gastropexy. Substantial improvement in clinical signs had been recorded for this dog before it developed complications.

\section{$\underline{\text { Heller Myotomy and Dor Fundoplication }}$}

Dogs with documented improvement after mechanical dilation with BTA injections were considered candidates for surgical intervention. Six of 14 dogs underwent surgery (Heller myotomy with Dor fundoplication). The median (IQR) follow-up for patients undergoing Heller myotomy with Dor fundoplication was 7 months (1-21 months). In all 6 dogs, post-surgical clinical signs and VFSS features were similar to those at the first evaluation after mechanical dilation and BTA injections (i.e. improved over baseline). In addition, 2 dogs had improvement in esophageal diameter and esophageal motility as measured by VFSS $>6$ months postoperatively, indicative of a delayed positive functional response to surgery. Of the dogs that showed improved motility, 1 dog was considered to have an amotile esophagus and the other a hypomotile esophagus. Evaluation of response with respect to patient age, duration of clinical signs, and LES substage could not be performed because of to our small sample size.

\section{Discussion}

Idiopathic ME in dogs is a disorder with high morbidity and mortality that historically has lacked effective targeted treatment. A subgroup of these dogs with LES- 
AS have excellent clinical responses to mechanical dilation and BTA injections, with surgery being a more definitive, long-term option. To identify which dogs with ME may benefit from these targeted treatments, use of a free-feeding VFSS protocol is crucial to identify functional LES obstruction.(148) In people with LES achalasia, relieving the esophageal-gastric outflow obstruction significantly improves clinical signs.(178-180, 201, 207) Comparably, significant clinical improvement, based on our previously described outcome variables, was observed in our population of dogs with LES-AS that underwent targeted intervention using mechanical dilation and BTA injections, although the response was temporary and shorter than is typical in people.(192, 205, 206) Surgery provided sustained clinical improvement compared to baseline, despite not resolving the ME and esophageal motility defects detected by VFSS. In all dogs, VFSS showed improvement in gastric filling after surgery. By promoting gastric filling, improvement in the aforementioned outcome variables (body weight and body condition score as well as decreased regurgitation frequency) would be expected even with continued defects in esophageal motility. Our data suggest that targeted intervention in dogs with LES-AS may provide substantial clinical benefit in this patient population, providing hope for dogs that are refractory to traditional medical management.(208)

Megaesophagus is characterized by diffuse dilation of the esophagus with decreased peristalsis. Unless, and sometimes even if, an underlying cause can be identified, treatment is largely supportive and carries a guarded to poor prognosis. Reported median survival times range from 1 to 3 months with an overall fatality rate of $74 \% .(172,174)$ Unfortunately, the majority of cases of ME are idiopathic with no clear understanding of an underlying pathologic process. (172) In humans, esophageal motility 
disorders are better classified allowing for identification of patients with conditions that may benefit from targeted intervention.(190) Achalasia, a primary esophageal motility disorder in people, results from a selective loss of inhibitory myenteric neurons leading to a failure of the LES to relax in response to pharyngeal swallowing and impaired esophageal peristalsis.(181) It represents a rare cause of ME that responds to targeted intervention and is considered distinct from conditions that cause esophageal hypomotility without functional LES obstruction. This condition has been suspected in dogs, with a few case reports over the last 4 decades and most presumptive diagnoses being made without manometry or dynamic imaging studies. $(182,207,209-213)$ The lack of recognition of this syndrome in dogs may in part be a consequence of limitations in available diagnostic tests. High resolution manometry is considered the gold standard for diagnosis of LES achalasia in people. Unfortunately, this modality is not routinely performed in veterinary medicine because of limited availability, high cost, need for operator expertise, and patient compliance. $(182,194)$ However, before the adoption of the HRM in people, contrast swallow studies were used to diagnose LES achalasia and although only moderately sensitive, were considered highly specific for this condition.(188, 199, 214) Few manometric studies in dogs with ME have been performed, and these did not identify LES achalasia to the extent documented in our study. $(194,215)$ The response to treatment in our patient population makes misdiagnosis unlikely, and this discrepancy between our study and previous studies may reflect limitations in available diagnostic tests at the time those studies were performed. Alternatively, it may highlight the point that this condition reflects subpopulations of dogs and not all dogs with ME, making accurate diagnosis critically important. 
Videofluoroscopic swallow studies, have long been considered the gold standard for evaluation of dysphagia in veterinary medicine, but because VFSS traditionally have been performed with dogs in lateral recumbency, and often by force-feeding, the risk of aspiration made such studies relatively contraindicated in dogs with ME, a diagnosis that could be made by routine thoracic radiography. However, thoracic radiography provides essentially no information regarding esophageal motility, which that has previously limited our understanding of the pathogenesis of the disease in dogs with ME. Using a protocol that allows dogs to stand and free-feed, we documented that $61 \%$ of dogs with ME that would have been classified as "idiopathic" had underlying LES-AS. These findings contrast with those of a large study of 216 dysphagic dogs in which VFSS performed with dogs in in lateral recumbency failed to identify any dogs with functional LES obstruction and historical manometric studies that failed to identify functional LES obstruction.(196, 215) Furthermore, the use of unrestrained and free-feeding VFSS protocols decreases the risk of aspiration to no more than would be expected in the dogs at home. To date, no dog at our institution has developed aspiration pneumonia after these unrestrained free-feeding VFSS, including those with ME. Thus, expanding the population that can be evaluated safely by VFSS has allowed identification and characterization of dogs with LES-AS, permitting specific treatment.(148)

The primary goal in treating LES-AS is early patient identification and treatment of esophageal-gastric outflow obstruction. In people, this goal is achieved either by mechanical disruption of the LES by dilation or surgical myotomy or by lowering LES tone. Sildenafil has been used with variable effect in humans to lower LES tone.(216) A recent study indicated improved clinical signs in puppies with congenital ME treated with 
sildenafil (without documentation of functional LES-obstruction), perhaps supporting the role of increased LES tone in the pathogenesis of ME in dogs.(185) In people, treatment is dictated largely by clinical variables, patient risk factors, and LES achalasia subclassification.(186, 217) Sub-classifications for LES achalasia in people are based on HRM, and treatment responses have been shown to vary based on achalasia subtype.(186, 218) Although subtypes have been identified in dogs with LES-AS based on fluoroscopy (148), numbers of affected animals are insufficient to attempt treatment based on subclassification. Furthermore, because achalasia subtypes in people are established by HRM rather than fluoroscopy, direct comparisons between subtypes in humans and dogs are not possible. As such, a combination of mechanical dilation and BTA injections was selected to maximize the likelihood of a response, and any dog with a positive response then subsequently was offered surgical intervention as a more lasting treatment.

In this study, treatment for LES-AS resulted in significant clinical and VFSS improvement after therapy targeting LES functional obstruction. Clients perceived clinical improvement in $100 \%$ of dogs by $2-3$ weeks after mechanical dilation and BTA injections. This finding is crucial, considering the degree to which perception of quality of life impacts treatment decisions in veterinary patients.(219) Although placebo effect may have played some role, this perceived clinical improvement is supported by pre- and post- treatment BW, BCS, frequency of regurgitation, and extent of gastric filling. All of the aforementioned metrics were significantly improved from baseline making a substantial placebo effect unlikely. Videofluoroscopic swallow studies documented improvement in passage of food from the esophagus to the stomach in the majority of dogs despite the persistence of ME and abnormal esophageal motility. This finding 
suggests that clinical improvement is secondary to relieving the functional LES obstruction, and supports the role of functional LES obstruction in the pathogenesis of ME in a subpopulation of dogs. The persistence of ME and esophageal dysmotility in these patients may reflect the short time frame between treatment and evaluation. Alternatively, irreversible structural changes may have occurred secondary to chronic dilatation. As such, clients should be informed that complete resolution of ME, esophageal dysmotility or both may not occur with targeted treatment but, this does not diminish the importance of the often dramatic clinical improvement. Two of 14 dogs did not show improved gastric filling after mechanical dilation and BTA injection despite improvement of other clinical variables. This finding has also been reported in people with achalasia following treatment, and in 1 study was predictive of long term treatment failure. (220) More study is needed before conclusions regarding this finding can be made in dogs with LES-AS.

Despite strongly positive responses to mechanical dilation with BTA injections, the relatively short duration of effect precludes their use as definitive (permanent) therapy for LES-AS. The increased risk of aspiration under general anesthesia makes multiple repeated endoscopic treatments less appealing. As such, mechanical dilation with BTA may be best reserved as a test for definitive surgical intervention, for temporary treatment for patients considered too high risk for myotomy (e.g. poor wound healing due to malnutrition), or as a temporary measure before surgery, particularly in young dogs before reaching skeletal maturity.

Although more data is needed, our study suggests that surgical intervention may provide sustained clinical improvement in dogs with LES-AS and that mechanical 
dilation and BTA injections may help identify patients that may benefit from surgical intervention. Interestingly, 2 dogs showed delayed ( $>6$ months later) evidence of decreased esophageal diameter and improved esophageal motility after surgical intervention compared to their initial VFSS evaluation. This observation is supported by findings in the human medical literature that suggest that esophageal pathology is secondary to increased LES tone, and treatment may result in a return to peristalsis in some patients without permanent damage. $(221,222)$

The age range of dogs with LES-AS was wide and inclusive of dogs with both congenital and acquired ME. Surgical intervention cannot be recommended until dogs reach skeletal maturity because gastric motility may be impacted by surgery, and final position of anatomic structures cannot be predicted in a growing animal. For these dogs, repeated treatment with mechanical dilation and BTA merits further evaluation. In people, mechanical dilation of the LES and injections of BTA can be repeated but submucosal fibrosis may occur with repeated treatment, potentially complicating future surgical intervention. $(223,224)$

Overall treatment complications in people with achalasia are approximately $6.3 \%$ with a peri-procedural mortality of $0.1 \%$. The most commonly reported complications after mechanical dilation and BTA injections in people include chest pain and mild heartburn, managed by antacids.(225, 226) Of note, chest pain and heartburn cannot be specifically detected in dogs. More serious complications in people including mediastinitis, allergic reactions to BTA and LES perforation are rare.(226) Complications after mechanical dilation with BTA in dogs included aspiration pneumonia and a Type IV hiatal hernia. Review of medical records could not reveal a potential cause of the 
complications in the dog with the hiatal hernia, but concurrent diffuse gastrointestinal dysmotility should be considered.

In people, LES mechanical dilation and BTA injections are performed as independent interventions. $(202,204,218)$ However, despite combining these 2 procedures in dogs, the duration of effect was considerably shorter than has been reported in people for either procedure alone. $(200,202,205,227)$ Dosing of BTA for achalasia is variable in people but ranges between 20-100 units.(228) A total of $32 \mathrm{U}$ (4 U/site) was selected based the smaller size of dogs compared with humans and to minimize the risk of complications associated with excessive administration.(225) Although doses were uniform for all dogs regardless of size, and the study was not powered to detect a dosedependent response. Additional studies would be needed to determine if higher doses result in a longer duration of effect.(229) Another explanation for the shorter duration of efficacy in dogs may involve anatomic differences between the canine and human esophagus. The entire canine esophagus is comprised of skeletal muscle compared to humans where the distal two-thirds of the esophagus is comprised of smooth muscle. Botulinum toxin A acts by interacting with several proteins including synaptosomalassociated protein (SNAP) 25 in the nerve terminal to prevent vesicle fusion and inhibiting the release of acetylcholine.(230) Differences in regional expression of SNAP25 in the esophagus have been reported in other species and could account for differences in treatment response.(231) Upregulation of SNAP-25 mRNA also has been identified in rats after BTA injections into skeletal muscle. (232) This finding suggests a possible role for SNAP-25 in functional muscle recovery and could account for variable responses if species differences are documented. Alternatively, differences in collagen composition, 
due either to species variation or extent of fibrosis at the time of diagnosis, may impact local diffusion of BTA and subsequent response to treatment.

Anatomic differences also may contribute to a shortened response to mechanical dilation. The purpose of mechanical dilation is to weaken the LES by tearing muscle fibers.(204) Differences in collagen versus muscle content may have made the extent of radial pressure applied by pneumatic dilation insufficient to achieve a sustained treatment response. Given the sustained treatment response seen after surgical myotomy, a greater extent of LES disruption may be needed. Mechanical dilation in adults is most commonly performed with balloons ranging from 3 to $4 \mathrm{~cm}$ in diameter. Smaller balloons are recommended in children, with balloons $>3.5 \mathrm{~cm}$ being reserved for children $>8$ years of age.(233) In our population, the balloon and bougienage diameter were selected based on patient size but ranged between $1-3 \mathrm{~cm}$. Selecting balloon size based on patient size was performed to decrease the risk of LES perforation. Blanching of the mucosa surrounding the LES was observed in all patients, suggesting resistance across the LES in response to balloon dilation and bougienage. Multiple dilation cycles were performed for each dog to maximize disruption of the LES. Multiple dilations with progressively increasing balloon diameter may result in a more sustained response, but may be associated with increased risk of LES perforation.

\section{Conclusions}

Dogs with LES-AS experienced marked clinical improvement after targeted intervention with mechanical dilation and BTA injections of the LES. Although the response was temporary, this finding establishes the role of functional LES obstruction in the pathogenesis of ME and esophageal dysphagia in dogs. Preliminary results from dogs 
with LES-AS following surgical myotomy, suggest that a positive response to mechanical dilation with BTA may identify dogs that could benefit from surgery, and that surgery may provide lasting clinical benefit despite persistence of ME. These interventions allow for often dramatic clinical improvement (improved quality of life, decrease episodes of regurgitation, weight gain, improved BCS) in a subpopulation of dogs with $\mathrm{ME}$ associated with LES-AS. 


\section{CHAPTER 5}

\section{DOCUMENTING SILENT REFLUX AND MICROASPIRATION EVENTS USING NUCLEAR SCINTIGRAPHY IN HEALTHY DOGS}

\section{Introduction}

Reflux is a known source of acute and chronic respiratory disease in people (2-6). Importantly, both reflux and microaspiration are also known to occur in healthy adult humans without obvious clinical consequence $(5,14)$. Clinically silent microaspiration highlights the importance of normative data before ascribing significance to reflux and/or microaspiration in the pathogenesis of canine respiratory disease. Repetitive microaspiration is a strong contributor to respiratory diseases in people $(82,234,235)$. Importantly, treatment of GER and extra-esophageal reflux (EER), reflux extending beyond the esophagus, reduces frequency of disease exacerbations and slows the rate of decline in lung function in people with asthma, chronic obstructive pulmonary disease, and idiopathic pulmonary fibrosis (2). As in people, a relationship between reflux, microaspiration, and respiratory disease is suspected in dogs based on a few clinical studies, case reports, and studies in animal models $(12,17,79,98,236-238)$. However, the frequency of reflux and microaspiration in healthy dogs has not been clearly established. The prevalence of gastroesophageal reflux (GER) in healthy, free-feeding dogs was found to be $41 \%$ by videofluoroscopic swallow study (VFSS) (239). However, this technique lacks the sensitivity to detect reflux associated with microaspiration. The ability to document reflux and microaspiration in dogs with respiratory disease may open doors for novel avenues of therapeutic intervention. As such, characterizing reflux and aspiration in normal dogs, and subsequently in dogs with aspiration associated respiratory 
disease (AARS), is an area of significant clinical relevance.

Current diagnostic methods for reflux and aspiration in dogs lack sensitivity and specificity due to intermittent reflux events, variable client vigilance, small volumes of refluxate, and the presence of non-acidic refluxate, which may account for as many as $90 \%$ of events in people $(19,112,113,117,121)$. Nuclear scintigraphy has potential to address many of these limitations and has been used successfully to detect reflux and aspiration people (127-131). For example, this technique has been used to safely evaluate pulmonary aspiration in medically fragile human infants $(127,130)$. In dogs this technique is postulated to be able to detect reflux events missed by owner observation and that may be missed by VFSS due to small volume. Scintigraphy may also detect nonacidic reflux events, broaden effective time of data collection by looking at additive radio-nuclide activity in several anatomic regions, and help establish the normative data necessary to determine the significance of positive results obtained in clinical patients (127-131). Nuclear scintigraphy may therefore represent a novel and sensitive means to help in the diagnosis of elusive reflux and AARS in dogs.

The objectives of this study are to characterize and determine the prevalence of reflux and aspiration events in healthy mesocephalic dogs and to obtain clinically relevant normative data to allow further exploration of nuclear scintigraphy as a diagnostic tool for dogs with suspected reflux and AARS. We hypothesize that healthy dogs will commonly have GER and a proportion of these dogs will have EER with silent aspiration into their respiratory tract.

\section{Materials and Methods}

\section{$\underline{\text { Animals }}$}


Twelve clinically healthy, adult, mesocephalic companion dogs were enrolled with informed consent (University of Missouri ACUC \#9871). Dogs were determined to be healthy based on normal physical examinations and the absence of clinical respiratory and GI disease within the preceding 6 months. Brachycephalic breeds and dogs receiving prokinetic medications were excluded.

\section{$\underline{\text { Reflux Scintigraphy }}$}

After a 12-hour fast, dogs were free-fed a standardized meal impregnated with (111 MBq) colloidal ${ }^{99} \mathrm{~m}$-technetium phytate $\left({ }^{99} \mathrm{TcP}\right)$ (Mid-America Isotopes, Ashland $\mathrm{MO})$. Each meal was followed by water $(10-20 \mathrm{~mL})$ to ensure clearance of radioactive material from the pharynx and esophagus. During data collection, non-invasive temporary markers were placed at the level of the mandible and stomach to ensure regions of interest (ROI) were maintained in the area of detection. Images were collected using a gamma camera with $140 \mathrm{keV}$ parallel hole collimator (Equistand, Middlesex, NJ) with Mirage computer system (Medical Imaging technologies, Akron, $\mathrm{OH}$ ) at 2 seconds/frame $(240,241)$. Dynamic studies were collected over 5 minutes in left-lateral and dorsal recumbancy $(\mathrm{n}=6)$ or in dorsal recumbancy alone $(\mathrm{n}=6)$. Data were collected 5- and 30-min post-ingestion of ${ }^{99} \mathrm{TcP}$. Static studies were collected for all 12 dogs in dorsal recumbancy in order to visualize both lung fields (right and left). Static data were collected over 2 minutes at 2 - and 18 -hours post ingestion of ${ }^{99} \mathrm{TcP}$. No abdominal pressure was applied. Dogs were housed in accordance with University of Missouri Environmental Health and Safety regulations between scans. All studies were performed without anesthesia or sedation.

\section{$\underline{\text { Data analysis }}$}


Data analysis was performed using 3DSlicer (version 4.10.1) and Fiji (ImageJ)(242) analysis software. For dynamic and static studies performed in dorsal recumbancy, ROI were drawn over the pharynx; proximal, middle, and distal esophagus; stomach; and right and left lung fields. (Figure 13a) For dynamic studies performed in left lateral recumbancy, lung ROI were not evaluated due to summation of the right and left lung fields. (Figure 13b) For dynamic studies, time-activity-curves (TAC), maximal reflux margination, volume, frequency, and duration were evaluated for each ROI (241). Reflux events (displayed on TAC) were defined as counts $\geq 200 \%$ background activity with a concurrent decrease in gastric counts (130). (Figure 14) Reflux TAC were subcategorized as rising (i.e. repeated reflux events with failure of clearance), flat (i.e. no reflux or reflux events with return to baseline between events) or falling (i.e. reflux with delayed clearance) (130). (Figure 15) Dogs with rising TAC were determined to have cumulative reflux events even if an exact number could not be quantified provided cumulative counts exceeded $200 \%$ of background. Maximal reflux margination was recorded as the maximal distance traveled (i.e. most distal ROI from the stomach) by reflux for each dog. Reflux volume was calculated as previously described (243). Reflux volume was depicted as the percent of gastric counts refluxed during each event. Reflux frequency was calculated as the number of discrete events occurring over the 5-minute collection period. Reflux duration was determined by the number of seconds counts remained $\geq 200 \%$ background. Static studies were used to confirm complete clearance of reflux after feeding (i.e. baseline), persistent ROI contamination and aspiration. Aspiration was defined as counts $\geq 200 \%$ baseline though a concurrent a drop in gastric counts were not considered necessary for diagnosis (130). 


\section{$\underline{\text { Statistical evaluation }}$}

Statistical analysis was performed using SigmaPlot (version 14.0) data analysis software. Descriptive statistics were applied where appropriate. Between group comparisons were made using a One-Way ANOVA on Ranks or Wilcoxon signed-rank test with a $p \leq 0.05$ significance level. Post-hoc analysis (Dunn's analysis for multiple comparisons) was performed where appropriate.

\section{Results}

\section{$\underline{\text { Animals }}$}

Twelve healthy companion dogs were enrolled with informed consent. Breeds represented included mixed breed $(\mathrm{n}=3)$, beagle $\operatorname{dog}(\mathrm{n}=2)$, Labrador retriever $(\mathrm{n}=2)$, and one each for miniature Dachshund, Jack Russel terrier, Brittany spaniel, Feist terrier, and West Highland white terrier. Seven dogs were castrated males and 5 were spayed females. Ages ranged from 3-13 years with a median (IQR) age of 9.5 years (6-10.25 years). Weights ranged from 7.1-26.0 kg with a median (IQR) weight of 9.6 kg (8.2-20.4 kg). Body condition score (9-point scale) ranged from 4 to 6 with a median (IQR) BCS of 5 $(5-5)$.

\section{$\underline{\text { Scintigraphy }}$}

Baseline scans demonstrated complete clearance of ${ }^{99} \mathrm{TcP}$ from the pharynx and esophagus at the start of the study. Reflux events were detected in all 12 dogs using reflux scintigraphy. A total of 144 TAC curves were further characterized as rising $(\mathrm{n}=27)$, flat $(\mathrm{n}=105)$, and falling $(\mathrm{n}=12)$. No significant differences were detected for demographic data (age, weight, or BCS), recumbancy (left lateral or dorsal), collection time (5 min or $30 \mathrm{~min}$ ), or ROI for TAC subcategory, maximal reflux extension, reflux 
frequency (events $/ 5$ minutes), or reflux duration (sec) ( $p>0.05$ for all). As such, groups were combined for further evaluation. Thirty-three discrete esophageal and 6 pharyngeal reflux events were identified. Pharyngeal reflux was identified in 5/12 dogs. Reflux marginated maximally to the pharynx (5/12 dogs), proximal esophagus (2/12 dogs), middle esophagus (3/12 dogs), and distal esophagus ( $2 / 12$ dogs). The total number of discrete reflux events identified within the esophageal ROI were 4/33, 6/33, and 23/33 for the proximal, middle, and distal esophagus respectively. The median (IQR) reflux volumes for esophageal and pharyngeal ROI are displayed in Table 1. Distal esophageal reflux volume was significantly greater than for pharyngeal reflux volume $(p<0.001)$. Reflux events detectable in the middle and proximal esophagus were not significantly greater in volume than pharyngeal reflux $(p>0.05)$. Median (IQR) frequency of reflux was 2 events/5 minutes (1-3.25 events/5 minutes). Median (IQR) duration (sec) of reflux was 6 seconds (4-9 seconds). Static scans showed no evidence of persistent contamination (counts $\geq 200 \%$ of background) within the pharyngeal or esophageal ROI for any dog regardless of timepoint (2hrs or 18hrs). Likewise, lung ROI showed no evidence of pulmonary aspiration for any healthy dog regardless of time-point (2hrs or 18hrs).

\section{Discussion}

In this pilot study, reflux scintigraphy was used to successfully identify and characterize reflux events in clinically healthy mesocephalic dogs. Reflux (GER and EER) but not pulmonary aspiration was a common finding in healthy dogs. This provides important normative data allowing further investigation into the role of reflux in the pathogenesis of respiratory disease. Investigating the relationship between reflux and 
aspiration is important as this may ultimately open new avenues of therapeutic intervention in affected dogs with a variety of respiratory disorders. Furthermore, since dogs and humans both have clinical manifestations of GER and EER, using a One Health approach will increase the opportunity for bidirectional advancement in research related to aspiration-associated respiratory syndromes (AARS).

People may have both reflux and aspirate without apparent clinical consequence due to the presence of functional protective and clearance mechanisms $(5,14,29)$. Interestingly reflux is also associated with the pathogenesis and progression of respiratory disease in human medicine with a prevalence of $50 \%$ in patients with chronic cough, asthma, COPD and pulmonary fibrosis (2-6). Therefore, the development of pathology is likely dictated by frequency, margination, volume, duration and content of reflux and aspiration rather than the presence or absence of such events. As such, data documenting the prevalence of reflux and aspiration in normal dogs and objective characterization of these events is necessary prior to ascribing pathologic significance to reflux and aspiration in clinically affected patients.

Airway-associated reflux can be subcategorized into GER and EER. Extraesophageal reflux is considered a supra-esophageal manifestation of GER with a demonstrated correlation between the severity of GER and prevalence of EER (244). Extra-esophageal reflux also increases risk of macro- and micro-aspiration, laryngeal dysfunction and exacerbation of pulmonary pathology $(244,245)$. Importantly, treatment of GER and EER has been demonstrated to reduce the frequency of disease exacerbations and slow the rate of decline in lung function in patients with airway and pulmonary parenchymal disease (2). A study in brachycephalic dogs likewise demonstrated that 
treatment for reflux prior to brachycephalic airway surgery resulted in decreased postoperative complications (17). Treatment for GER and EER may therefore reflect a potential avenue of therapeutic intervention for dogs with AARS. Unfortunately, studies evaluating the relationship between GER, EER, aspiration and the development of respiratory disease are conspicuously absent in the veterinary literature. This due in part because diagnostics capable of identifying dogs with naturally occurring GER, EER, and microaspiration are lacking.

Reflux scintigraphy with ${ }^{99} \mathrm{TcP}$ is considered a safe and sensitive means to detect reflux and microaspiration in people (127-130). Because ${ }^{99} \mathrm{TcP}$ is not absorbed systemically, the presence of increased counts in the esophagus and pharynx, as well as the lungs after initial clearance into the stomach are diagnostic for reflux and aspiration, respectively. In adult people, cough and laryngospasm were strongly correlated with positive reflux/aspiration scintigraphy (130). Further, patients who were positive for reflux via scintigraphy had symptomatic response to surgical treatment: $90 \%$ of cases had resolution of clinical symptoms suggesting a high predictive value for scintigraphy findings (130).

In this study, reflux was identified in $100 \%$ of dogs. This is greater than the $41 \%$ reported in healthy dogs evaluated by VFSS (239). This may be attributed to the increased sensitivity of scintigraphy compared to fluoroscopy for small volume reflux as well as the longer collection period of scintigraphy compared to VFSS (246). Increased intragastric pressure during recumbancy may also be contributory and clinically relevant (i.e., it would be common for a dog to lay down after eating). Decreased numbers of transient lower esophageal sphincter relaxations have been demonstrated in dogs and 
people in supine compared to upright posture (247-249). As such, increased frequency of transient lower esophageal sphincter relaxations, due to dorsal and lateral recumbancy are unlikely to contribute to the increased number of dogs with reflux observed in this study. The majority of dogs in this study had an ideal or near ideal body condition score which was similar to the population of dogs evaluated in the aforementioned VFSS study (239). As such, body condition is unlikely to contribute to the increased prevalence of reflux noted with scintigraphy. The presence of detectable reflux in $100 \%$ of healthy dogs evaluated by reflux scintigraphy highlights the need for additional parameters to discriminate between healthy dogs and those with pathologic reflux.

The use of time activity curves (TAC) allows for quantification of repeat reflux events and are routinely evaluated in reflux scintigraphy studies in people $(130,250)$. The presence of a rising TAC is considered supportive of cumulative reflux events though individual reflux events cannot necessarily be visualized due to superimposition. Individual reflux events were determined by counts $\geq 200 \%$ with concurrent drops in gastric counts (Figure 2). However, reflux events were not always tracible to their maximal extent in each ROI (e.g. reflux events detectable in ROI E1 and E3 but not E2). This was likely attributable to rapid transit times compared to sampling rate. Similarly discontinuous tracings may be seen in people with reflux detected by impedance manometry as well as scintigraphy $(251,252)$. Such discontinuous tracings precluded correlation of total reflux volume with extent of margination. As such, reflux volume was calculated according the volume calculated within each ROI. A higher frame rate may improve detection of individual reflux events and additional studies in dogs are needed to establish a correlation between severity of GER and the presence of EER as is seen in 
people $(240,241,244)$. Despite this limitation, the frame rate selected for this study is otherwise considered sufficient. Sampling rates 1-6 seconds/frame are used for pediatric gastric scintigraphy studies. Further, esophageal $\mathrm{pH}$ studies have demonstrated that increased sampling rate did not correlate with increased acid exposure time, and it has been postulated that brief reflux events are less likely to be clinically relevant than prolonged esophageal contact with refluxate $(131,241,253,254)$. As a rising TAC curve is indicative of esophageal exposure to gastric contents, the sampling rate in this study is not considered a significant limitation. In this study all TAC subcategories (rising, flat and falling) were represented. The presence of EER in this study was not associated with a rising TAC as has been demonstrated in some studies in people though the influence of type 2 error cannot be ruled out (130). Studies in clinically affected dogs are needed to determine the significance of flat and falling TAC.

Pharyngeal reflux (i.e., EER) was identified in nearly $42 \%(5 / 12)$ of dogs representing approximately $15 \%$ of total discrete reflux events detected. This mirrors findings in people where EER events were detectable in 10-30\% of asymptomatic adults evaluated by impedance manometry (255). Extra-esophageal reflux is clinically important as laryngeal structures are susceptible to damage by acid and digestive enzymes (256-258). In the esophagus, up to 40 reflux episodes per day is considered normal in people; however, as few as 3 reflux episodes reaching the larynx are sufficient to cause detectable damage (258). In dogs without EER, maximal esophageal margination for the proximal, middle, and distal esophagus was approximately $16.7 \%$ (2/12), $25 \%(3 / 12)$, and $16.7 \%(2 / 12)$ accounting for $70 \%(23 / 33), 18 \%(6 / 33)$, and $12 \%$ (4/33) of detected individual esophageal reflux events respectively. This is a departure 
from VFSS where the majority of reflux events appeared to be contained within the distal esophagus (239). In healthy adults, proximal esophageal reflux was identified in $34 \%$ of upright reflux events by esophageal impedance $\mathrm{pH}$ monitoring. Additional studies evaluating the reflux margination in people with gastroesophageal reflux disease (GERD) and extra-esophageal reflux disease (EERD) suggest that symptomatic patients have a higher median proximal margination of reflux events, larger reflux volumes, and longer reflux durations than healthy controls (259).

The volume of refluxate is considered important as it reflects the total amount of potentially damaging substances. Absolute quantification of reflux volumes is technically challenging but may be performed by calculating the percent of gastric counts that are detected in the esophagus during reflux events. Large volume reflux events ( $>4 \%)$ were occasionally detected in this population. The volume of reflux in the distal esophagus was considerably greater than in the other esophageal and pharyngeal ROI suggesting decreasing volumes of refluxate with increased margination from the stomach. Frequency and duration of reflux events were able to be characterized by reflux scintigraphy suggesting utility in clinical patients, however controlled studies comparing objective parameters in healthy and clinically affected dogs are needed.

Interestingly, aspiration was not identified in any healthy dog, which is in opposition to the human literature. Species differences may account for this variation, however, type 2 error cannot be ruled out and further studies are needed. Lack of sensitivity for detecting aspiration is considered unlikely. Computer acquisition of data demonstrated that as little as $0.1 \mathrm{MBq}$ of activity aspirated into the lungs can be detected by the gamma camera (260). This suggests that microaspiration is likely to be detected 
where present.

Limitations of this study include small sample size. Further, not all dogs were evaluated both in left-lateral and dorsal recumbancy. Importantly, no differences were identified with recumbency, which is important as dorsal recumbency allows independent visualization of the right and left lung fields. Ultimately these do not alter the conclusion that reflux scintigraphy represents a viable adjunctive diagnostic for dogs with reflux and AARS especially when repetitive microaspiration is suspected. Finally, only mesocephalic dogs were evaluated in this study. Given the presumed predisposition of brachycephalic for reflux and AARS, additional studies specifically evaluating this population is needed.

\section{Conclusions}

Gastric reflux to the level of the esophagus and pharynx, but not pulmonary aspiration, were common findings in healthy mesocephalic dogs evaluated by nuclear scintigraphy. The results of this study support further exploration of reflux scintigraphy as an adjunctive diagnostic in dogs with aerodigestive disorders. The normative data presented here may help in the interpretation of future studies in dogs with suspected AARS. 


\section{CHAPTER 6}

\section{PROTEOMIC BIOMARKERS IN REGURGITATION, VOMITING, AND COUGH: PROTEOMIC CHARACTERIZATION OF CANINE GASTRIC FLUID BY LIQUID CHROMATOGRAPHY MASS SPECTROMETRY (LC-MS)}

\section{Introduction}

Biomarkers diagnose, prognosticate, monitor, and identify populations at risk for disease. Development and implementation of biomarkers has been making an increasingly large impact in companion animals. Biomarkers have the potential to advance understanding of disease pathogenesis, allow objective investigation of efficacy of novel therapeutics, and advance translational research through animal models of human disease $(132,261-264)$.

Reflux of gastrointestinal contents is a source of acute and chronic pulmonary disease with a prevalence of $50 \%$ in humans with chronic cough, asthma, chronic obstructive pulmonary disease and idiopathic pulmonary fibrosis (2-6). Reflux is subcategorized into gastroesophageal reflux (GER) and extraesophageal reflux (EER). Gastroesophageal reflux refers to reflux of gastric contents into the esophagus while EER refers to refluxate reaching structures beyond the esophagus including the oro-and nasopharynx, larynx, and airways (245). Severity of GER and prevalence of EER are correlated (244). In people, EER increases risk of macro- and microaspiration, laryngeal dysfunction and exacerbation of pulmonary pathology (244, 245). Importantly, treatment of EER in people reduces frequency of disease exacerbations and slows rates of decline in lung function (2). This makes identifying patients with reflux-associated respiratory disease critically important. 
Reflux and aspiration are implicated in canine respiratory disorders including rhinitis, laryngeal dysfunction, and upper/lower airway and pulmonary parenchymal diseases $(12,79,98)$. Despite the implied association between reflux, aspiration, and respiratory disease, a non-invasive widely available means of detecting EER in dogs is needed. Human gastric fluid (GF) has been extensively characterized allowing for identification of reflux biomarkers outside the gut (265-267). Gastric pepsin (pepsin A), a component of refluxate identified in humans with EER, is a biomarker in oral and respiratory secretions with improved sensitivity (100\%) over ambulatory esophageal $\mathrm{pH}$ monitoring $(63 \%)(9,115,266-268)$. Though proteomic evaluation has been performed for several biological fluids in dogs, $(264,269)$ characterization of the canine GF proteome has not been performed previously.

The objectives of this pilot study were 2-fold. The first was to perform a comprehensive characterization of the protein composition of canine GF using liquid chromatography mass spectrometry (LC-MS) and to compare the GF proteomic profile to that of the oropharynx in healthy, vomiting/regurgitating, and coughing dogs to evaluate for potential biomarkers of EER. The second objective was to evaluate potential biomarkers for stability by evaluating samples after storage at $20^{\circ} \mathrm{C}$ for $\leq 2$ weeks and 6 months. We hypothesized that using high coverage proteome analysis we could identify stable proteins relevant as potential biomarkers of canine EERD.

\section{Materials and Methods}

\section{$\underline{\text { Animals }}$}

Twenty-three companion dogs presenting to the University of Missouri Veterinary 
Health Center were prospectively enrolled with informed owner consent (University of Missouri ACUC 240). Residual canine GF was collected directly via nasogastric or orogastric tubes in patients for whom these interventions were deemed medically necessary by the attending clinician. Oropharyngeal swabs were collected from healthy dogs, dogs with a history of regurgitation/vomiting, and coughing dogs. Heathy dogs had an unremarkable physical examination and no clinical evidence of gastrointestinal (GI) or respiratory disease within the preceding 6 months. Regurgitating/vomiting dogs were enrolled if they had a documented episode of regurgitation or vomiting within 12 hours of sample collection. Coughing dogs were enrolled if they had a history of cough for greater than 2 weeks without clinical evidence of gastrointestinal disease (e.g., vomiting, regurgitation). Exclusion criteria included dogs with mixed clinical evidence of respiratory and GI disease and dogs that were currently receiving pro-kinetics, antibiotics, or probiotics at the time of sample collection.

\section{$\underline{\text { Sample Collection }}$}

Two $\mathrm{mL}$ of GF were collected via nasogastric or orogastric tube and placed in a sterile red top tube. Oropharyngeal swabs, collected by vigorously rubbing this region in each gently restrained dog meeting our inclusion criteria, were placed in $2 \mathrm{~mL}$ of sterile saline in a sterile red top tube as above. The samples were gently vortexed, and even aliquots distributed into 2 polypropylene conical bottom Eppendorf tubes (Fischer Scientific, Chicago, IL). Paired samples were frozen at $-20^{\circ} \mathrm{C} ; 1$ aliquot was evaluated within 2 weeks and the other was evaluated 6 months after sample collection.

\section{$\underline{\text { Proteome Analysis }}$}

Protein was extracted using 4 volumes of ice-cold acetone. Following washing 
with $80 \%$ acetone (in water) the pellet was re-suspended with $6 \mathrm{M}$ urea, $2 \mathrm{M}$ thiourea, and $100 \mathrm{mM}$ ammonium bicarbonate. Protein was quantified using the EZQ assay according to the manufacturer's instructions (Invitrogen/Life technologies). After quantification, protein amount normalized across all samples by dilution in the urea buffer, and $25 \mu \mathrm{g}$ protein from each sample were digested with trypsin. The resulting peptides were desalted using C18 pipette tips (Peirce/Thermo), lyophilized, and resuspended in $25 \mu 1$ of acetonitrile/formic acid (0.1\%). Ten ug of the re-suspended peptides was loaded on a C8 trap column (Thermo PepMap 100, 5cm x 300um) and then separated using a $400 \mathrm{~nL} / \mathrm{min} 70 \mathrm{~min}$ gradient on a self-packed C18 column (75 um x 20 $\mathrm{cm} \times 1.7$ um particles - Waters BEH C18) at $50{ }^{\circ} \mathrm{C}$ in the CaptiveSpray nanospray source. The mobile phase consisted of $0.1 \%$ formic acid in water (A) and $0.1 \%$ formic acid in acetonitrile (B) and the LC gradient was for B: Initial 2\%, followed by 26 min ramp to $17 \%, 17-25 \%$ over $36 \mathrm{~min}, 25-37 \%$ over $15 \mathrm{~min}$, gradient of $37-80 \%$ over 6 min, hold at $80 \%$ for 7 min with a total run time of $90 \mathrm{~min} . \mathrm{MS}+\mathrm{MS} / \mathrm{MS}$ data were acquired on a Proxeon Easy nLC system attached to an TQ Orbitrap mass spectrometer or Bruker timsTOF-PRO using the PASEF(1) method over the 90min gradient. Capillary voltage was set to $1600 \mathrm{~V}$, tims-on, PASEF-on (10 PASEF frames, overlap of 5; i.e. between MS acquisition), 100-1700 m/z mass range. Cycle time for $1 \mathrm{MS}$ and 15 PASEF $=1.8 \mathrm{sec}$ (approximately $120 \mathrm{MS} / \mathrm{MS}$ acquired per cycle). Repeated acquisition: threshold $4 \mathrm{X}$ within 0.4 min (if same peptide precursor is observed at a $4 \mathrm{X}$ intensity within the RT threshold, re-acquire MSMS). Active exclusion: release after $1 \mathrm{~min}$ (exclude from MS/MS the same peptide mass, notwithstanding the criteria above, and release after 1 min of elution). MS data were collected over an m/z range of 100 to 1700 . 
During MS/MS data collection, each trapped ion mobility spectrometry (tims) cycle included $1 \mathrm{MS}+$ an average of 10 parallel Accumulation Serial Fragmentation (PASEF) MS/MS scans.

\section{Samples stored at $-20^{\circ} \mathrm{C}$}

During the 6-month storage period, the MU proteomics core underwent a systems upgrade. Samples were processed as previously described; however, peptides were acquired using the Bruker timsTOF pro (Bruker Scientific Billerica, MA).

\section{$\underline{\text { Data Analysis }}$}

The acquired data were submitted to the PEAKS DB search engine (version $\mathrm{X}$, Bioinformatics Solutions Inc.) for peak picking and protein identification using NCBI database limited to dog sequences $(81,077$ entries, last update $3 / 1 / 2019)$ or cat sequences (45,259 entries; last update 5/1/2019). Database search parameters were as follows: parent mass tolerance: $50 \mathrm{ppm}$; fragment mass tolerance: $0.1 \mathrm{Da}$; precursor mass type: monoisotopic; enzyme: trypsin; max \# cleavages: 2; non-specific cleavage: 1 (consider peptides with one end that is tryptic); fixed modification: carbamidomethyl Cys; variable mods: Met oxidation and deamidation. FDR estimation enabled (a reversed decoy database is created and searched simultaneously). Differential abundance of proteins was determined using PEAKS LFQ (label-free quantitation) based on the MS1 peak integration approach with the following parameters: algorithm: PEAKS-Q; retention time tolerance: $1 \mathrm{~min}$; mass tolerance: $40 \mathrm{ppm}$. Data were then filtered for 1\% protein FDR with $\geq 1$ unique peptide and $\geq 2$-fold change. Spectral counting was used to determine differential protein abundance using the following criteria: data were then filtered for $1 \%$ protein FDR with $\geq 1$ unique peptide and then $>4$ spectral counts per protein (mean of 
replicates). Protein abundance is described using the normalized spectral abundance factor (NSAF). Biological function was determined for the 200 most abundant proteins per group (GF, normal, vomiting/regurgitation, coughing) as determined by NSAF. Biomarker candidates were evaluated according to their abundance in GF, tissue specificity, low or absent concentrations in normal dog OP, and increased concentrations in vomiting/regurgitating compared to normal dog OP. Candidate proteins were then assessed in coughing dogs to look for evidence of EERD.

\section{Statistical Analysis}

Statistical analysis was performed using Past (version 3.21) and SigmaPlot (version 14.0) data analysis software. Descriptive statistics were applied where appropriate. Differences in patient demographics between groups (GF, healthy, vomiting/regurgitation, and cough) were evaluated by Wilcoxon signed rank test with $p \leq$ 0.05 significance level. Principle component analysis (PCA) was performed for all proteins identified across groups. Differential abundance of proteins between groups based on pairwise comparisons were evaluated by Fischer Exact test with $p<0.0004$ considered significant. Coefficient of variation was calculated for each protein demonstrating differential abundance between groups. A one-way Analysis of Similarity (ANOSIM) using a Bray-Curtis similarity index was used to test for similarity between groups based on the biological function of detected proteins with $p \leq 0.001$ significance level. Normality was evaluated by Shapiro-Wilk test. Post-hoc analysis (Dunn's method or Bonferroni correction for multiple comparisons) was applied where appropriate. Due to differences in sensitivity between collection methods (TQ Orbitrap mass spectrometer at the early time point vs. Bruker timsTOF pro at the 6-month time point), exact 
comparisons before and after storage could not be performed. Candidate biomarkers identified in the initial run were therefore described as present or absent following 6 months of storage of $-20^{\circ} \mathrm{C}$.

\section{Results}

\section{$\underline{\text { Animals }}$}

Twenty-three companion dogs were prospectively enrolled with informed consent. Breeds represented included mixed breed $(n=5)$, Labrador retriever $(n=3)$, Yorkshire terrier $(\mathrm{n}=2)$, standard poodle $(\mathrm{n}=2)$, and one each for Australian shepherd, Welsh corgi, basset hound, Jack Russel terrier, beagle dogs, golden retriever, German Shepherd dog, Boston terrier, bull terrier, miniature poodle, and Catahoula leopard dog. Twelve dogs were spayed females, 7 were castrated males, 2 were intact males, and 2 were intact females. No significant differences were detected between groups (GF $(n=5)$, healthy $(\mathrm{n}=6)$, vomiting/regurgitation $(\mathrm{n}=7)$ and cough $(\mathrm{n}=5))$ for age, weight, or body condition score (BCS). These results were therefore grouped and displayed as range and median (IQR). Ages ranged from 6 months to 13 years with a median (IQR) age of 9 years (7-10 years). Weights ranged from $1.7-43 \mathrm{~kg}$ with a median (IQR) weight of 16.6 $\mathrm{kg}(9-29 \mathrm{~kg})$. Body condition score (9-point scale) ranged from 4 to 8 with a median (IQR) BCS of 5 (5-6).

\section{$\underline{\text { Proteomic analysis }}$}

From all samples, a total of 504 individual proteins were identified. Within group evaluation showed no significant differences for the total number of proteins identified. The median (IQR) number of proteins (per sample) per group were GF: 122 proteins (110-230), healthy: 259 proteins (203-209), vomiting/regurgitation: 231 proteins (203- 
240 ) and cough: 258 proteins (254-325). No significant differences between groups were found for the total number of proteins identified. Principal components analysis demonstrated overlap between GF, vomiting/regurgitation, and cough but not normal OP (Figure 16).

Three pairwise comparisons were performed to identify differentially abundant proteins between groups: 1) normal vs GF, 2) normal vs vomiting/regurgitation, 3) normal vs cough.

Normal vs GF: One hundred thirty proteins showed significant differential abundance $(p<0.0004)$. Seventy proteins were significantly greater in abundance in GF; 60 were significantly greater in OP swabs from normal dogs. Coefficients of variation between dogs ranged from 16.9- 244.9 for the differentially abundant proteins.

Normal vs Vomiting/Regurgitation: Twenty proteins showed significant differential abundance $(p<0.0004)$. Thirteen proteins were significantly greater in abundance in OP swabs from vomiting/regurgitating dogs; 7 were significantly greater in OP swabs from normal dogs. Coefficients of variation between dogs ranged from 45.5-264.6 for the differentially abundant proteins.

Normal vs Cough: Twenty-two proteins showed significant differential abundance $(p<0.0004)$. Twelve proteins were significantly greater in abundance in OP swabs from coughing dogs; ten were significantly greater in OP swabs from normal dogs. Coefficients of variation between dogs ranged from 41.7-244.9 for differentially abundant proteins.

For the biologic function of proteins, all groups were found to be dissimilar on ANOSIM with $p \leq 0.05$ (Figure 17). Metabolic and immune proteins were over- 
represented in GF compared to normal dog OP swabs $(\mathrm{p} \leq 0.05)$. Seventy-three percent of gastric metabolic proteins were pancreatic in origin which is over-represented compared to salivary (14\%), gastric $(10 \%)$ and intestinal $(3 \%)$ proteins $(p \leq 0.05)$. Pancreatic metabolic proteins were also increased in overall abundance compared to salivary, gastric and intestinal metabolic proteins by NSAF. Pepsin A was identified in GF from all dogs but was less abundant compared to other non-gastric metabolic proteins (Table 15). Metabolic proteins were likewise over-represented in OP swabs in vomiting/regurgitating dogs compared to healthy dogs ( $\mathrm{p}<0.05)$, as were proteins with immunologic function ( $p$ $<0.05)$. Coughing dogs had increased numbers of proteins involved in cellular differentiation and stimulus response compared to normal and regurgitating/vomiting dogs $(p<0.05)$. After 6 months of storage at $-20^{\circ} \mathrm{C}, 54 / 70,11 / 13$, and $8 / 12$ proteins demonstrating differential abundance compared to healthy dog OP in the first run were still detectable for GF, vomiting/regurgitation and coughing dogs respectively.

\section{Discussion}

In this pilot study, untargeted LC-MS was used to successfully characterize the canine GF proteome and identify several candidate biomarker proteins demonstrating differential abundance in health and disease in the dog. The proteome of gastric fluid showed statistically significant differences compared to healthy dog OP. This suggests the possible utility of protein biomarkers as screening tool for dogs with EERD and aspiration. Reflux and aspiration contribute significantly to the pathogenesis and progression of respiratory disease in people $(2,3,5,6,236)$. A similar relationship is suspected in veterinary patients, and although a readily available diagnostic test capable of detecting EERD and aspiration is lacking (17) a proteomics approach shows promise. 
Though further studies are needed, significant between-dog variability may suggest improved utility of biomarker panels over individual proteins. Detection of candidate biomarkers was possible after storage at -20 for 6 months which provides important preliminary data for those engaged in clinical research requiring sample banking.

Understanding the link between reflux and aspiration in clinical patients is critically important as people with reflux-associated respiratory disease have demonstrated reductions in disease exacerbation and decline in lung function following treatment targeting GERD and EERD (2). A similar targeted therapeutic approach may be beneficial in dogs with airway, interstitial and/or pulmonary parenchymal diseases. In experimental canine models, a link between respiratory disease, reflux and aspiration in dogs has been demonstrated with bronchoconstriction, laryngospasm, laryngeal paresis/paralysis, and microaspiration occurring in response to application of acid and digestive enzymes to the esophagus and larynx (13-16). In pet dogs, this association is less clear. In brachycephalic dogs, an animal model for obstructive sleep apnea in people, treatment for presumptive GERD/EERD significantly improved clinical signs of brachycephalic airway syndrome (BAS) and minimized post-surgical complications $(17,236,237)$. As such, treatment for EERD may also benefit other dogs with other naturally developing respiratory disorders in which reflux and repetitive microaspiration play a role in disease development, progression and exacerbation (12). Furthermore, similarities in anatomy, physiology and several pathologic disorders makes experimental and naturally occurring canine models integral to translational research in humans (270-273).

Identifying dogs with naturally occurring GERD and EERD poses a diagnostic challenge as reliable, inexpensive, minimally invasive diagnostic tests are lacking. In 
people, biomarkers have been effectively utilized as a part of a multimodal approach to diagnosing reflux and aspiration-associated respiratory syndromes (AARS) $(3,9,123$, 266). Though biomarkers have been utilized effectively for a number of diseases in dogs, investigation into biomarkers of reflux and aspiration have been rarely performed (98, $132,262,269,274)$. In this study, LC-MS was successfully used to generate a proteomic profile of canine GF to identify potential biomarker candidates. Principal component analysis demonstrated complete separation with a CI of $95 \%$ of the GF proteome and normal dog OP proteome suggesting that these two sites significantly different with respect to their protein composition. This is a key initial finding supporting in absence of reflux there are no gastric proteins in the healthy dog OP and sets the stage to identify biomarker gastric proteins in this location in disease states.

Statistically significant differential abundance for multiple proteins was identified between all groups compared to normal dogs. This finding suggests that the canine proteome differs not only by the site of collection (GF vs. OP (normal)) but also in health and disease (vomiting/regurgitation or cough vs. normal). Principal component analysis demonstrated overlap between GF, vomiting/regurgitating, and coughing proteomes. No overlap was identified between GF and normal dog OP. Though further studies are needed, this supports hypothesis that GF biomarkers may be identified in vomiting/regurgitating and coughing dogs. The overlap between vomiting/regurgitating, coughing and healthy dog OP likely reflect a shared site of collection. Despite this overlap, a number of proteins demonstrating statistically significant differential abundance were identified.

Gastric pepsin (pepsin A) is an abundant component of the human gastric 
refluxate which has been used as a biomarker to identify patients with EER and aspiration $(9,266-268,275)$. While pepsin A was identified in canine GF, it was low in abundance. Further, pepsin was not identified in OP swabs from any dog including those with documented regurgitation/vomiting within 12 hours of sample collection. It is possible that protein degradation may have reduced the abundance of this protein below our limit of detection. The low abundance of pepsin A in GF and lack of detection of pepsin A on OP swabs in vomiting/regurgitating dogs suggests this to be a less than ideal protein biomarker regardless of cause, and represents a significant departure from the human literature $(100,122,123,266,276)$. Interestingly, pancreatic proteins were highly abundant in GF suggesting that gastroduodenal reflux was a frequent occurrence in our population and supports a biomarker study which identified bile in bronchoalveolar lavage fluid from dogs with pulmonary fibrosis (98). In human gastric fluid pancreatic proteins are also identified, however the abundance is insufficient to overshadow pepsin A in gastric fluid (275). Salivary proteins were abundant in gastric fluid as well as in dogs with vomiting/regurgitation. The abundance of salivary proteins in GF likely reflects reflexive swallow of salivary secretions (277). Increased abundance in dogs with vomiting/regurgitation may reflect bathing of the $\mathrm{OP}$ with concentrated salivary secretions from the gastric fluid, ptyalism secondary to nausea, or as a result of the esophagosalivary reflex which increases salivary secretions to protect against damage to the esophagus by gastric acid/digestive enzymes (278).

While biomarker candidates based on our criteria were identified in dogs with vomiting/regurgitation, evidence of EER was not identified in any coughing dog. This likely reflect our small sample size (5 dogs with cough) and larger studies in respiratory 
patients are needed before drawing conclusions about the prevalence of EER in dogs. The presence of gastric proteins demonstrating increased abundance in OP swabs from vomiting/regurgitation compared to normal dogs opens doors for future biomarker validation studies in dogs with EERD and AARS. Important to future biomarker studies was that protein concentrations between dogs was extremely variable echoing similar findings in people, suggesting limited utility for individual protein biomarkers in favor of biomarkers panels (279-281).

Biological protein function was determined for the 200 most abundant proteins per group and statistical evaluation by ANOSIM demonstrated that OP swabs from normal dogs, vomiting/regurgitating, and coughing dogs were statistically dissimilar representing discrete functional proteomic profiles. This suggests that the differences in composition between groups may be enough to alter the functional resident proteome in health and disease. In this case, even though vomiting/regurgitating and coughing dogs demonstrated overlap according to PCA, differences in relative abundance were enough to change the functional proteomic profile between health and disease, and between 2 disease states (vomiting/regurgitating and coughing). In vomiting/regurgitating dogs, proteins with metabolic (i.e. enzymatic) functions were found in increased abundance compared to normal dogs. This may reflect a contribution from the GF proteome though the overall functional proteomic profiles were still considered dissimilar between these two groups. Though further studies are needed the functional proteome may reflect an additional means to identify dogs affected by GERD and EERD that is less affected by individual protein concentrations which vary significantly between dogs.

Sample banking is an important part of clinical research and human and 
veterinary medicine (282). Large scale biomarker validation trials reflect collaborative interdisciplinary efforts involving both scientists and clinicians with access to variable methods of cold storage (283). Banking at $-20^{\circ} \mathrm{C}$ was selected to reflect the most common means of sample banking available to general practitioners. Most proteins in vomiting/regurgitating and coughing dogs exhibiting differential abundance were present after 6 months of storage. However, due to unforeseen differences in instrumentation during the time of the current study, absolute degradation could not be determined. Additional studies evaluating the degradation of candidate biomarkers is recommended before making recommendations regarding long term sample storage.

\section{Conclusions}

Extra-esophageal reflux and aspiration represent a significant contributor to a number of acute and chronic respiratory diseases in people. Importantly, therapy targeting GER and EER may positively impact management of people with AARS. Proteomic characterization of GF and the OP in vomiting/regurgitating dogs identified potential candidate proteins for future studies investigating biomarker panels for EER and AARS. Unlike in people, pepsin was poorly abundant in gastric fluid and non-detectable in dogs with vomiting/regurgitation. Salivary and pancreatic proteins were abundant in GF and may reflect potential biomarkers for evaluation of patients with suspected EERD and AARS. Short-term sample storage resulted in detectable differences between groups, and those proteins could be identified after 6 months. 


\section{CHAPTER 7}

\section{DESCRIMINATION BETWEEN RESPIRATORY AND NON- RESPIRATORY SOUND WAVEFORMS IN DOGS USING ACOUSTIC WAVE RECORDINGS: AN OBJECTIVE METRIC OF COUGH}

\section{Introduction}

Cough is a critical defense mechanism of the respiratory system and provides an important metric for disease control. Chronic cough may lead to exacerbation of structural airway disease through perpetuation of inflammation. In addition, cough represents a significant source of frustration for both dog and client, and severe cough is associated with a perceived reduction in quality of life (284). Unfortunately, management of cough in veterinary medicine is often suboptimal and based on subjective response rather than objective evidence. As such, objective metrics of cough are needed.

The assessment of cough in veterinary medicine currently employs measures based on client surveys, visual analogue scale (VAS) scores, and other subjective measures of clinical scoring $(285,286)$. Owner stress associated with witnessing coughing episodes may lead to client overestimation of cough severity, while the episodic nature of cough and variable client vigilance may lead to underestimation. A study evaluating compliance and accuracy of self-reporting in human asthmatics found $23 \%$ of subjective client-recorded metrics being fabricated (287). These confounding factors, combined with the placebo effect, have the potential to alter the conclusions of treatment trials, which are critical for management recommendations of chronic respiratory disease. Current subjective metrics represent significant challenges and highlight the need for objective, independently recorded biometrics to aid in clinical case management as well 
as placebo-controlled clinical trials.

In people, cough generates high velocity, non-laminar airflow producing a characteristic auditory signal $(139,288)$. The resulting acoustic waveform can be detected and objectively evaluated $(139,288-291)$. Audio-based cough monitoring in human medicine is capable of providing reliable data on the number of coughs by examining acoustic waveforms with sensitivity and specificity of $85 \%$ and $91 \%$, respectively (139, $288,290,291)$. We hypothesized that healthy dogs would demonstrate characteristic and consistent respiratory waveforms which could be distinguished from other acoustic behaviors (AB). The objectives of this study were three-fold. The first was to develop a sound recording protocol to capture respiratory waveforms in healthy dogs. The second was to discriminate cough events from other $\mathrm{AB}$ based on objective acoustic parameters (AP). The final objective was to determine if a trained investigator could, when blinded to randomized data, correctly identify each AB.

\section{Materials and methods}

$\underline{\text { Dogs }}$

Healthy employee-owned companion dogs were enrolled with informed consent. Dogs were determined to be healthy based on clinical history and physical examination. Inclusion criteria included being greater than one year of age with no history of respiratory or gastrointestinal signs within the preceding 6 months. Patients with a prior history of tracheal stenting, laryngeal tie back surgery, and/or current treatment with antitussive medications were excluded. Age, sex, body weight, body condition score (BCS), and breed were recorded for each dog to evaluate for changes in $\mathrm{AB}$ based on patient demographics. 


\section{$\underline{\text { Acoustic behavior recordings }}$}

A laryngeal microphone (CTA Digital, Brooklyn, NY, USA) was used to record eight common AB: cough, chew, lick, bark, whine, drink, growl, and throat clear. Dogs were minimally restrained and maintained in a standing position during data collection. A recording microphone positioned over the larynx was connected to a laptop computer (Figure 18). Data were collected at a sampling rate of $44.1 \mathrm{kHz}$. The frequency response for the cardioid (unidirectional) microphone was between $20 \mathrm{~Hz}$ and $20 \mathrm{kHz}$. The recordings were analyzed using Raven Pro (C) sound analysis software (version 1.4, Cornell Ornithology Laboratory, Ithaca NY). This bioacoustics program is used to perform conservation research and as such is adaptable to a wide range of animal AB. Nine APs were measured: six temporal and three spectral (Table 16). Feeding AB (lick, chew, drink) were produced by offering a compatible food item such as peanut butter, kibble, and chicken broth, respectively. Respiratory AB (cough and throat-clear (TC)) were produced by light digital palpation of the trachea. Vocalization AB (growl, whine, bark) were produced via multiple means including command, having the owner exit the collection area, and/or withholding a toy or food item.

\section{Manual identification of acoustic behaviors}

A trained reviewer $(\mathrm{MG})$ blinded to the data sets evaluated randomized waveform and spectral images. Randomized digital images of waveform and spectral data were presented to the reviewer. The reviewer then saved each image under its assigned AB. Each image was classified as one of eight possible AB (cough, chew, lick, bark, whine, drink, growl, or TC). This process was repeated to calculate intra-reviewer reliability. Correct group assignment (i.e., feeding, vocalization, or respiratory AB) was also 
assessed as for each AB.

\section{$\underline{\text { Statistical methods }}$}

Statistical analysis was performed using Past (version 3.21), SigmaPlot (version 14.0) data analysis software, and MedCalc (version 19). Descriptive statistics were performed where appropriate. A one-way Analysis of Similarity (ANOSIM) using a BrayCurtis similarity index was used to test for similarity between AB with a $p<0.001$ significance level. Where $\mathrm{AB}$ were found to be dissimilar, within group and between group evaluations were performed by a one-way ANOVA on Ranks. $\mathrm{P}<0.05$ was considered significant. A Wilcoxon rank-sum test was performed to assess for differences in $\mathrm{AP}$ between $\mathrm{AB}$ induced by tracheal palpation and subsequent coughs when more than one cough was produced after a single tracheal palpation. Normality was evaluated by Shapiro-Wilk test. Post-hoc analysis (Dunn's method or Bonferroni correction for multiple comparisons) was applied where appropriate. Kappa coefficients were calculated to assess intra-reviewer agreement for manual assignment of AB. Accuracy was recorded as percent of waveforms correctly identified.

\section{Results}

\section{$\underline{\text { Animals }}$}

Ten healthy employee-owned dogs were enrolled in this study with informed consent. Ages ranged from 2 to 12 years with a median (IQR) age of 5 years (3-7.5 years). Body weight $(\mathrm{kg})$ ranged from 4.2 to $28 \mathrm{~kg}$ with a median (IQR) body weight of $8.25 \mathrm{~kg}(5.15-20.4 \mathrm{~kg})$. Body condition score (9 point scale) ranged from 4 to 7 with a median (IQR) BCS of 5 (5-5). Five dogs were spayed females, four were castrated males, and one was an intact female. Breeds represented included: Mixed breeds $(n=2)$, 
Miniature Dachshunds (n=2), Beagle $(n=1)$, Miniature Pinscher $(n=1)$, Husky $(n=1)$, Catahoula Leopard Dog $(n=1)$, Yorkie $(n=1)$, Doberman Pincher $(n=1)$.

\section{$\underline{\text { Acoustic behaviors }}$}

Data were recorded from $\geq 3$ dogs per $\mathrm{AB}$. Respiratory AB, cough and TC, were recorded for 6 and 3 dogs respectively. The total number of waveforms/spectra evaluated for each $A B$ are as follows: cough $(n=24)$, chew $(n=104)$, lick $(n=73)$, bark $(n=43)$, whine $(n=35)$, drink $(n=89)$, growl $(n=17), T C(n=8)$. Representative examples for each AB are presented in Figure 19. Acoustic parameter data pertaining to each AB are found in Table 16. An analysis of similarity found cough to be dissimilar $(\mathrm{p}<0.0001)$ to all other AB with the exception of throat clear $(\mathrm{p}=0.09)$. The vocalization $\mathrm{AB}$, growl and whine, were acoustically similar, as were TC and drink, with p values of 0.04 and 0.06 respectively. All other AB were significantly acoustically dissimilar (Table 17). No between-subject differences were identified between cough and TC groups for any parameter. All other behaviors showed statistically significant within-group variation $(P<0.001)$. Cough and TC were found to be distinguishable from every other evaluated $\mathrm{AB}$, with significant differences in two or more of the analyzed parameters $(P<0.001$ for each; Table 18). No differences were detected between $\mathrm{AB}$ induced by tracheal palpation and subsequent respiratory $\mathrm{AB}$ for any $\mathrm{AP}$. No significant differences for cough or TC were found for any AP based on patient demographics. Randomized AB were correctly assigned by a blinded reviewer with $96.5 \%$ accuracy. This arm of the experiment was performed twice with kappa coefficient calculations being performed for each acoustic behavior and again for group assignment: feeding, vocalization, and respiratory behaviors. The kappa coefficient for individual behaviors was 0.76 suggesting moderate agreement. For group assignment 
the kappa coefficient was 0.97 suggesting near perfect agreement. Respiratory behaviors were assigned to the correct group $100 \%$ of the time with a kappa coefficient of 1.0 . (Table 19)

\section{Discussion}

In this study, we found that mesocephalic dogs generated consistent respiratory $\mathrm{AB}$ for cough and $\mathrm{TC}$, which could be differentiated from non-respiratory $\mathrm{AB}$ using temporal and spectral descriptors. Cough and TC are important respiratory protective mechanisms acting both as symptoms of disease and important markers of disease control. The practical use of cough as a marker of disease control is substantially hampered by subjectivity and placebo effect. Non-invasive acoustic monitoring would be valuable for future objective assessment of disease control in dogs with chronic respiratory disease while opening the door for objectively evaluated placebo-controlled clinical trials.

For many respiratory conditions, cough is the primary marker of disease severity and is used by clients and clinicians alike to judge response to therapeutic intervention $(285,286)$. Further, in conditions where structural airway disease represents the primary etiology (e.g., collapsing trachea), pharmacologic cough suppression is the cornerstone of medical intervention $(292,293)$. Though the use of antitussives is widespread, there is little objective data to support clinical efficacy, instead relying on perceived clinical improvement. Unfortunately, these methods of assessment are highly subjective. In people, the placebo effect accounts for up to $85 \%$ of the efficacy of some over-thecounter cough medications (294). Studies in people evaluating accuracy of medical selfreporting have found $23-40 \%$ respondents reported fabricated results both for objective disease metrics and maintaining interventions initiated in hospital $(287,295)$. In 
veterinary medicine, it is possible to introduce an even greater degree of subjectivity and bias as patient reporting becomes the responsibility of the client. This is supported by studies in human pediatric patients, where patient self-reporting is not possible. In these studies the accuracy of cough reporting by care givers was poor compared to objective cough monitoring $(296,297)$. These confounding variables have the potential to negatively impact the ability of the clinician and/or researcher to judge disease severity and accurately assess response to therapy. This highlights the need for objective respiratory biometrics for assessment of clinical patients and for placebo-controlled clinical trials.

In people, assessment of cough acoustic recordings provides an accurate means to assess cough frequency, in part because the cough $\mathrm{AB}$ has been well characterized (134, 291, 298, 299). The same has not been previously performed for dogs. In this study, we successfully collected respiratory, feeding, and vocalization AB. Dogs were minimally restrained during data collection in a quiet room to reduce ambient noise interference as ambient noise has been a challenge for $\mathrm{AB}$ characterization in people (300). Dogs were otherwise encouraged to freely engage in each $\mathrm{AB}$ apart from cough and $\mathrm{TC}$, which were manually induced. Free-feeding and minimal handling was considered important to ensure that the $\mathrm{ABs}$ recorded as a part of this study are representative and not unduly influenced by restraint or force feeding. For respiratory behaviors, when a single palpation event resulted in a series of respiratory $\mathrm{AB}$, the initiating respiratory $\mathrm{AB}$ (induced by tracheal palpation) and each subsequent respiratory $\mathrm{AB}$ in the series were evaluated for significant differences in AP. This comparison demonstrated that coughs induced by tracheal palpation and subsequent respiratory $\mathrm{AB}$ were acoustically 
indistinguishable in healthy dogs. This mirrors studies in people evaluating spontaneous and induced cough and suggests that coughs induced by tracheal palpation are suitable for objective evaluation $(301,302)$. As such, induced respiratory $\mathrm{AB}$ were grouped with their respective spontaneous $\mathrm{AB}$ for further analysis. Though this finding should be reevaluated in dogs with respiratory disease, it suggests in-hospital evaluation of cough sensitivity may be performed without significantly altering the acoustic profile of respiratory $\mathrm{AB}$ in dogs.

This study effectively demonstrates that healthy mesocephalic dogs generate respiratory $\mathrm{AB}$ that are consistent across dogs. Specifically, in this pilot study respiratory $\mathrm{AB}$ are not affected by demographic data such as BCS, weight, and breed, and are distinguishable from other $\mathrm{AB}$ based on objective AP. Though larger validation studies are needed to confirm these finding, this consistency suggests potential utility for respiratory sound analysis as an objective respiratory biometric. Similar to studies in people, TC was found to be qualitatively similar to cough and could not be distinguished based on the AP measured (303). Though distinct physiologically, both cough and TC are considered respiratory protective behaviors. TC is likely to be considered a "small cough" by most lay-evaluators (clients) and still has the potential to suggest the presence of diseases such as extra-esophageal reflux or post-nasal drip, which cause cough through stimulation of the larynx (304). For this reason, discriminating cough from TC was not considered necessary for clinical utility and was therefore not a significant limitation in this study.

Cough sound processing in people is either performed manually or through automatic sound processing algorithms. Automatic processing algorithms are often 
challenged by the need to distinguish between respiratory sounds and ambient noise and talking (305). As such, manual evaluation is still an integral part of acoustic analysis in humans. This study demonstrated that $\mathrm{AB}$ in dogs could be identified with $96.5 \%$ accuracy by a trained reviewer blinded to the data sets. If $\mathrm{AB}$ were grouped by function (respiratory, vocalization, or feeding behaviors), respiratory $\mathrm{AB}$ were correctly identified $100 \%$ of the time. Collectively these data suggest that manual identification of $\mathrm{AB}$ in dogs is feasible.

Limitations of this study included a relatively small sample size. Multiple AB were collected for each dog to minimize type 2 error. Also, the current collection protocol is limited to the hospital setting with simultaneous induction of the $\mathrm{AB}$ and data recording over short periods of time. Ambulatory collection devices have been developed for people and are useful for longer term monitoring. Ambulatory collection in dogs, allowing for spontaneous $\mathrm{ABs}$ in natural settings, may prove to be easier than in humans as dogs do not generate the same range of vocalization behaviors. Acoustic behaviors including sniffing, sneezing, and reverse sneezing were not included in this pilot study. When recorded by a microphone positioned over the larynx, sniffing did not reliably result in discrete $\mathrm{ABs}$ distinguishable from background noise and were therefore excluded from objective evaluation in this pilot study. Sneezing and reverse sneezing could not be induced in the healthy dogs in this study but should be included in future studies validating acoustic cough recording for clinical use. $(139,288,306,307)$.

\section{Conclusions}

Objective metrics of cough, an important clinical manifestation of many respiratory disorders, are lacking in dogs. Cough frequency has the ability to significantly 
impact clinical decision making, result in significant patient morbidity, and impact a client's perception of quality of life for their animal. In this study, respiratory AB were demonstrated to be statistically distinct based on objective metrics and can be accurately identified by manual analysis. This study represents a proof of concept for the objective assessment of cough frequency by identifying respiratory $\mathrm{AB}$ in healthy dogs. 


\section{CHAPTER 8}

\section{CONCLUSIONS AND FUTURE DIRETIONS}

The studies described herein highlight the complex inter-relationship between swallowing and respiration. The frequent identification of abnormal swallowing in the studied dogs supports a possible contribution from dysphagia, reflux, and aspiration in the pathogenesis of canine respiratory disease. This underscores the need for a multimodal diagnostic and therapeutic approach in dogs with aerodigestive disease as well as well designed, objectively evaluated, prospective studies in the veterinary literature. Historically this aim has been hampered by a lack of clinical recognition, diagnostic limitations, and a lack of objective monitoring strategies in clinically affected patients. This dissertation presents our responses to these limitations.

The lack of clinical recognition for aerodigestive disease and aspiration associated respiratory syndromes (AARS), a subclassification of aerodigestive disease, is multifactorial with a likely contribution from an overreliance on thoracic radiography and the fact that many affected patients present without clinical evidence of GI disease. $(7,8$, 308) Diagnostic evaluation of respiratory disease in dogs typically begins with thoracic radiogaphs. Though radiographs have the benefit of being widely available they lack sensitivity when investigating aerodigestive disease and provide almost no functional information. $(109,308,309)$ Videofluoroscopic swallow studies (VFSS) represent the criterion standard for diagnosis of dysphagia in dogs, however, like radiographs, these have significant limitations. $(239,310-312)$ The reliance on force-feeding in lateral recumbancy carries increased risk of aspiration pneumonia such that it is considered contraindicated in certain dysphagic disorders (e.g., megaesophagus). Recently, the 
validation of an upright free-feeding VFSS protocol has permitted investigation of a broader range of dysphagic disorders while limiting the risk of aspiration pneumonia and increasing physiologic relevance of the data obtained.(239)

This expanded use of VFSS in dogs with chronic cough (Chapter 2) has provided important information on the prevalence and breadth of aerodigestive disease in dogs. Our studies support aerodigestive disease as a common contributor to chronic cough in dogs with nearly $81 \%(25 / 31)$ of patients presenting solely for cough having evidence of swallow dysfunction detected by free-feeding VFSS. This included 11 dogs with normal thoracic radiographs. Of these 31 dogs, only 7 had evidence of aspiration pneumonia on thoracic radiographs. This study emphasizes a previously under-recognized and common canine population with numerous different alimentary tract diseases causing or contributing to cough. This demonstrates that, like people, aerodigestive disease in dogs is diverse and may present in the absence of gastrointestinal signs. These findings also suggest that a high index of clinical suspicion is required in addition to a multimodal approach for evaluation of respiratory patients due to thoracic radiography being insufficient to detect aerodigestive disease. In dogs with both respiratory and GI causes for cough (i.e., mixed disorders) each likely contributes to disease progression. As such, treatment in dogs with mixed respiratory and alimentary disease is likely to be multimodal reflecting the contribution of alimentary tract disease in such cases. Given the high prevalence of dogs with VFSS swallow abnormalities in our study, clinical suspicion for aerodigestive diseases should be high with consideration being also given to dogs with chronic cough and not just those with evidence of gross aspiration.

In addition to providing necessary information on the relationship between 
respiration and swallowing in coughing dogs, the broader clinical use of VFSS has resulted in improved characterization of a clinically important dysphagic disorder in dogs: megaesophagus (Chapter 3). Using this technique, our group successfully identified a subpopulation of dogs with a failure of the lower esophageal sphincter (LES) to relax in response to a pharyngeal swallow (i.e., LES achalasia-like syndrome). Similar functional obstructions of the LES have been identified in people (i.e., LES achalasia). Identifying this condition in dogs was considered critically important as functional LES obstructions in people represent one of the only forms of esophageal dysphagia with a demonstrated response to targeted intervention. Though the criterion standard for diagnosis in people is high-resolution manometry, its use in dogs is significantly limited.(18) Reliance on this technique would significantly limit clinical actionability in dogs, as few patients would be able to be evaluated. This made finding an alternative more widely available means to identify this population of dogs highly clinically relevant. Though interpretation will require high quality diagnostic studies and practice, with the guidelines developed herein, a diagnosis of LES-AS may be possible for a far greater number of dogs. This is especially important because our group later demonstrated that dogs with LES-AS, like people with LES-achalasia, respond to intervention directed at relieving the obstruction at the LES (Chapter 4).(313)

In our study mechanical dilation and botulinum toxin A (BTA) injections at the LES resulted in $100 \%$ perceived clinical improvement by owners as well as significantly increased body weight, body condition score, and significantly reduced regurgitant frequency.(313) These subjective and objective clinical assessments were supported by improved swallowing parameters as detected by VFSS. In these cases, improved gastric 
filling and esophageal clearance were identified despite minimal improvement in esophageal motility further implicating the LES in the pathogenesis of disease for the subpopulation of dogs. Though effective, the results of this intervention were temporary which limits the use of mechanical dilation and BTA injections to the role of adjunctive diagnostic or as a stopgap measure in patients awaiting definitive treatment. A definitive approach was undertaken in a subpopulation of dogs who responded favorably to mechanical dilation and BTA injections at the LES. A Heller myotomy with Dor fundoplication was found to be similarly effective and may represent a long-term intervention in dogs with LES-AS.(313) The findings of this study provide hope for a population of dogs with a disease for which there have been historically very few therapeutic options.

Though VFSS are beneficial for the detection of dysphagia and macroscopic aspiration events, they lack the sensitivity to detect small volume aspiration (i.e. microaspiration) events. Microaspiration is a significant contributor to the pathogenesis and progression of chronic respiratory disease in people and is frequently linked to reflux disease. $(82,234)$ Reflux scintigraphy has been used historically to reflux and cumulative aspiration in people and in our study was successfully adapted for use in dogs. $(127,130)$ Using reflux scintigraphy (Chapter 5), we identified that extra-esophageal reflux (EER) and pan-esophageal reflux, gastroesophageal reflux (GER) extending the length of the esophagus, occurred frequently in healthy asymptomatic dogs. These findings mirror results in people where healthy, asymptomatic people frequently have reflux events reaching the proximal esophagus with a smaller number of reflux events extending beyond the esophagus as EER. Interestingly and counter to the literature in people, 
aspiration was not detected in any healthy dog. In this study, we successfully adapted a technique capable of detecting small volume and cumulative reflux events for use in dogs and provided normative data necessary for interpreting findings in patients with aerodigestive or AARS. Prospective studies in dogs with chronic respiratory disease would substantially contribute to our understanding of disease pathogenesis in dogs with aerodigestive disease.

Other techniques used to detect extra-esophageal reflux and aspiration involve reflux biomarkers where digestive proteins are detected in sputum, oropharyngeal swabs, or bronchoalveolar lavage fluid (Chapter 6).(100, 122, 133, 280) This study utilized liquid chromatography mass spectrometry (LC-MS) to characterize the canine gastric fluid proteome and compare this to the oropharyngeal proteome in healthy dogs, vomiting/regurgitating dogs, and coughing dogs. This study found that pepsin A, a frequently used biomarker of reflux and aspiration in people, was a poorly abundant protein in canine gastric fluid. Instead, canine gastric fluid was dominated in abundance by pancreatic proteins. This finding implies that gastroduodenal reflux is a frequent occurrence in dogs and that these pancreatic proteins may play a role in AARS and should be considered as potential biomarkers of reflux and aspiration. Importantly variability in protein abundance between dogs likely limits the utility of individual biomarkers though evaluation of biomarker panels is warranted. Of additional interest was that the oropharyngeal proteome differed in health and disease both in terms of individual protein composition as well as biological function. These combined findings suggests clinical utility for protein biomarker panels for detection of reflux and aspiration in dogs encouraging future proteomic validation studies. 
In addition to limitations in detection of patients with aerodigestive disease, assessing disease severity and monitoring response to therapy is a significant challenge. Historical methods for judging the frequency and severity have lacked sensitivity and carry significant risk of bias.(297) This study proposed an objective method of cough monitoring by evaluating cough acoustic waveforms and spectrograms (Chapter 7).(301, 303) This study demonstrated that cough and throat-clear, another airway protective behavior, generated reliable and consistent acoustic behaviors (AB) which were distinguishable from other common $\mathrm{AB}$ (e.g., lick, chew, drink, growl, bark, and whine) by objective parameters. Individual $\mathrm{AB}$ classification was able to be accurately and reliably performed by a trained investigator. Objective monitoring of cough frequency has the potential to significantly impact clinical decision making allowing for more effective treatment and monitoring in patients with respiratory disease. This pilot study serves as a proof of concept for this novel monitoring strategy. Further studies evaluating automatically evaluated ambulatory devices are needed to increased utility in clinical patients.

Future research investigation in aerodigestive diseases should involve prospective evaluation of clinically affected dogs or dogs belonging to specific at-risk populations. Animals with known aerodigestive disease are important for broader validation studies for biomarkers of EER and aspiration as well as for determining distinguishing criteria between health and disease using VFSS and reflux scintigraphy. At risk populations include brachycephalic dogs which are considered predisposed toward several aerodigestive diseases including reflux and aspiration pneumonia. Specific evaluation of these dogs by VFSS and reflux scintigraphy may provide additional insight into 
aerodigestive disease through the use of brachycephalic dogs as an animal model of human disease.

Aerodigestive and AARS are the result of discordant respiration and swallowing. Despite a high prevalence for such disorders in dogs presenting for respiratory signs these cases are likely under-recognized in dogs presenting for cough in clinical veterinary practice. Advanced diagnostic and monitoring tools including VFSS, scintigraphy, biomarker analysis, and objective respiratory biometrics are needed to improve recognition and treatment of this population. Further, as both dogs and people have evidence aerodigestive disease, using a One Health approach will increase the opportunity for bidirectional advancement in research. 


\section{Appendix}

\section{Figures}

Figure 1

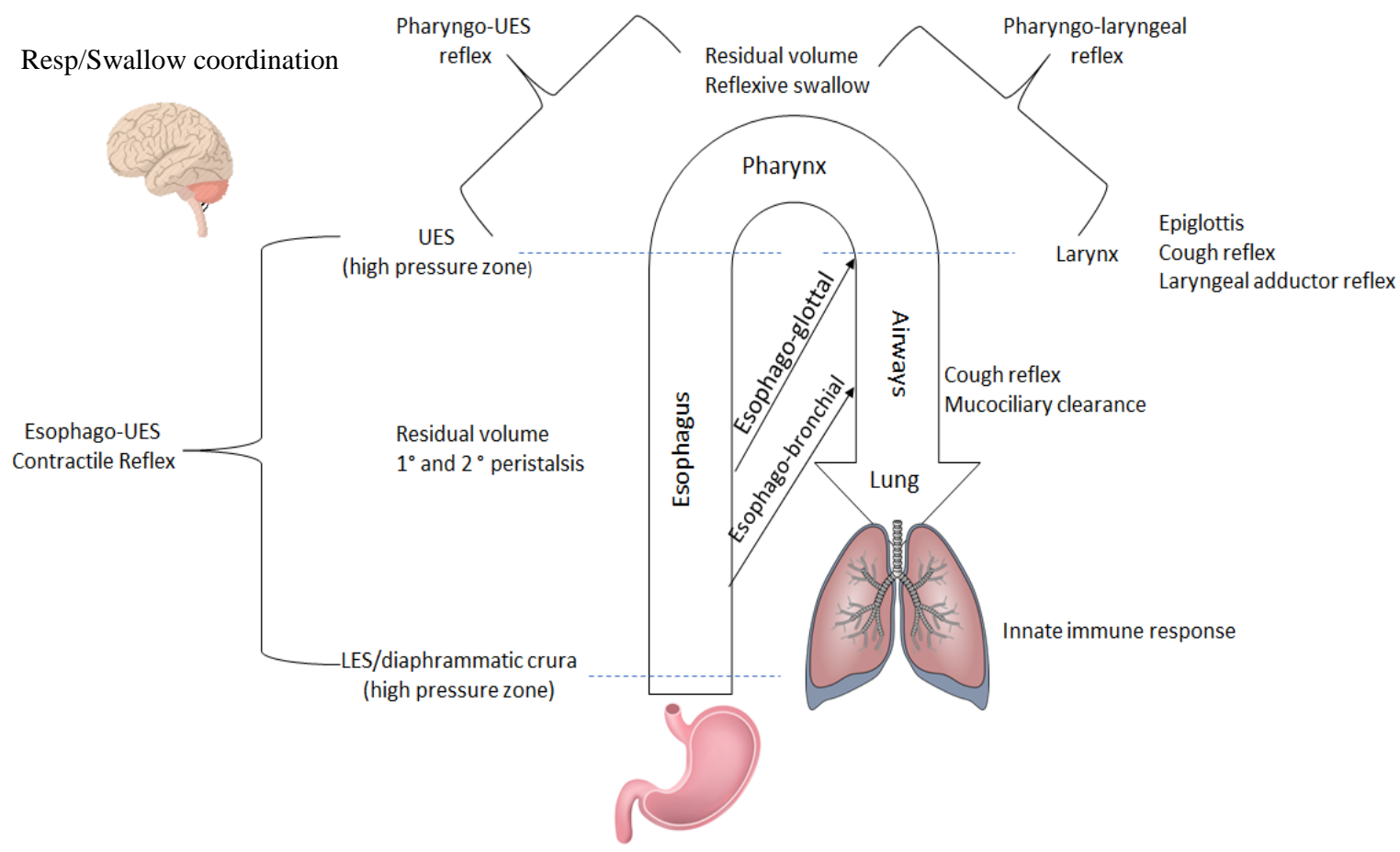

Figure 1: This figure summarizes airway protective mechanisms against aspiration. 
Figure 2

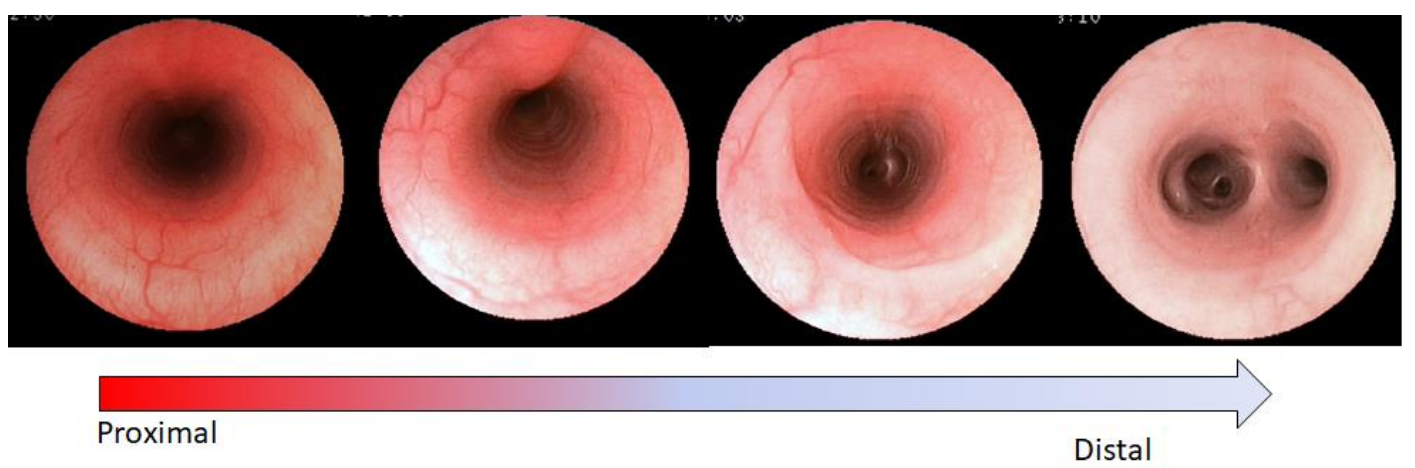

Figure 2. The tracheal stripe is an endoscopic finding characterized by proximal erythema that dissipates distally toward the carina. Changes were noted prior to intubation. Oxygen was administered via red rubber catheter alongside the bronchoscope. 
Figure 3

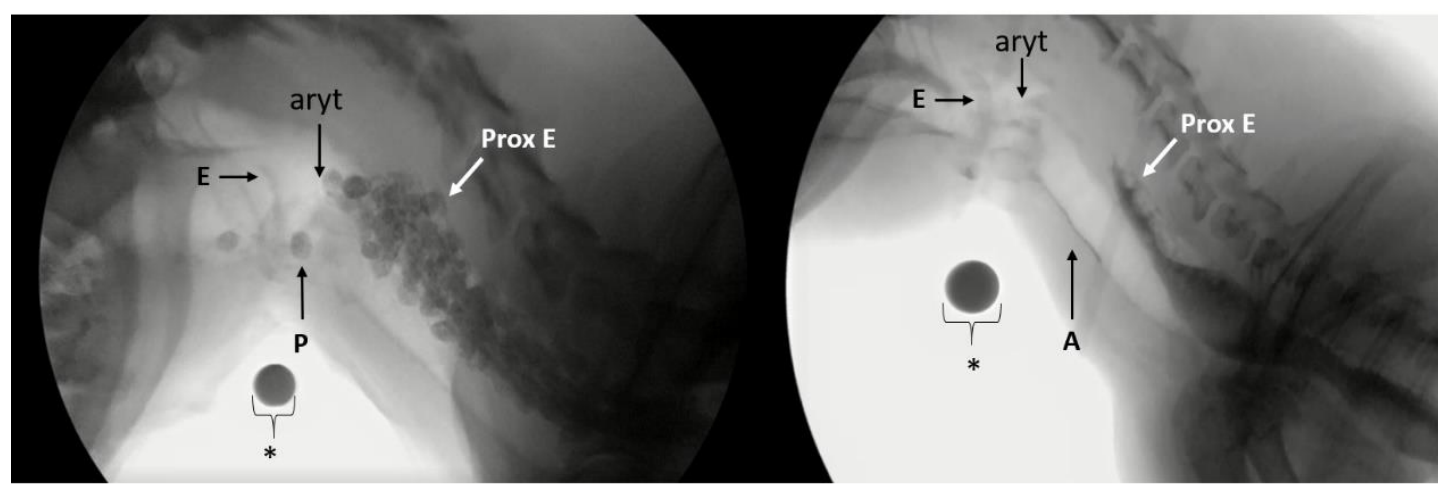

Figure 3: Videofluoroscopic swallow study example of penetration (P) and aspiration (A). The head is oriented to the left and the tail to the right. A $1 \mathrm{~cm}$ size marker is denoted by *. A food bolus is present in the proximal esophagus (Prox E). In the left image, a piece of barium-extruded kibble is found between the epiglottis (E) and the arytenoids (aryt) but does not extend past the larynx. In the right image, a line of aspirated contrast (A) is present in the ventral trachea after drinking liquid containing iohexol. A scoring system for penetration-aspiration is displayed in Table 3 . 
Figure 4

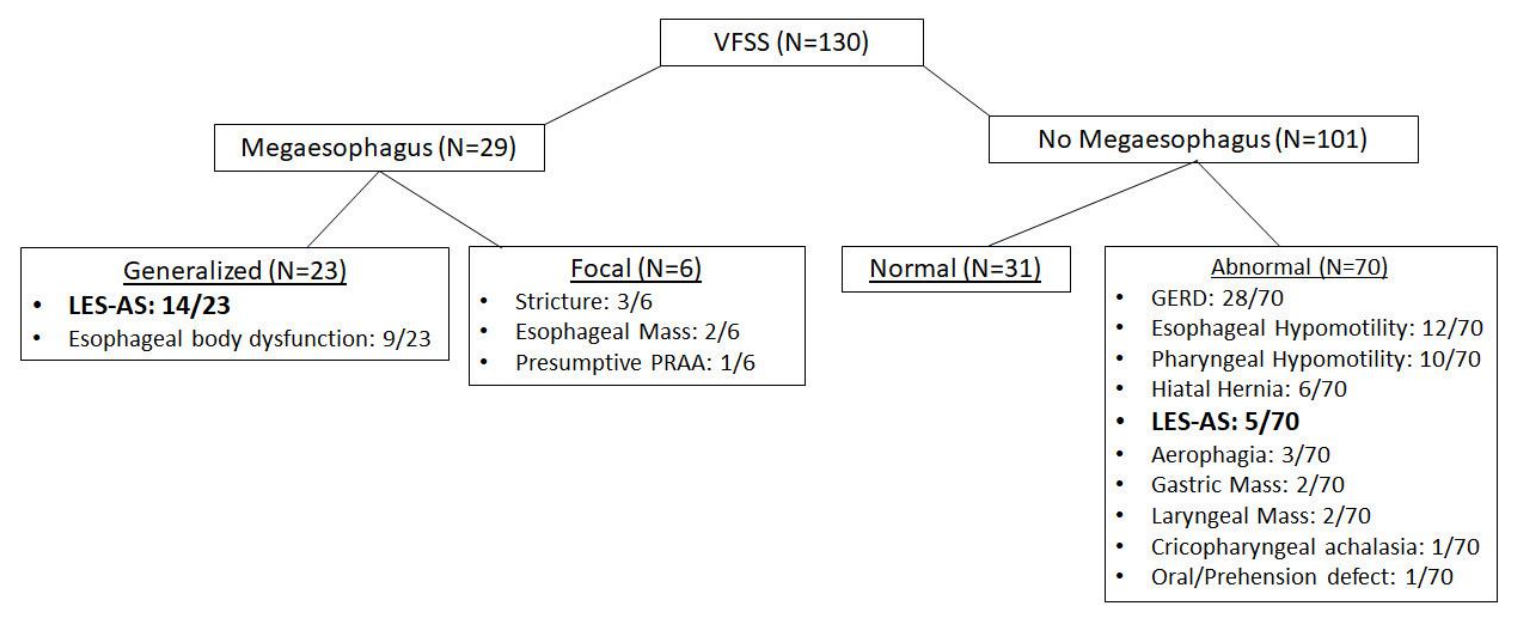

Figure 4: Final VFSS diagnosis for all patients evaluated by VFSS at the University MUVHC between April 2015-December 2017. 
Figure 5

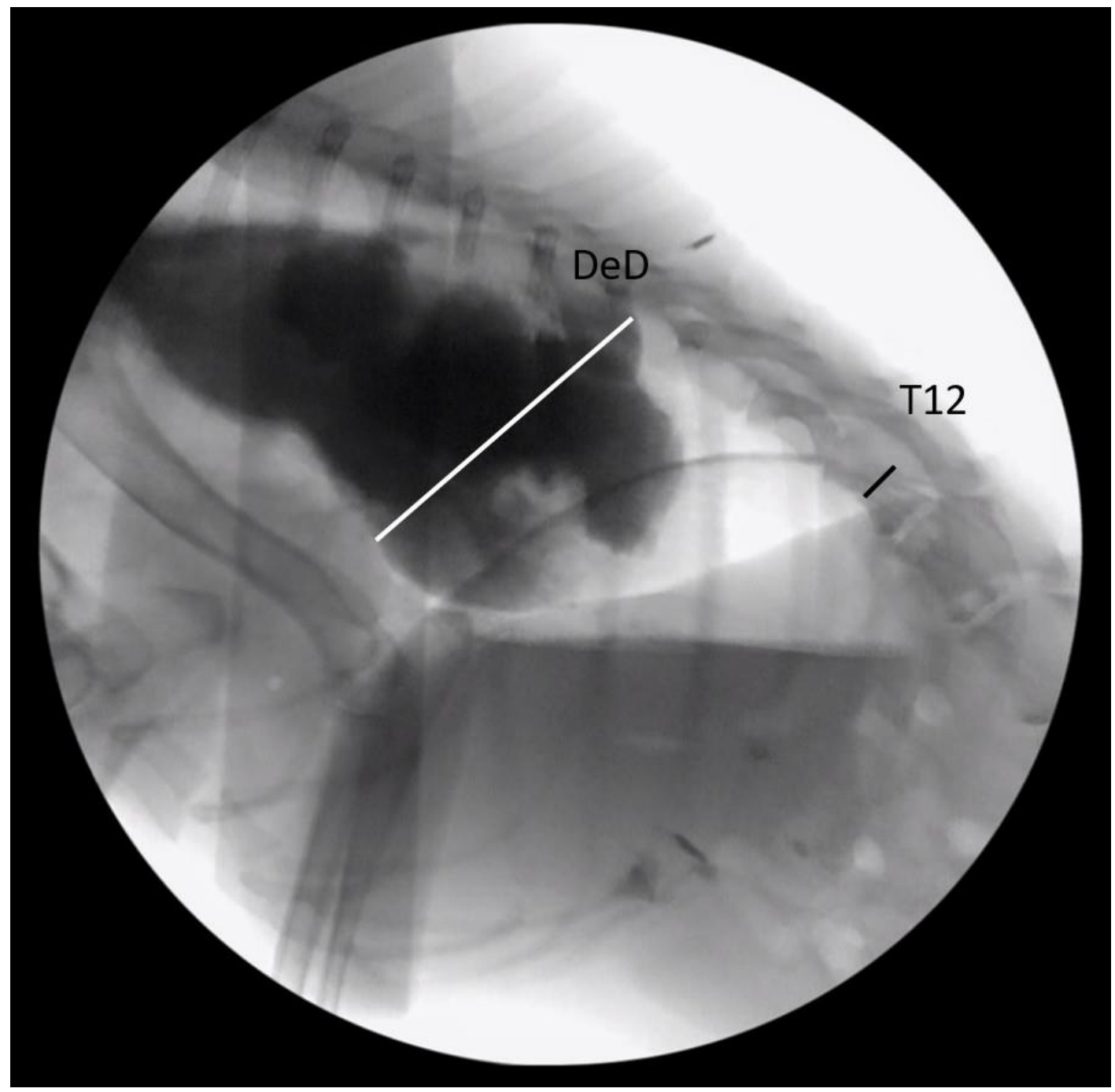

Figure 5: Maximal Distal esophageal diameter $(\mathrm{DeD})$ and the height of the T12 vertebral body are used to generate at DeD:T12 ratio. A comparison between this ratio (with $>4.7 \mathrm{x}$ being 94\% sensitive and 100\% specific for ME) and subjective assessment of ME showed perfect correlation with a kappa coefficient of 1 . 
Figure 6

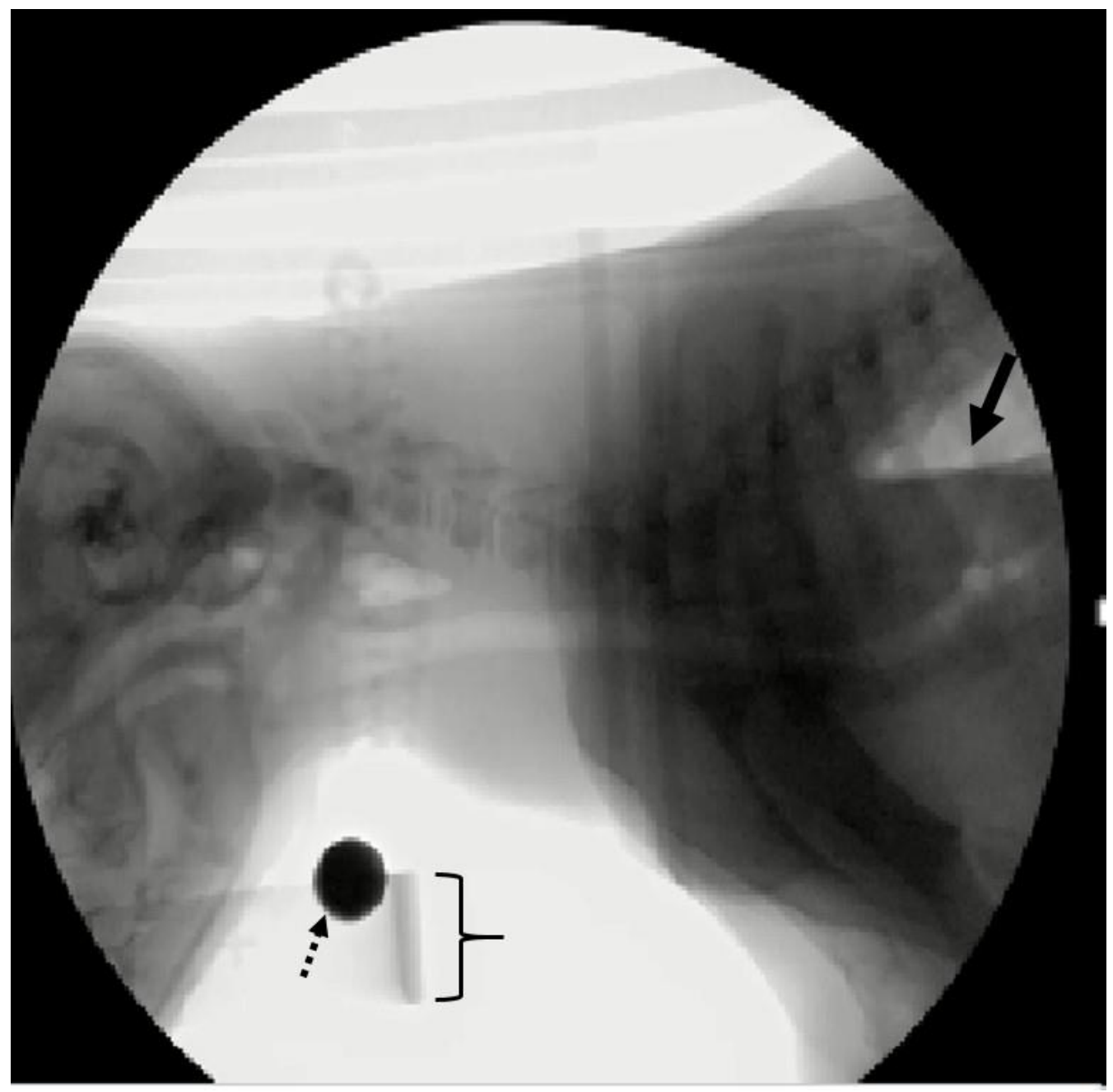

Figure 6: Cranial (Left), Caudal (Right). Lateral projection of a still image from a VFSS. A baseline fluid line (arrow) is visible in the esophagus after $\mathrm{a} \geq 12$ hour fast prior to administration of oral contrast material. The top lip of the food bowl is marked by brackets. The dashed arrow points to a $1 \mathrm{~cm}$ calibration marker worn around the patient's neck. 
Figure 7

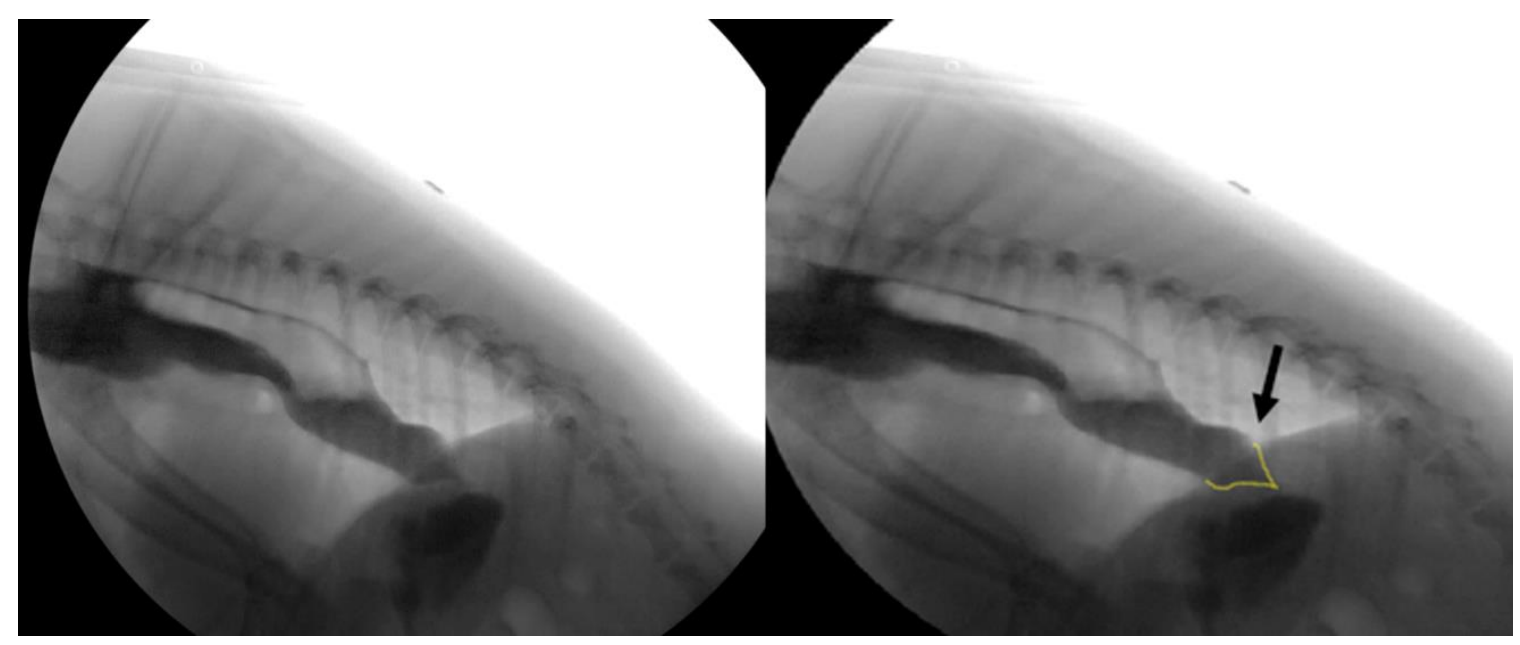

Figure 7: Cranial (Left), Caudal (Right). Fluoroscopic static image of the distal esophagus in a dog actively swallowing a pureed food consistency. This image demonstrates a narrowed contrast column resulting in an elongated taper through the LES (arrow). This appearance bears resemblance to a "bird beak" which is where this clinical feature received its name. 
Figure 8

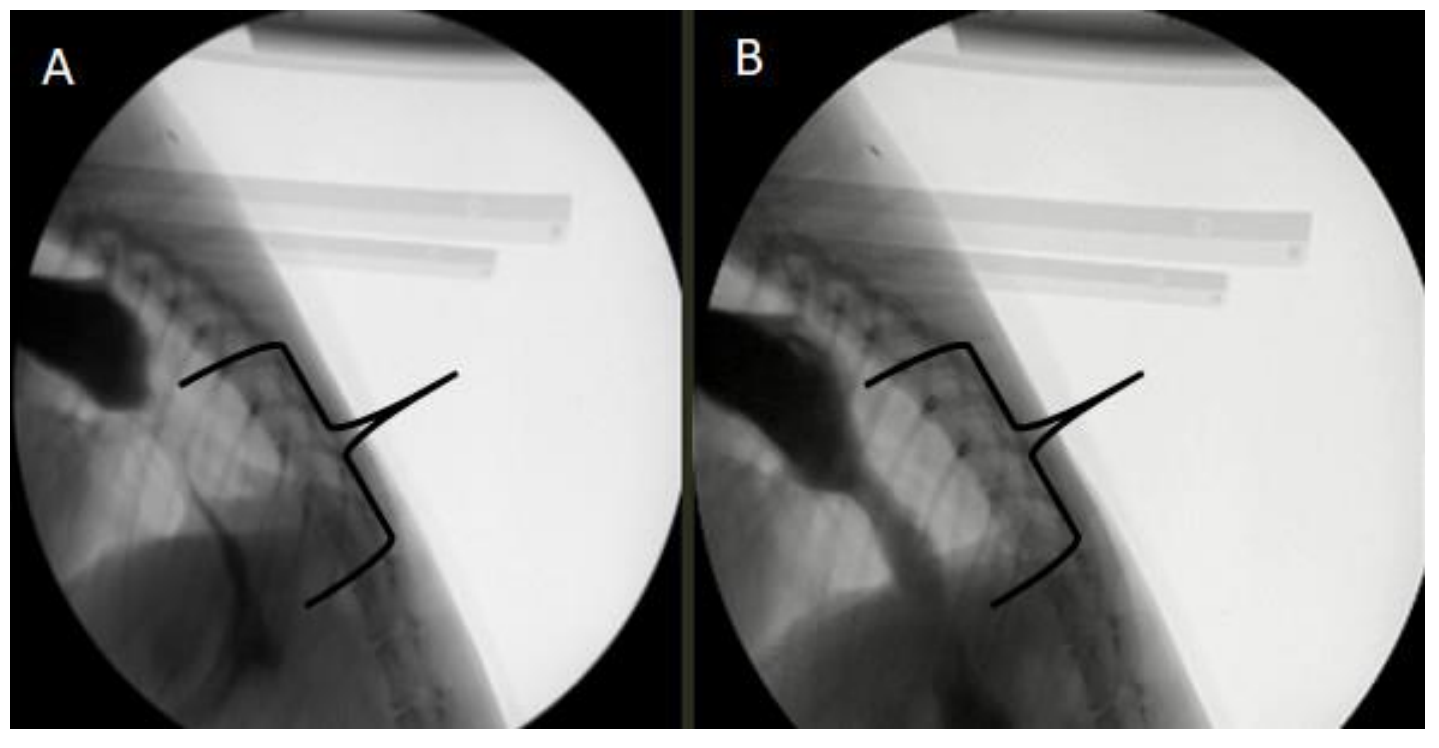

Figure 8: Cranial (Left), Caudal (Right). (A) A narrowed distal esophagus is present (region displayed by brackets) that (B) partially increases in diameter with increased hydrostatic pressure/gravity as the dog is sitting down. This demonstrates that failure of the LES to relax is secondary to a functional obstruction and can be overcome with enough hydrostatic pressure. 
Figure 9

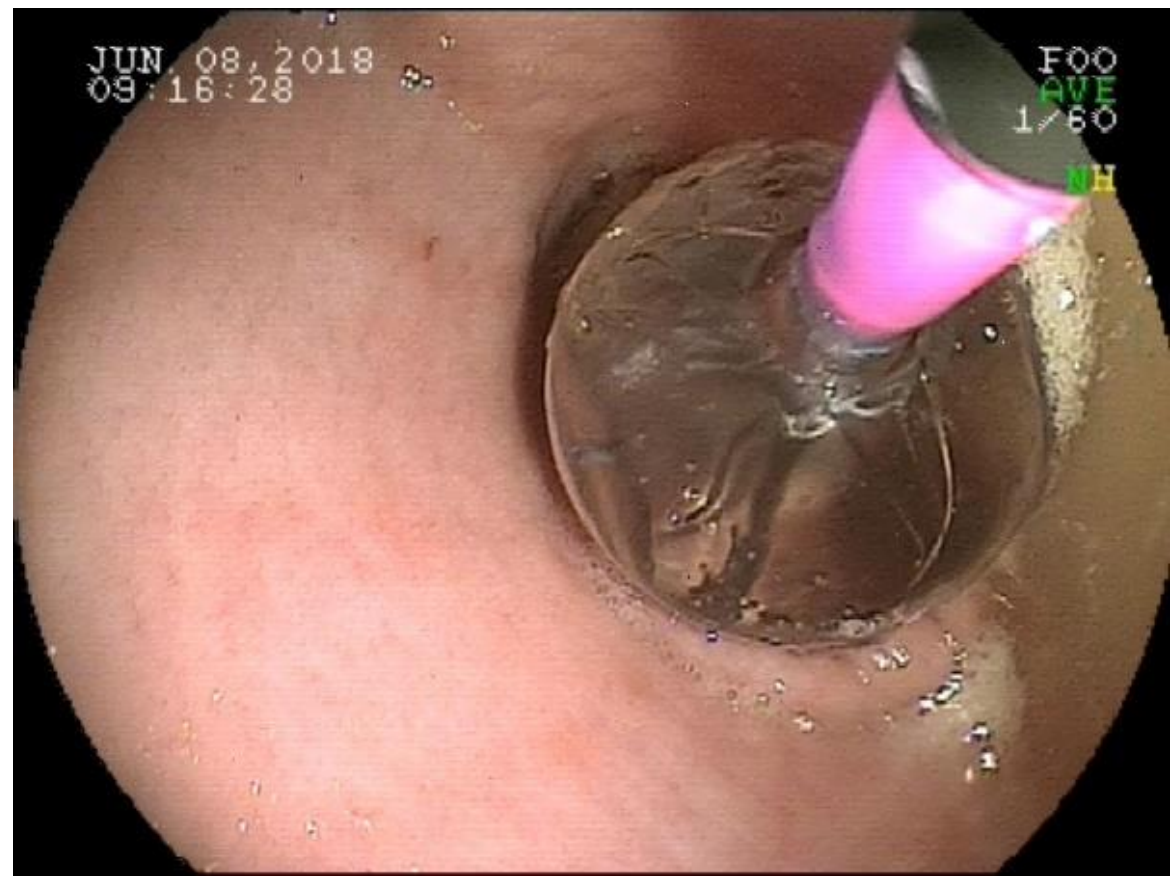

Figure 9: Balloon spanning the LES of a dog diagnosed with LES-AS. It is necessary for the balloon to span the entire LES for adequate dilation.(217) 
Figure $10 \mathrm{a}, \mathrm{b}$

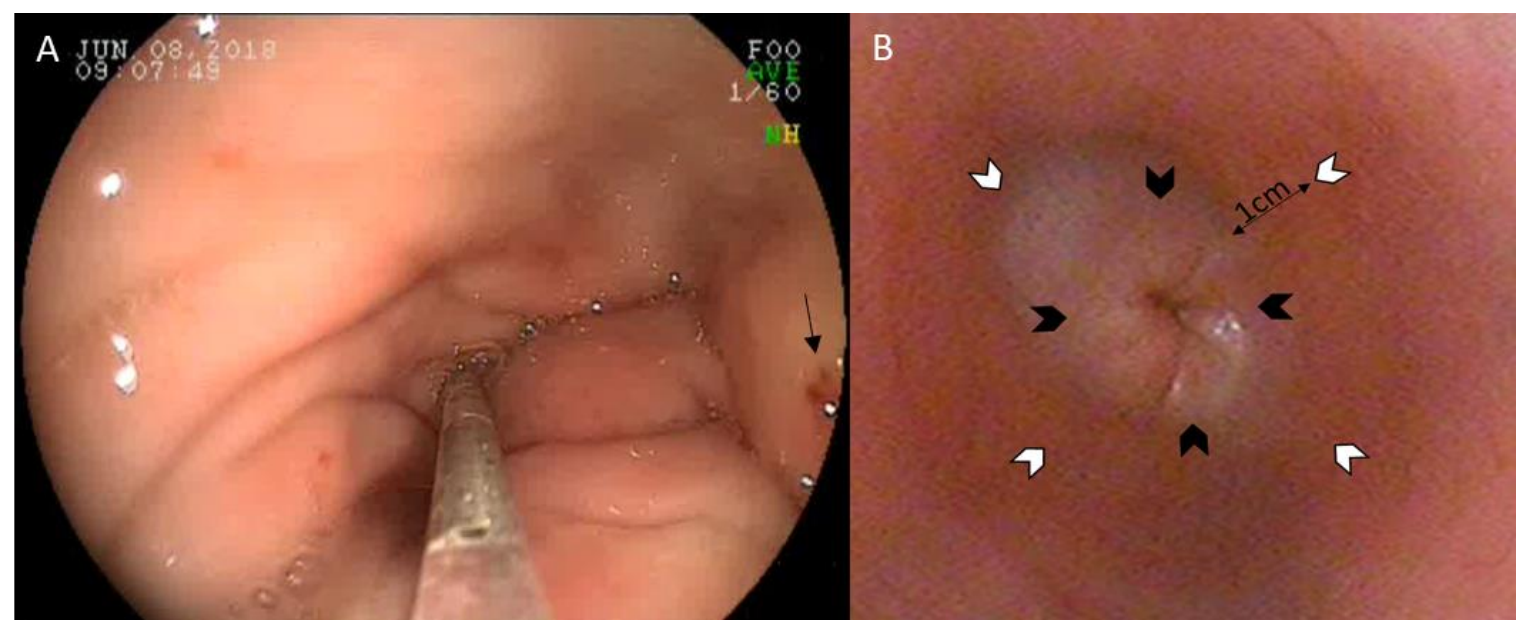

Figure 10 a,b: 2a) BTA $(40 \mathrm{U} / \mathrm{mL})$ is injected into 8 sites (4 U/site) circumferentially around the LES using an endoscopic injection needle. A small bleb should be visible after injection (black arrow). 2b) Four injections (Set 1, black arrowheads) are placed at $90^{\circ}$ immediately adjacent to the LES. The remaining 4 sites (Set 2, white arrowheads) should be placed at $90^{\circ} 1 \mathrm{~cm}$ distal to Set 1 . Set 2 should be rotated $45^{\circ}$ relative to Set 1 . 
Figure $11 \mathrm{a}, \mathrm{b}$

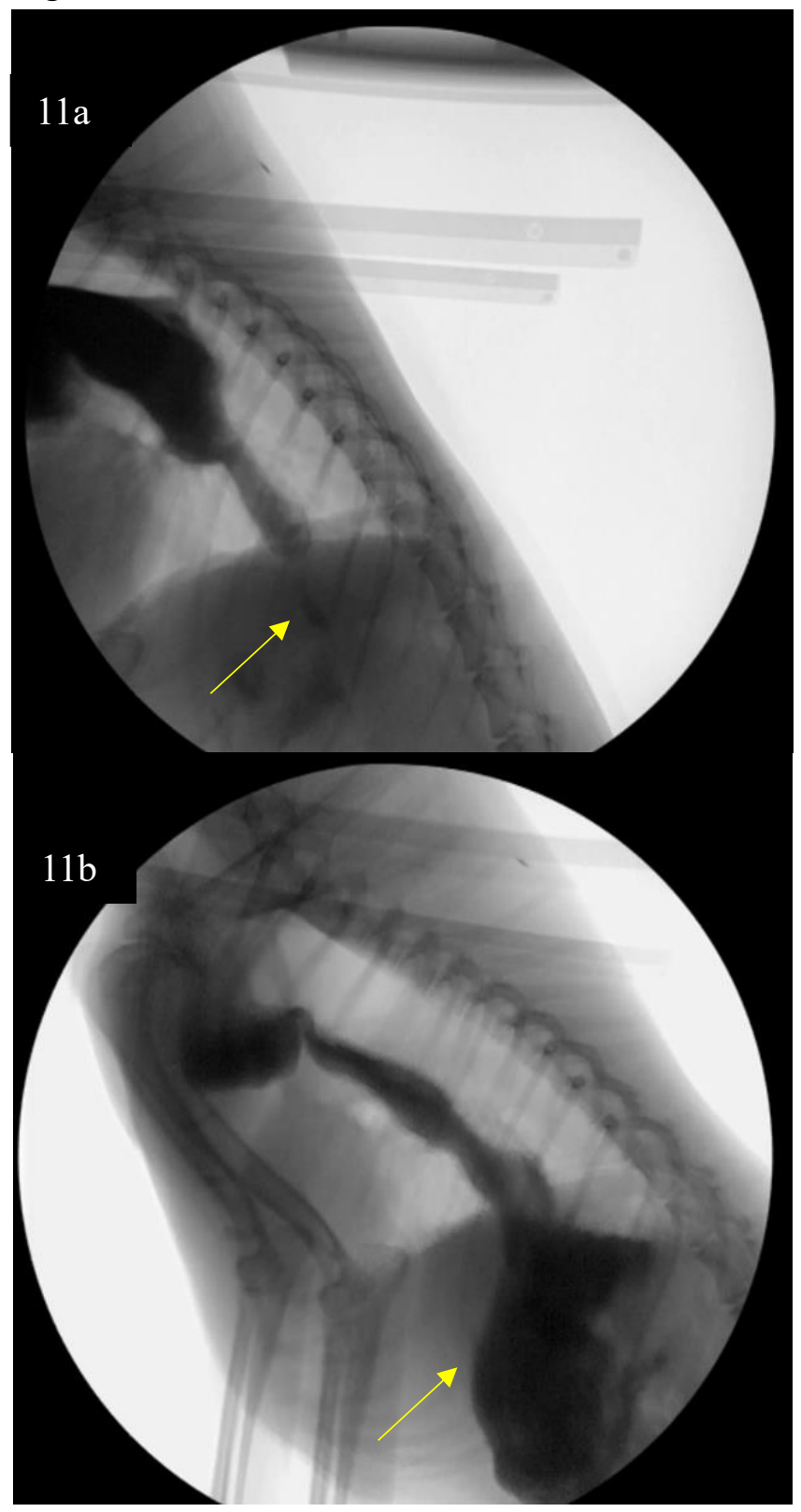

Figure 11 a,b: Still lateral image of a 6 year old female spayed mixed breed dog from a VFSS showing maximal gastric filling (arrows) in response to hydrostatic pressure before (11a) and after treatment with mechanical dilation + BTA. (11b). Only a small amount of gastric filling was appreciated prior to intervention; after treatment, there was a large amount of gastric filling observed, indicative of improvement in the functional LES obstruction. 
Figure 12 a-d

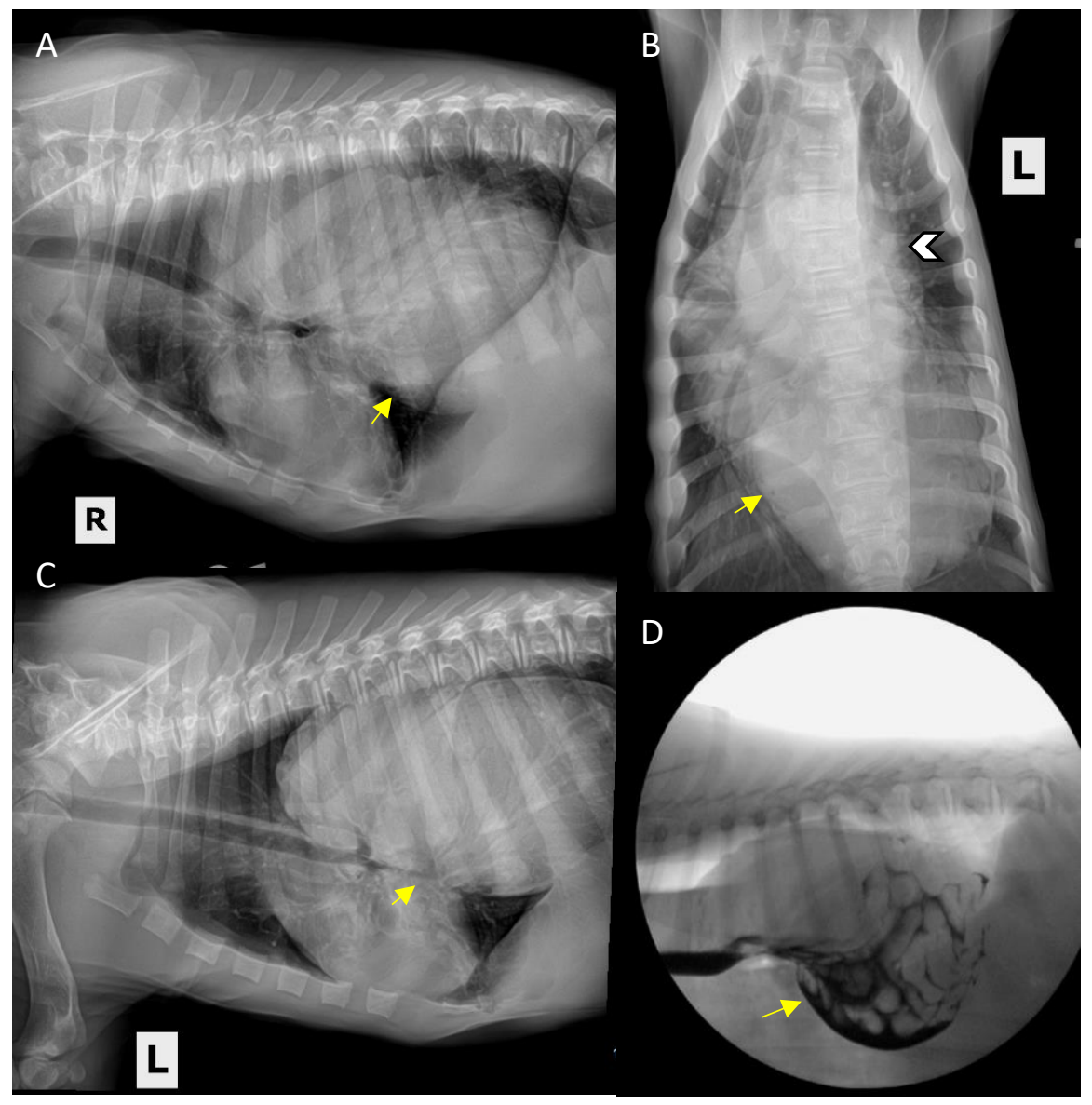

Figure 12 a-d) Three view (A: right lateral, B: dorsoventral, C: left lateral) thoracic radiographs of an 8 week old MI Irish Wolfhound presenting for lethargy, regurgitation and decreased appetite after treatment with mechanical dilation and BTA. A large soft tissue opaque structure is present in the distal esophagus (arrows). The cardiac silhouette is obscured by the esophageal contents (white arrowhead, 8b). 8d) A still lateral image from a VFSS showing a gastroduodenal-esophageal intussusception with Type IV hiatal hernia. The dog is in sternal recumbency and freely consumed liquid containing $25 \%$ Iohexol (350/mg iodine/mL). Contrast outlines intestinal loops within the dilated distal esophagus (arrow). 
Figure 13

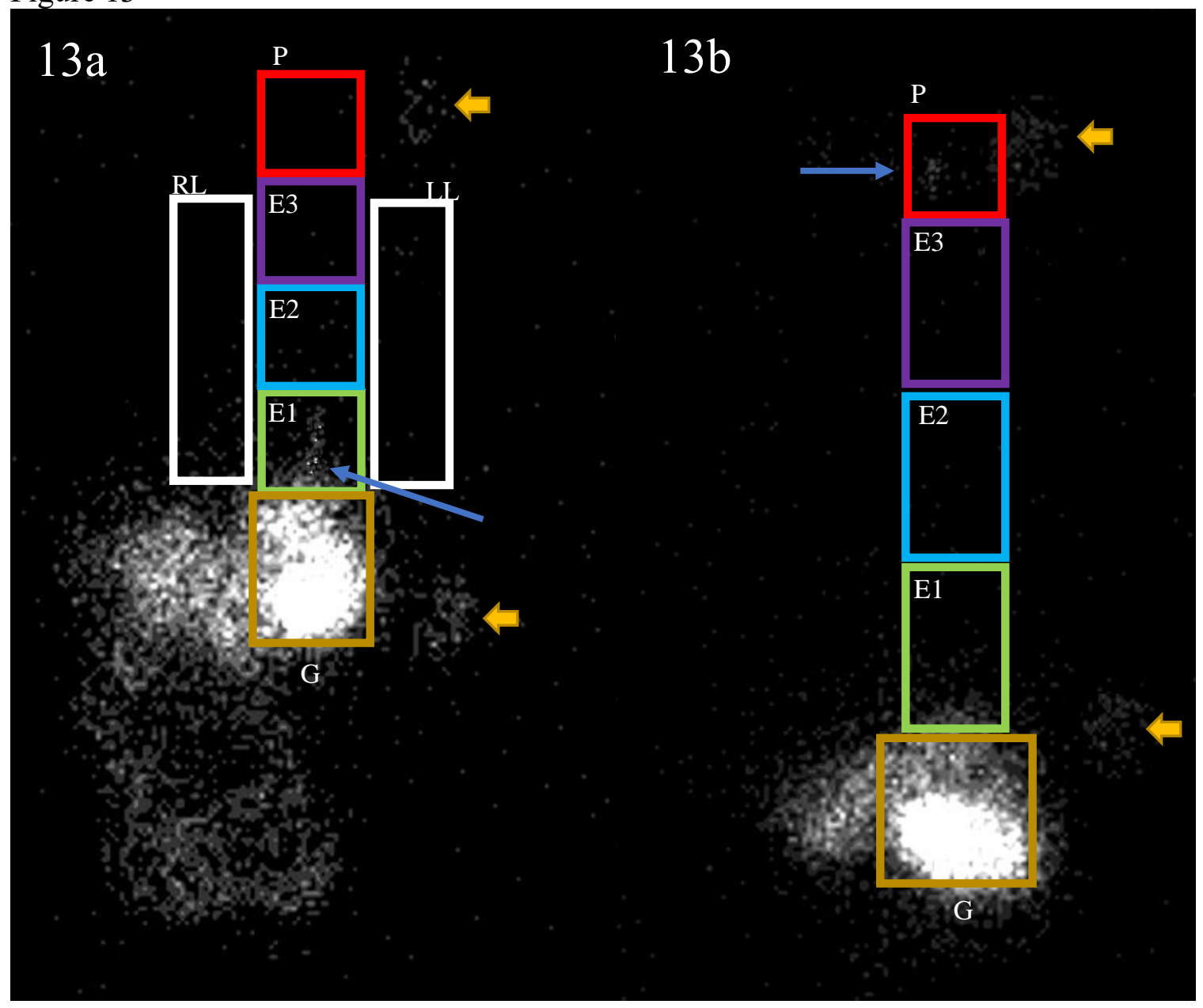

Figure 13a-b: Regions-of-interest (ROI) are drawn over dynamic images collected in dorsal (1a) and left-lateral (1b) recumbancy 30-min post-ingestion of a meal containing (3mCi) colloidal 99m-technetium phytate (99TcP). Lung ROI were not collected for dogs in lateral recumbancy due to summation of the right and left lung fields. Position markers at the level of the mandible and stomach are denoted by the arrowheads. Distal esophageal (E1) and pharyngeal (P) reflux are denoted by arrows in 1a and 1b respectively. Distal 3rd of the esophagus (E1), Middle $3^{\text {rd }}$ of the esophagus (E2), and proximal $3^{\text {rd }}$ of the esophagus (E3), pharynx (P), Left lung field (LL), right lung field $(\mathrm{RL})$, gastric $(\mathrm{G})$. 
Figure 14

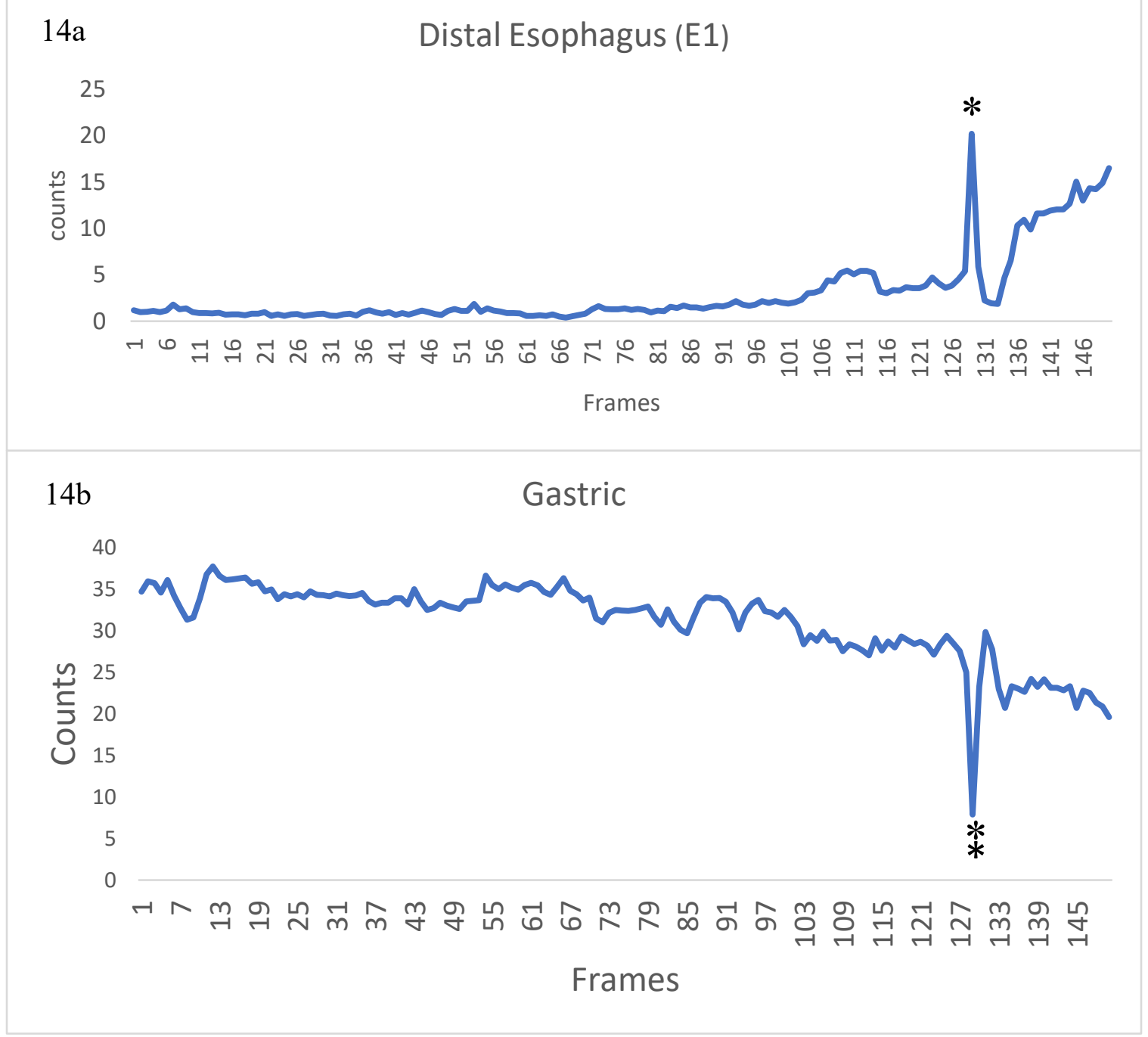

Figure 14a-b: Time-activity-curves are displayed for the distal esophagus (E1) (14a) and stomach (14b) respectively. 2a) A discrete reflux event representing counts $\geq 200 \%$ background is present between frames 128 and $131\left(^{*}\right)$. Data were collected at 2 seconds/frame. The duration of this reflux event is 3 seconds. This event corresponds to a decrease in gastric counts over the same time period $(* *)$ consistent with true gastroesophageal reflux. 
Figure 15

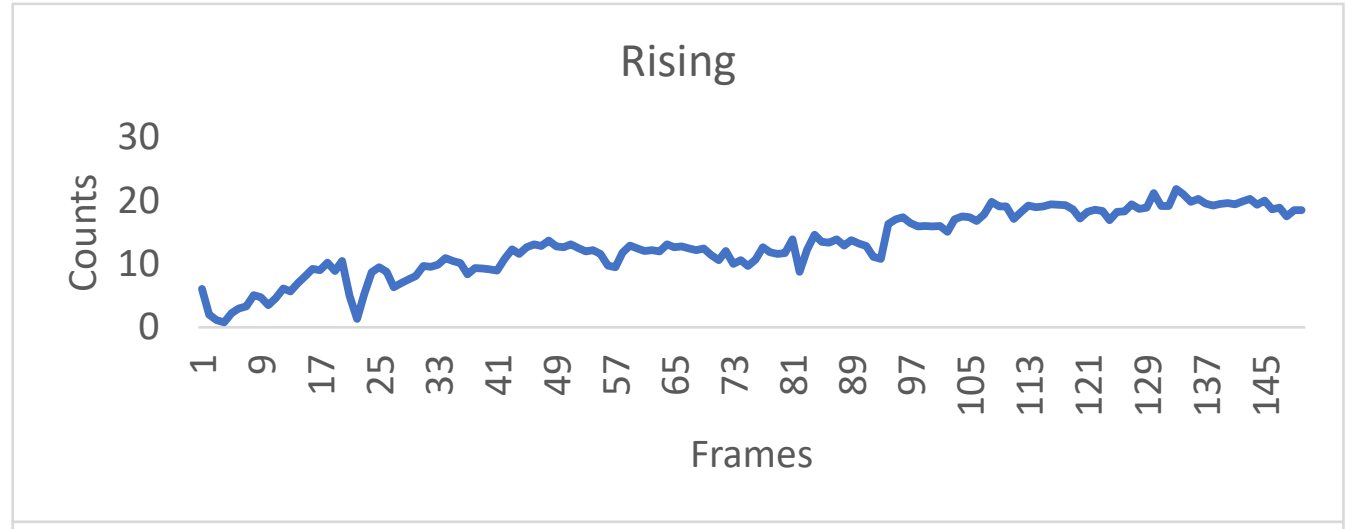

\section{Flat}

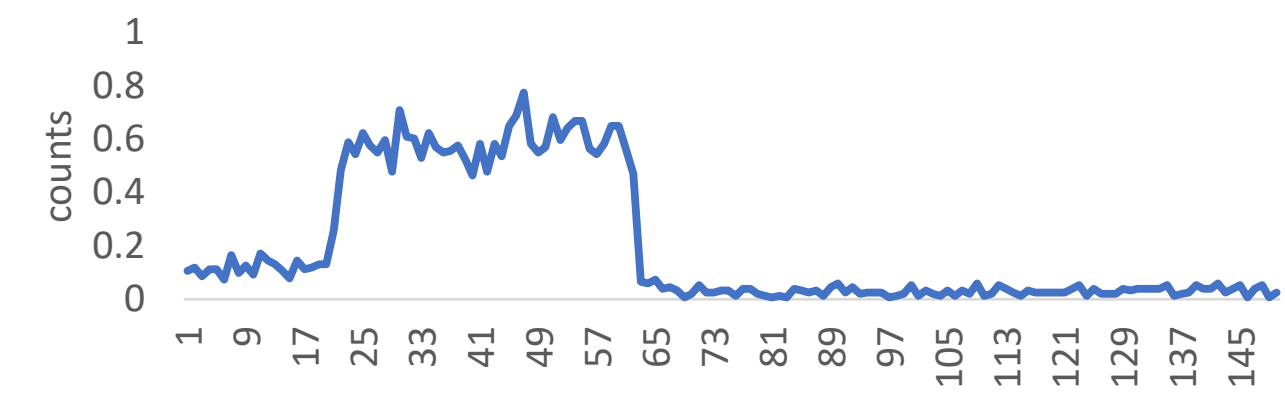

Frames

\section{Falling}

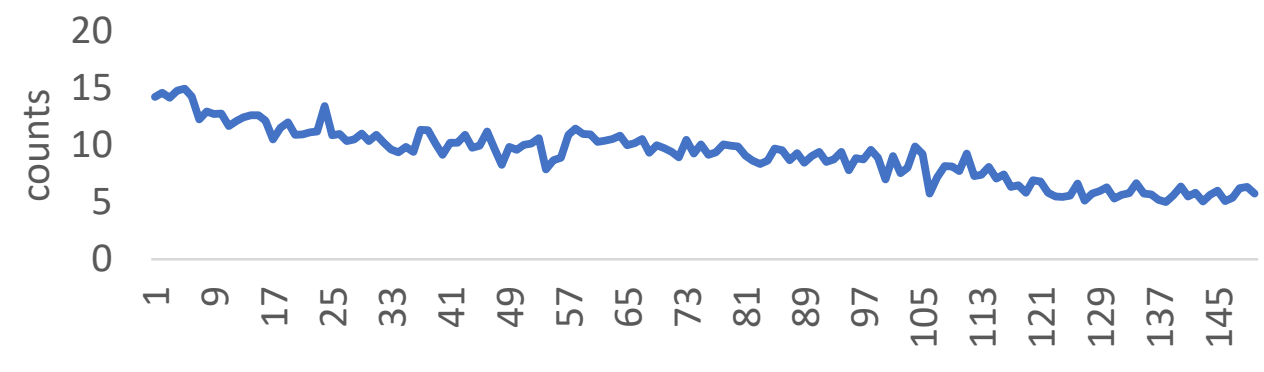

Frames

Figure 15: Representative reflux time-activity-curves (TAC) are depicted above. Reflux TAC are classified as one of the following; rising (reflecting repeated reflux events with failure of clearance), flat (no reflux or reflux events with return to baseline between events) or falling (reflux with delayed clearance). 
Figure 16

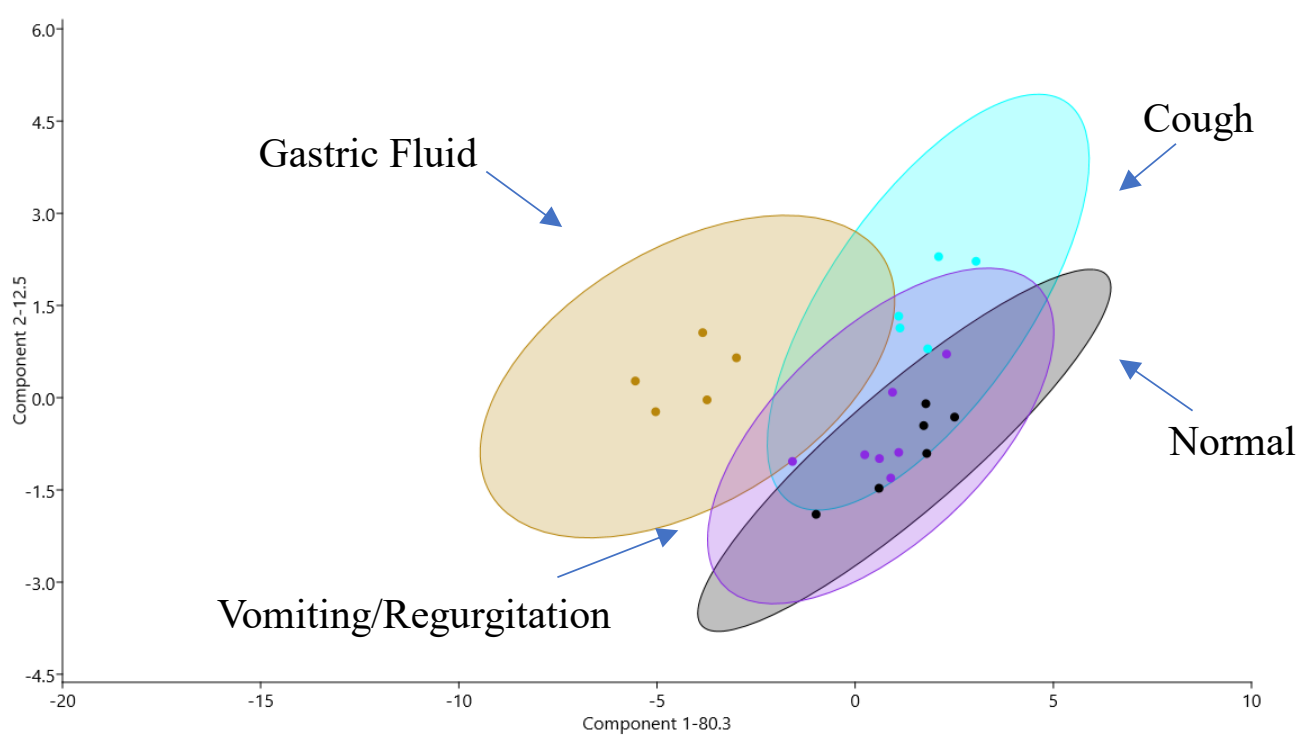

Figure 16: Proteomic profiles as shown via principal component (PC) analysis of samples for all 4 samples sites: GF (yellow), normal OP (gray), cough OP (blue), and vomiting/regurgitation OP (purple). Similarities in proteomic profiles are demonstrated by overlapping regions on the PC1 versus PC2 plot. The ellipses represent $95 \%$ intervals. Gastric fluid (GF), Oropharyngeal (OP). 
Figure 17

\begin{tabular}{|c|c|c|c|c|}
\hline & Gastric & Normal & Vomiting/Regurgitation & Coughing \\
\hline Gastric & & 0.0007 & 0.001 & 0.009 \\
\hline Normal & 0.0007 & & 0.0006 & 0.001 \\
\hline Vomiting/Regurgitation & 0.001 & 0.0006 & & 0.0009 \\
\hline Coughing & 0.009 & 0.001 & 0.0009 & \\
\hline \multicolumn{5}{|l|}{$100 \%$} \\
\hline \multicolumn{5}{|l|}{$90 \%$} \\
\hline \multicolumn{5}{|l|}{$80 \%$} \\
\hline $70 \%$ & & & & \\
\hline $60 \%$ & & & & \\
\hline \multicolumn{5}{|l|}{$50 \%$} \\
\hline \multicolumn{5}{|l|}{$40 \%$} \\
\hline \multicolumn{5}{|l|}{$30 \%$} \\
\hline \multicolumn{5}{|l|}{$20 \%$} \\
\hline \multicolumn{5}{|l|}{$10 \%$} \\
\hline \multicolumn{5}{|c|}{ T. $\quad . \quad$. } \\
\hline $\begin{array}{l}\text { Regulation of } \\
\text { Structural mol } \\
\text { Cell different } \\
\text { Unclassified }\end{array}$ & $\begin{array}{l}\text { ological } \\
\text { ule } \\
\text { on, orga }\end{array}$ & enesis & $\begin{array}{l}\text { mune } \\
\text { tabolic process } \\
\text { nsport } \\
\text { iponse to stimulus }\end{array}$ & \\
\hline
\end{tabular}

Figure 17: All groups were statistically dissimilar by ANOSIM based on biological function of identified proteins $(p \leq 0.001)$. This suggests the functional proteome differs by site (GF vs. OP) as well as between health and disease. The biological function profile for each group is displayed in the stacked bar chart above. Metabolic and immune proteins were over-represented in gastric fluid compared to normal dog OP swabs $(\mathrm{p}<0.05)$. Metabolic proteins were over-represented in vomiting/regurgitating dog OP swabs compared to healthy dogs $(p<0.05)$, as were proteins with immunologic function 
$(p<0.05)$. Coughing dog OP swabs had increased numbers of proteins involved cellular differentiation and stimulus response compared to normal and regurgitating/vomiting $\operatorname{dogs}(p<0.05)$ 
Figure 18

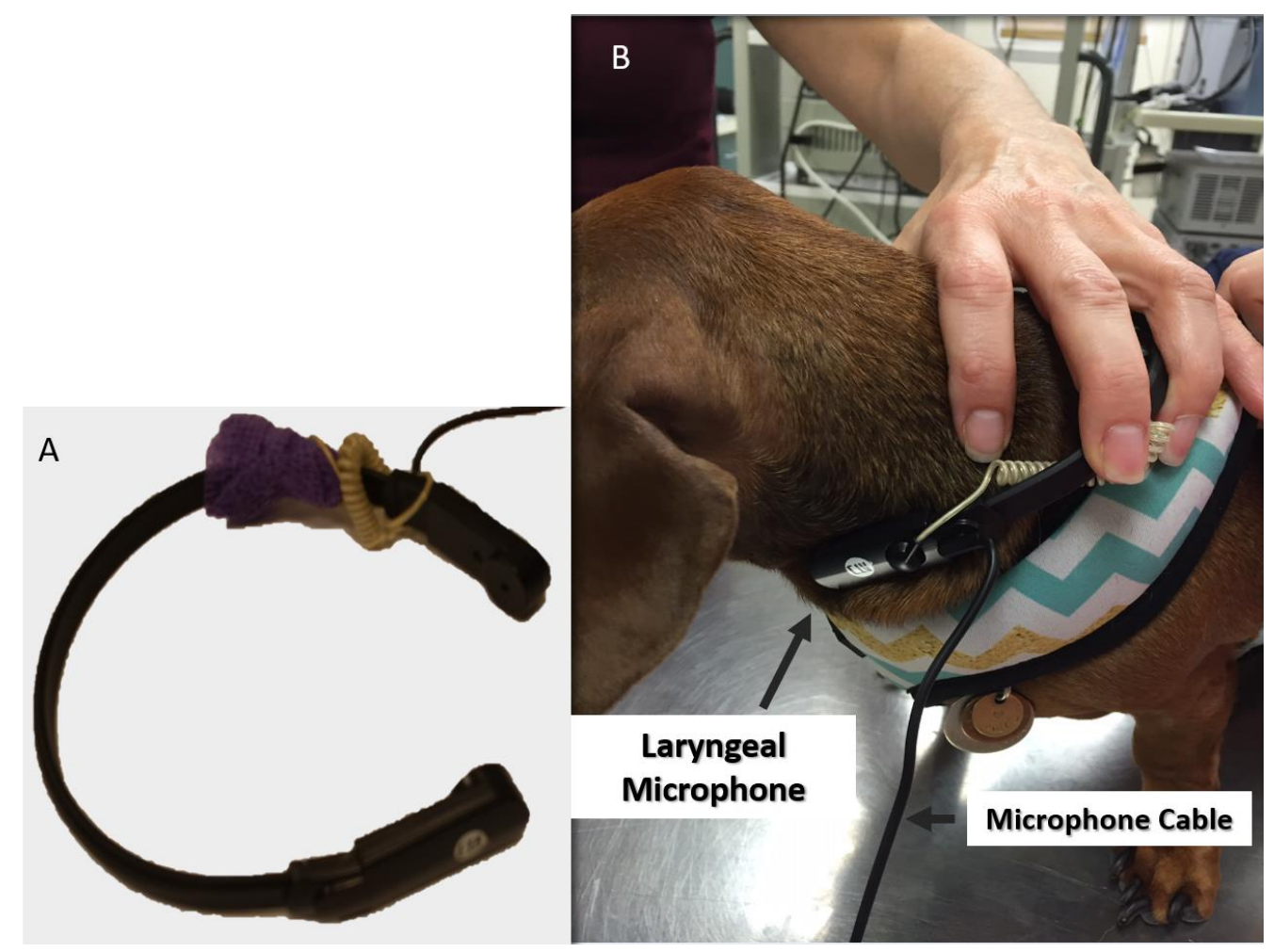

Figure 18: Representative positioning of a microphone at the level of the larynx (black arrow). The microphone was connected directly to a laptop computer equipped with bioacoustics collection and analysis software (RavenPro). Dogs were minimally restrained during data collection. 
Figure 19

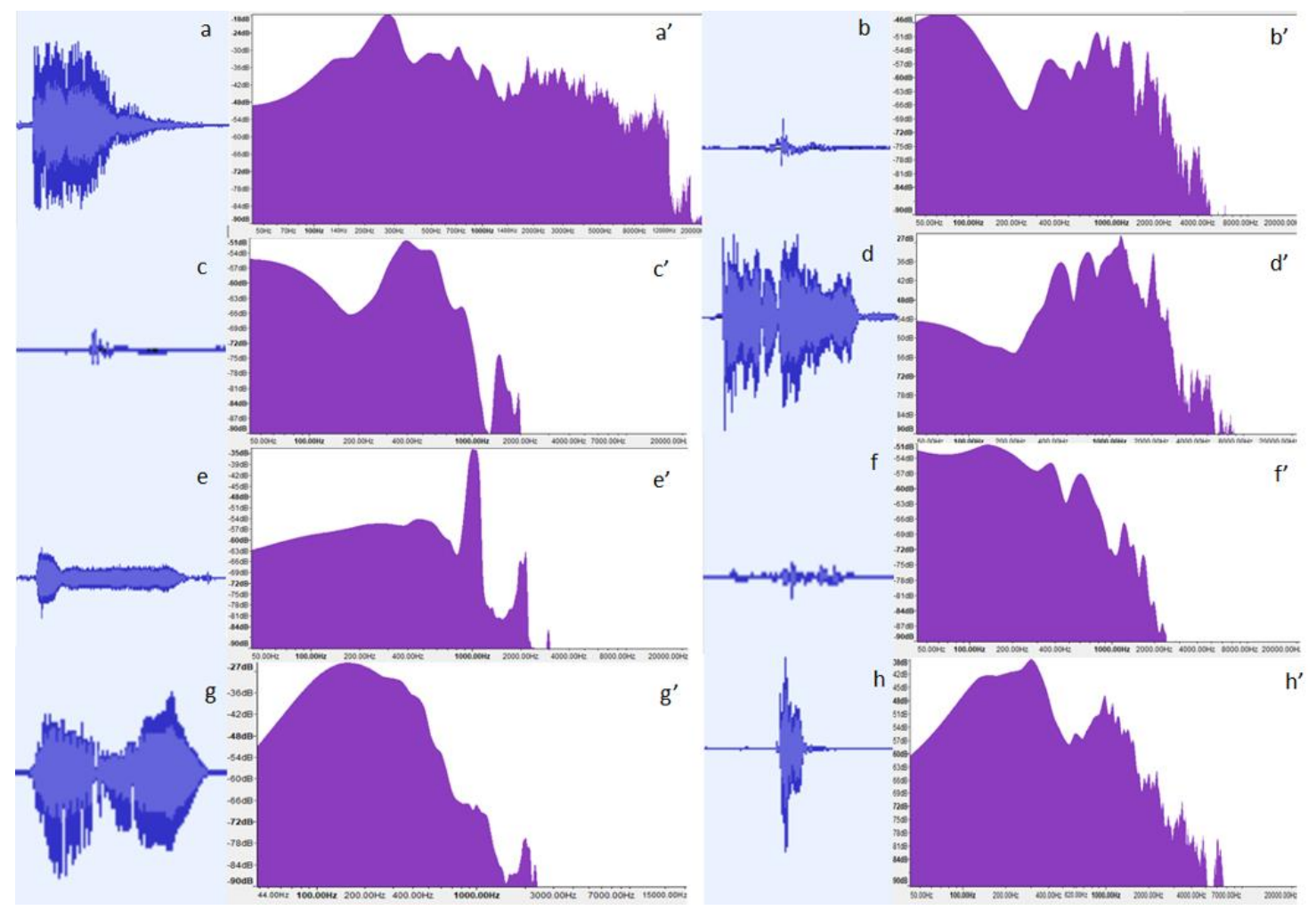

Figure 19a-h: Representative acoustic waveforms (a-h) and spectra (a'-h') are provided for a) cough b) chew c) lick d) bark e) whine f) drink g) growl h) throat-clear (TC). Waveforms describe amplitude over time. Median IQR waveform characteristics are provided in Table 2. Spectral plots show frequency on the $\mathrm{x}$ axis and $\mathrm{dB}$ on the $\mathrm{y}$ axis. 


\section{Tables}

Table 1

\begin{tabular}{|c|c|}
\hline Human & Dogs \\
\hline $\begin{array}{ll}\text { - } & \text { Laryngitis(314) } \\
\text { - } & \text { Laryngeal paralysis/dysfunction(28) } \\
\text { - } & \text { Large airway obstruction(28) } \\
\text { - } & \text { Bronchiectasis(315) } \\
\text { - } & \text { Diffuse aspiration bronchiolitis }(316) \\
\text { - } & \text { Aspiration pneumonia/pneumonitis }(28) \\
\text { - } & \text { Exogenous lipid pneumonia(317) } \\
\text { - } & \text { Interstitial lung disease(97) } \\
\text { - } & \text { Acute respiratory distress syndrome } \\
\text { - } & \text { Chronic cough(234) } \\
\text { - } & \text { Bronchiolitis obliterans syndrome }(83) \\
\text { - } & \text { Asthma(314) } \\
\text { - } & \text { Chronic obstructive pulmonary } \\
\text { - } & \text { diseases(314) } \\
\text { - } & \text { Otitis }(318)\end{array}$ & $\begin{array}{ll}\text { - } & \text { Laryngitis }(12,319) \\
& \text { Laryngeal paralysis/dysfunction(12, } \\
\text { - } & \text { Large airway obstruction(320) } \\
\text { - } & \text { Bronchiectasis }(152) \\
\text { - } & \text { Diffuse aspiration bronchiolitis }(12) \\
\text { - } & \text { Aspiration } \\
\text { - } & \text { pneumonia/pneumonitis }(12) \\
\text { - } & \text { Interstitial lung disease }(12,98) \\
\text { - } & \text { Acute respiratory distress } \\
& \text { syndrome }(12) \\
\text { - Otitis }(77)\end{array}$ \\
\hline
\end{tabular}

Table 1: Aspiration associated respiratory diseases reported in the human and veterinary

literature. 
Table 2

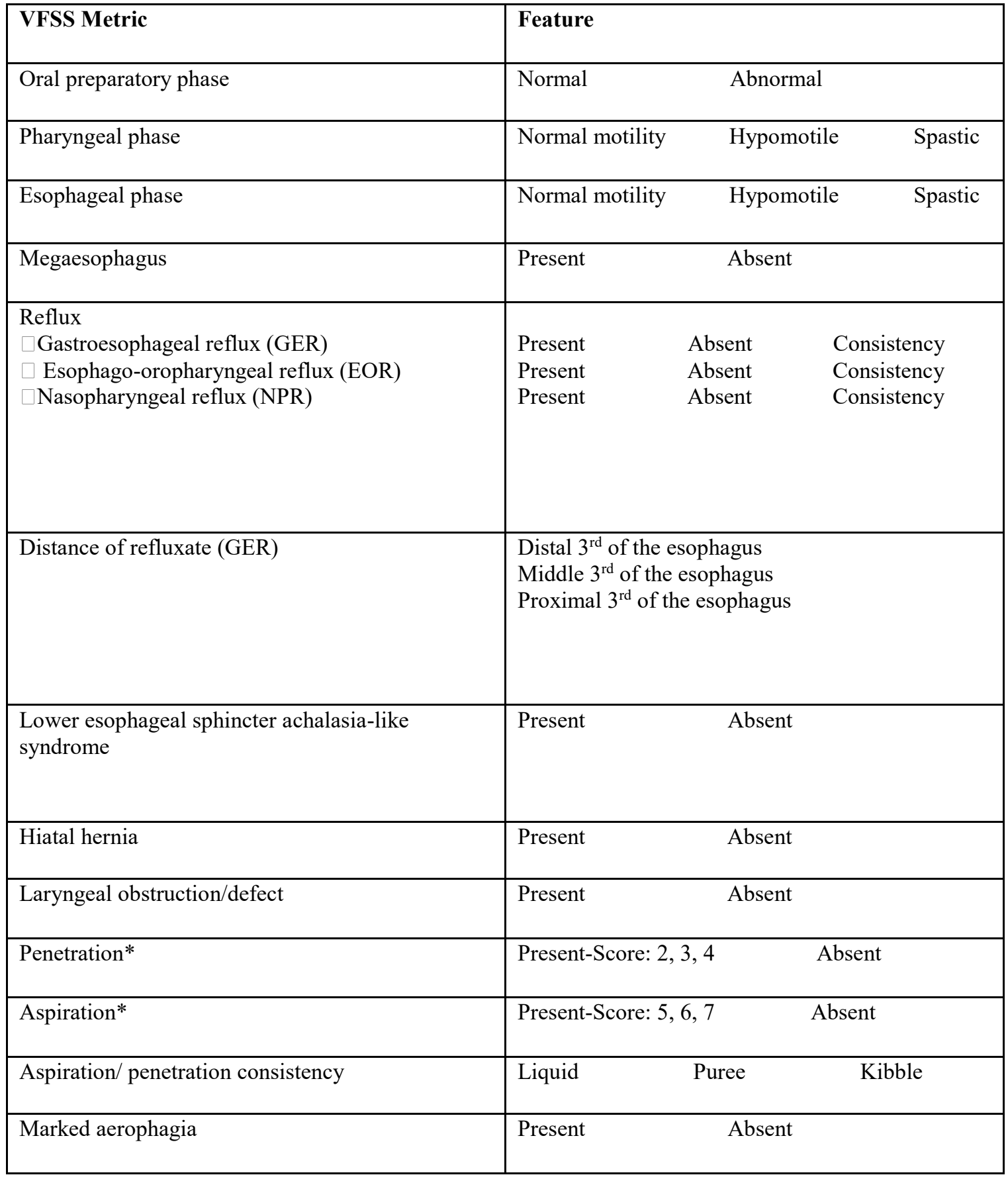

Table 2: Standardized VFSS scoring rubric for aerodigestive disorders in the dog. *

Penetration-Aspiration scoring system displayed in Table 4. Reflux extending past the middle $3^{\text {rd }}$ of the esophagus was considered pathologic. 
Table 3

\begin{tabular}{|c|c|}
\hline Criteria for evaluation & Definition \\
\hline \multicolumn{2}{|l|}{ Respiratory } \\
\hline $\begin{array}{l}\text { - Upper airway } \\
\circ \text { Airways proximal } \\
\text { to the thoracic } \\
\text { inlet }\end{array}$ & $\begin{array}{l}\text { Upper airway structures including the naso-, oro-, and } \\
\text { laryngopharynx as well as the cervical trachea were evaluated } \\
\text { for static or dynamic collapse during quiet breathing. }\end{array}$ \\
\hline $\begin{array}{ll}\text { - } & \text { Laryngeal } \\
\text { obstruction/defect (LO/D) }\end{array}$ & $\begin{array}{l}\text { Movement of the epiglottis with respect to swallow and } \\
\text { respiration was evaluated. The larynx was also evaluated for } \\
\text { appropriate rostral movement during pharyngeal swallow. } \\
\text { - Laryngeal defects were considered to contribute to } \\
\text { aerodigestive disease (AeroD) when there was } \\
\text { concurrent evidence of penetration, aspiration, } \\
\text { esophago-oropharyngeal reflux (EOR), or defect in } \\
\text { pharyngeal swallow. }\end{array}$ \\
\hline $\begin{array}{c}\text { - Lower airway } \\
\circ \quad \text { Airways distal to } \\
\text { the thoracic inlet }\end{array}$ & $\begin{array}{l}\text { The intrathoracic trachea and main-stem bronchi (MSB) were } \\
\text { evaluated for static or dynamic collapse/compression during } \\
\text { quiet breathing. }\end{array}$ \\
\hline $\begin{array}{l}\text { - Diaphragm and thoracic } \\
\text { cage }\end{array}$ & $\begin{array}{l}\text { Movement of the diaphragm and ribs were evaluated for } \\
\text { appropriate movement during quiet breathing. } \\
\text { - Diaphragm: Flattening on inspiration } \\
\text { - Ribs: Rostral movement on inspiration }\end{array}$ \\
\hline \multicolumn{2}{|l|}{ VFSS } \\
\hline $\begin{array}{l}\text { - Oral preparatory }(\mathrm{O}-\mathrm{P}) \\
\text { phase }\end{array}$ & $\begin{array}{l}\text { Dogs were assessed for appropriate jaw excursion, mastication, } \\
\text { and collection of the food bolus in the valleculae (between the } \\
\text { base of the tongue and the epiglottis). }\end{array}$ \\
\hline - $\quad$ Pharyngeal phase & $\begin{array}{l}\text { Dogs were assessed for appropriate pharyngeal constriction and } \\
\text { conduction of a bolus from the pharynx through the upper } \\
\text { esophageal sphincter (UES) into the proximal esophagus. } \\
\text { Greater than one swallow attempt per bolus or } \\
\text { pharyngeal bolus discohesion (incomplete bolus } \\
\text { clearance) for the majority of swallows was } \\
\text { considered abnormal. Residual contrast in the pharynx } \\
\text { during respiration was considered abnormal regardless } \\
\text { of the number of swallow attempts or the presence of } \\
\text { aspiration into the trachea. }\end{array}$ \\
\hline $\begin{array}{l}\text { - Esophageal contraction } \\
\text { and peristalsis }\end{array}$ & $\begin{array}{l}\text { These were defined as waves initiated by pharyngeal swallow, } \\
\text { beginning in the proximal esophagus and capable of } \\
\text { conducting the food bolus aborally towards the lower } \\
\text { esophageal sphincter (LES). }\end{array}$ \\
\hline
\end{tabular}




\begin{tabular}{|c|c|}
\hline - $\quad$ Megaesophagus (ME) & Subjective assessment of esophageal dilation \\
\hline $\begin{array}{l}\text { - Gastroesophageal } \\
\text { reflux (GER) }\end{array}$ & $\begin{array}{l}\text { Orad movement of contrast from the stomach into the } \\
\text { esophagus. } \\
\text { The maximal orad movement of contrast during GER } \\
\text { was recorded: proximal } 3^{\text {rd }} \text { of the esophagus, middle } \\
3^{\text {rd }} \text { of the esophagus, distal } 3^{\text {rd }} \text { of the esophagus. } \\
\text { Reflux extending past the middle } 3^{\text {rd }} \text { of the esophagus } \\
\text { was considered abnormal.(147) }\end{array}$ \\
\hline $\begin{array}{ll}\text { - } & \begin{array}{l}\text { Esophago- } \\
\text { oropharyngeal reflux } \\
\text { (EOR) }\end{array}\end{array}$ & $\begin{array}{l}\text { Orad movement of contrast from the esophagus into the } \\
\text { oropharynx. }\end{array}$ \\
\hline $\begin{array}{l}\text { - Nasopharyngeal reflux } \\
\text { (NPR) }\end{array}$ & $\begin{array}{l}\text { Movement of contrast from the pharynx to the nasopharynx } \\
\text { during pharyngeal swallow or with EOR. }\end{array}$ \\
\hline $\begin{array}{ll} & \text { Lower esophageal } \\
\text { sphincter achalasia-like } \\
\text { syndrome (LES-AS) }\end{array}$ & $\begin{array}{l}\text { Dogs were assessed for a failure of the LES to relax in } \\
\text { response to a pharyngeal swallow.(148) }\end{array}$ \\
\hline - Hiatal hernia $(\mathrm{HH})$ & $\begin{array}{l}\text { Dogs were assessed for herniation of the stomach into the } \\
\text { thoracic cavity through the esophageal hiatus either passively } \\
\text { or in response to abdominal pressure. } \\
\text { During abdominal compression, a licensed } \\
\text { veterinarian wearing appropriate personal protective } \\
\text { equipment applied abdominal pressure to a standing } \\
\text { dog in order to induce a sliding hiatal hernia. }\end{array}$ \\
\hline $\begin{array}{l}\text { - Penetration-Aspiration (A- } \\
\text { P) }\end{array}$ & $\begin{array}{l}\text { Penetration: Material enters the airway but remains above the } \\
\text { vocal folds } \\
\text { Aspiration: Material enters the airway and extends past the } \\
\text { vocal folds }\end{array}$ \\
\hline - $\quad$ Aerophagia & $\begin{array}{l}\text { This was defined as the swallowing of substantial volumes of } \\
\text { air. Aerophagia was considered "marked" if gas comprised }> \\
1 / 3 \text { of the bolus volume and/or resulted in gastric distention (air } \\
\text { accounting for }>1 / 3 \text { of the end gastric volume). }\end{array}$ \\
\hline
\end{tabular}

Table 3: Standardized criteria for evaluation for respiratory fluoroscopy and

videofluoroscopic swallow studies. Penetration-Aspiration scale is available in Table 4. 
Table 4

\begin{tabular}{|l|l|l|}
\hline Classification & Score & Description \\
\hline Normal & 1 & Material does not enter the airway \\
\hline Penetration & 2 & $\begin{array}{l}\text { Material is in the supraepiglottic space, remains above the } \\
\text { vocal folds but leaves the airway before epiglottis returns } \\
\text { to rest position }\end{array}$ \\
\hline & 3 & $\begin{array}{l}\text { Material is in the supraepiglottic space, remains above the } \\
\text { vocal folds after epiglottis has returned to rest position }\end{array}$ \\
\hline Aspiration & 4 & $\begin{array}{l}\text { Material is in the supraepiglottic space, large amount } \\
\text { remains in above the vocal folds after epiglottis returns to } \\
\text { rest position }\end{array}$ \\
\hline & 5 & $\begin{array}{l}\text { Material passes bellow the vocal folds, and is actively } \\
\text { ejected }\end{array}$ \\
\hline & 7 & $\begin{array}{l}\text { Material passes bellow the vocal folds and is not ejected } \\
\text { despite effort }\end{array}$ \\
\hline
\end{tabular}

Table 4: Penetration-Aspiration Scale(322) describing entrance of liquid or food into the larynx and trachea. (Figure 3) 
Table 5

\begin{tabular}{|c|c|}
\hline Respiratory Diagnosis & Diagnostic Criteria \\
\hline Canine chronic bronchitis (CCB) & $\begin{array}{c}\geq 10 \% \text { non-degenerate neutrophils } \pm \geq 10 \% \\
\text { eosinophils on bronchoalveolar lavage fluid } \\
\text { (BALF) cytology }\end{array}$ \\
\hline Eosinophilic bronchitis (EB) & $\geq 10 \%$ eosinophils on BALF cytology \\
\hline Radiographic evidence of aspiration pneumonia (AP) & $\begin{array}{c}\text { Interstitial-alveolar pattern in dependent lung } \\
\text { regions. A secondary bacterial infection was } \\
\text { determined by the presence of intracellular } \\
\text { bacteria noted on BALF cytology and/or a } \\
\text { positive BALF culture }\end{array}$ \\
\hline Laryngeal paralysis (LarPar) & $\begin{array}{c}\text { The absence of laryngeal abduction on } \\
\text { inspiration after treatment with doxopram }\end{array}$ \\
\hline Epiglottic retroversion (ER) & $\begin{array}{c}\text { Intermittent retroversion of the epiglottis on } \\
\text { inspiration resulting in intermittent obstruction } \\
\text { of the rima glottidis }\end{array}$ \\
\hline Bronchomalacia (BM) & $\begin{array}{c}\text { Static or dynamic collapse of the airways at the } \\
\text { level of or distal to the principle bronchi by } \\
>50 \%\end{array}$ \\
\hline Bronchiectasis (BE) & $\begin{array}{c}\text { Architectural remodeling resulting in airway } \\
\text { dilation with failure of tapering of the lumen } \\
\text { with distal progression }\end{array}$ \\
\hline Hypoplastic trachea & $\begin{array}{c}\text { Fixed narrowing of the trachea with a ratio of } \\
\text { trachea to the }{ }^{\text {rd }} \text { rib of }<2.0\end{array}$ \\
\hline
\end{tabular}

Table 5: Diagnostic criteria for specific respiratory diagnosis. 
Table 6

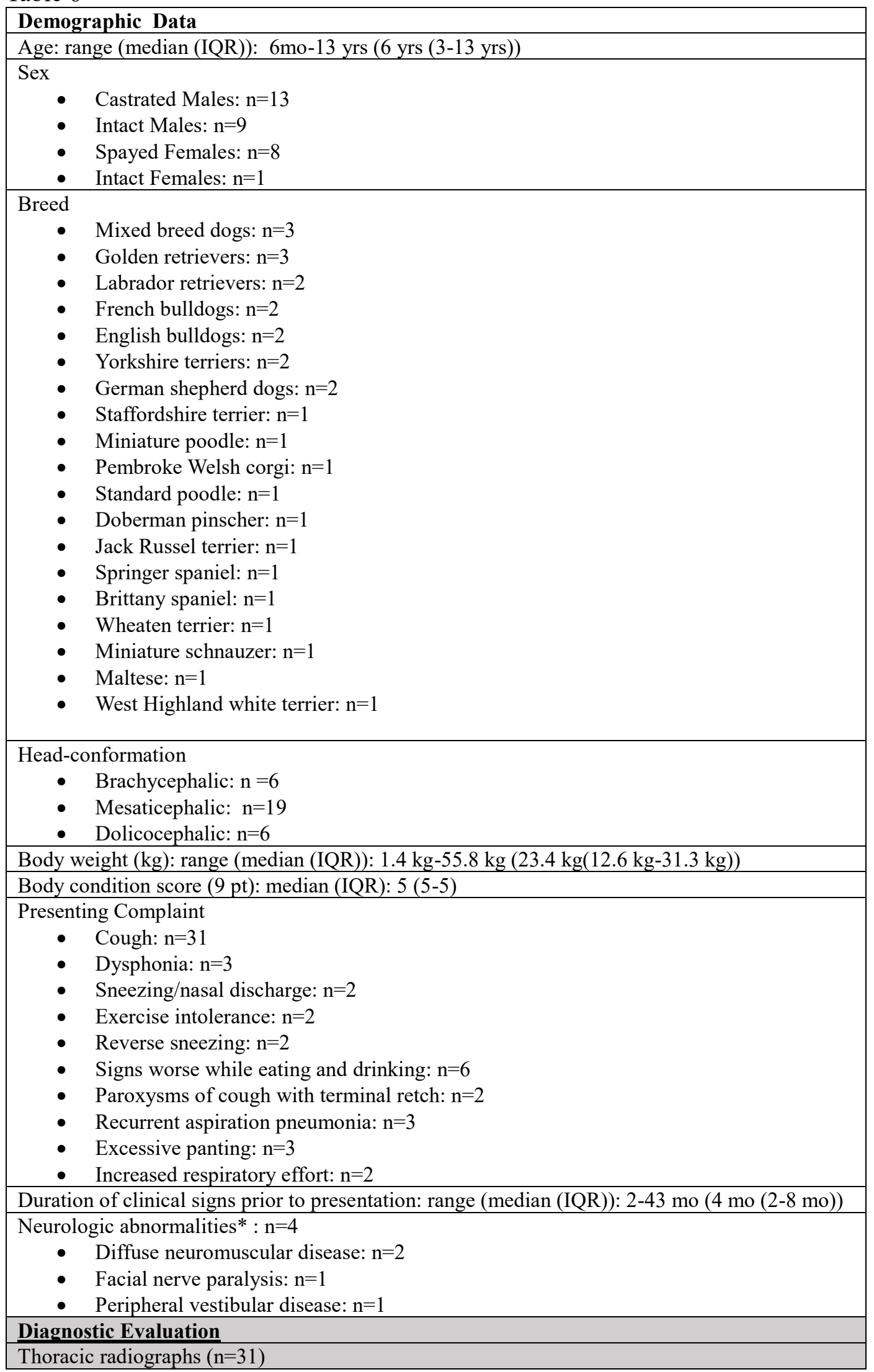


- Unremarkable: $\mathrm{n}=11$

- Diffuse bronchial pattern: $\mathrm{n}=11$

- Radiographic evidence of aspiration pneumonia : $\mathrm{n}=7$

- Other

- ME: $\mathrm{n}=3$

- Tracheal and main-stem bronchial collapse: $\mathrm{n}=1$

- Hypoplastic trachea: $n=1$

- Bronchiectasis: $\mathrm{n}=1$

- Diffuse pulmonary osteomas: $\mathrm{n}=1$

- Cardiomegaly: $n=1$

Laryngeal function examination ( $\mathrm{n}=15$ : under propofol; respiration stimulated with doxapram)

- Erythema: $\mathrm{n}=12$

- Laryngeal paralysis: $\mathrm{n}=7$

- Unremarkable: $\mathrm{n}=3$

- Laryngeal edema/swelling: $\mathrm{n}=1$

- Epiglottic retroversion: $\mathrm{n}=1$

- Laryngeal polyp: $\mathrm{n}=1$

Tracheobronchoscopy $(\mathrm{n}=13)$

- Diffuse erythema: $\mathrm{n}=7$

- Bronchiectasis: $\mathrm{n}=5$

- Bronchomalacia: $\mathrm{n}=5$

- Tenacious mucous: $\mathrm{n}=4$

- Increased tracheal vascularity: $\mathrm{n}=1$

- Tracheal stripe** $\mathrm{w}: \mathrm{n}=1$

BALF cytology $(\mathrm{n}=13)$

- Neutrophilic: $\mathrm{n}=6$

○ Non-septic: $\mathrm{n}=4$

- Septic: $n=2$

- Eosinophilic: $\mathrm{n}=2$

- Mixed inflammation: $\mathrm{n}=3$

- Unremarkable: $\mathrm{n}=2$

BALF culture $(n=13)$

- Negative: $\mathrm{n}=11$

- Positive: $\mathrm{n}=2$

- N=1: Beta hemolytic Streptococcus spp, Klebsiella oxytoca, E.coli, and Salmonella typhimuirium

- N=2: Pseudomonas aeruginosa

Table 6: Demographic (white) and diagnostic (grey) data from $\mathrm{n}=31 \mathrm{dogs}$ presenting for

cough in the absence of gastrointestinal signs. * Advanced diagnostics including MRI and electromyography (EMG) were not performed in dogs with neurologic disease. **

Tracheal stripe: proximal erythema of the trachea that dissipates toward the carina.

(Figure 2) 
Table 7

\begin{tabular}{|c|c|c|c|c|c|c|c|c|c|c|c|c|c|c|c|}
\hline Dog & $\begin{array}{c}\text { Respiratory } \\
\text { Dx }\end{array}$ & $\begin{array}{l}\text { VFSS } \\
\text { Dx }\end{array}$ & Loc. & OP & $\begin{array}{c}\text { P- } \\
\text { Cont }\end{array}$ & LO/D & $\overline{\mathbf{A}}$ & $\mathbf{P}$ & NPR & ME & E-PP & $\begin{array}{c}\text { LES- } \\
\text { AS }\end{array}$ & Ref & HH & Aero \\
\hline 1 & None & None & None & $\mathrm{N}$ & Norm & $\mathrm{N}$ & $\mathrm{N}$ & $\mathrm{N}$ & $\mathrm{N}$ & $\mathrm{N}$ & Norm & $\mathrm{N}$ & $\mathrm{N}$ & $\mathrm{N}$ & $\mathrm{N}$ \\
\hline & & & & & & & & & & & & & & & \\
\hline 2 & None & None & None & $\mathrm{N}$ & Norm & $\mathrm{N}$ & $\mathrm{N}$ & $\mathrm{N}$ & $\mathrm{N}$ & $\mathrm{N}$ & Norm & $\mathrm{N}$ & $\mathrm{N}$ & $\mathrm{N}$ & $\mathrm{N}$ \\
\hline 3 & $\mathrm{CCB}$ & None & Airway & $\mathrm{N}$ & Norm & $\mathrm{N}$ & $\mathrm{N}$ & $\mathrm{N}$ & $\mathrm{N}$ & $\mathrm{N}$ & Norm & $\mathrm{N}$ & $\mathrm{N}$ & $\mathrm{N}$ & $\mathrm{N}$ \\
\hline 4 & BE, BM & None & Airway & $\mathrm{N}$ & Norm & $\mathrm{N}$ & $\mathrm{N}$ & $\mathrm{N}$ & $\mathrm{N}$ & $\mathrm{N}$ & Norm & $\mathrm{N}$ & $\mathrm{N}$ & $\mathrm{N}$ & $\mathrm{N}$ \\
\hline 5 & EB & None & Airway & $\mathrm{N}$ & Norm & $\mathrm{N}$ & $\mathrm{N}$ & $\mathrm{N}$ & $\mathrm{N}$ & $\mathrm{N}$ & Norm & $\mathrm{N}$ & $\mathrm{N}$ & $\mathrm{N}$ & $\mathrm{N}$ \\
\hline 6 & $\begin{array}{l}\text { AP(bact), } \\
\text { LarPar }\end{array}$ & None & PP, Lar & $\mathrm{N}$ & Norm & $\mathrm{N}$ & $\mathrm{N}$ & $\mathrm{N}$ & $\mathrm{N}$ & $\mathrm{N}$ & Norm & $\mathrm{N}$ & $\mathrm{N}$ & $\mathrm{N}$ & $\mathrm{N}$ \\
\hline 7 & None & EH & Ali & $\mathrm{N}$ & Norm & $\mathrm{N}$ & $\mathrm{N}$ & $\mathrm{N}$ & $\mathrm{N}$ & $\mathrm{N}$ & Norm & $\mathrm{N}$ & $\mathrm{N}$ & $\mathrm{N}$ & $\mathrm{N}$ \\
\hline 8 & None & $\begin{array}{l}\text { EH, ME, } \\
\text { LES-AS }\end{array}$ & Ali & $\mathrm{N}$ & Norm & $\mathrm{N}$ & $\mathrm{N}$ & $\mathrm{N}$ & $\mathrm{N}$ & $\bar{Y}$ & EH & $\mathrm{Y}$ & $\mathrm{N}$ & $\mathrm{N}$ & $\mathrm{N}$ \\
\hline 9 & None & $\begin{array}{l}\text { OP, PH, } \\
\text { P, NPR }\end{array}$ & Ali & $\mathrm{Y}$ & $\mathrm{PH}$ & $\mathrm{N}$ & $\mathrm{N}$ & $\mathrm{Y}$ & $\mathrm{Y}$ & $\mathrm{N}$ & Norm & $\mathrm{N}$ & $\mathrm{N}$ & $\mathrm{N}$ & $\mathrm{N}$ \\
\hline 10 & None & $\begin{array}{l}\text { OP, PS, } \\
\text { P, EH }\end{array}$ & Ali & $\mathrm{Y}$ & PS & $\mathrm{N}$ & $\mathrm{N}$ & $\mathrm{Y}$ & $\mathrm{N}$ & $\mathrm{N}$ & $\mathrm{EH}$ & $\mathrm{N}$ & $\mathrm{N}$ & $\mathrm{N}$ & $\mathrm{N}$ \\
\hline 11 & None & OP, Ref & Ali & $\mathrm{Y}$ & Norm & $\mathrm{N}$ & $\mathrm{N}$ & $\mathrm{N}$ & $\mathrm{N}$ & $\mathrm{N}$ & Norm & $\mathrm{N}$ & $\mathrm{Y}$ & $\mathrm{N}$ & $\mathrm{N}$ \\
\hline 12 & None & $\mathrm{PH}$ & Ali & $\mathrm{N}$ & PH & $\mathrm{N}$ & $\mathrm{N}$ & $\mathrm{N}$ & $\mathrm{N}$ & $\mathrm{N}$ & Norm & $\mathrm{N}$ & $\mathrm{N}$ & $\mathrm{N}$ & $\mathrm{N}$ \\
\hline 13 & None & $\mathrm{PH}, \mathrm{EH}$ & Ali & $\mathrm{N}$ & PH & $\mathrm{N}$ & $\mathrm{N}$ & $\mathrm{N}$ & $\mathrm{N}$ & $\mathrm{N}$ & EH & $\mathrm{N}$ & $\mathrm{N}$ & $\mathrm{N}$ & $\mathrm{N}$ \\
\hline 14 & None & Ref & Ali & $\mathrm{N}$ & Norm & $\mathrm{N}$ & $\mathrm{N}$ & $\mathrm{N}$ & $\mathrm{N}$ & $\mathrm{N}$ & Norm & $\mathrm{N}$ & $\mathrm{Y}$ & $\mathrm{N}$ & $\mathrm{N}$ \\
\hline 15 & $\begin{array}{l}\text { Airway collapse } \\
\text { (Trachea/MSB) }\end{array}$ & Aero & Airway & $\mathrm{N}$ & Norm & $\mathrm{N}$ & $\mathrm{N}$ & $\mathrm{N}$ & $\mathrm{N}$ & $\mathrm{N}$ & Norm & $\mathrm{N}$ & $\mathrm{N}$ & $\mathrm{N}$ & $\bar{Y}$ \\
\hline 16 & $\mathrm{CCB}, \mathrm{BM}$ & $\begin{array}{l}\mathrm{EH}, \mathrm{P}, \\
\mathrm{PH}\end{array}$ & Airway & $\mathrm{N}$ & PH & $\mathrm{N}$ & $\mathrm{N}$ & $\mathrm{Y}$ & $\mathrm{N}$ & $\mathrm{N}$ & $\mathrm{EH}$ & $\mathrm{N}$ & $\mathrm{N}$ & $\mathrm{N}$ & $\mathrm{N}$ \\
\hline 17 & CCB & PH & Airway & $\mathrm{N}$ & $\mathrm{PH}$ & $\mathrm{N}$ & $\mathrm{N}$ & $\mathrm{N}$ & $\mathrm{N}$ & $\mathrm{N}$ & Norm & $\mathrm{N}$ & $\mathrm{N}$ & $\mathrm{N}$ & $\mathrm{N}$ \\
\hline 18 & CCB, LarPar & HH, Ref, & Airway, Lar & $\mathrm{N}$ & Norm & $\mathrm{N}$ & $\mathrm{N}$ & $\mathrm{N}$ & $\mathrm{N}$ & $\mathrm{N}$ & Norm & $\mathrm{N}$ & $\mathrm{Y}$ & $\mathrm{Y}$ & $\mathrm{N}$ \\
\hline 19 & $\begin{array}{l}\text { CCB, LarPar, } \\
\text { BM }\end{array}$ & $\begin{array}{l}\text { OP, P, } \\
\text { Ref, PH }\end{array}$ & Airway, Lar & $\mathrm{Y}$ & $\mathrm{PH}$ & $\mathrm{N}$ & $\mathrm{Y}$ & $\bar{Y}$ & $\mathrm{~N}$ & $\mathrm{~N}$ & Norm & $\mathrm{N}$ & $\mathrm{Y}$ & $\mathrm{N}$ & $\mathrm{N}$ \\
\hline 20 & EB, ER & $\mathrm{PH}, \mathrm{LO} / \mathrm{D}$ & Airway, Lar & $\mathrm{N}$ & $\mathrm{PH}$ & $\mathrm{Y}$ & $\mathrm{N}$ & $\mathrm{N}$ & $\mathrm{N}$ & $\mathrm{N}$ & Norm & $\mathrm{N}$ & $\mathrm{N}$ & $\mathrm{N}$ & $\mathrm{N}$ \\
\hline 21 & $\begin{array}{l}\text { LarPar, BE, } \\
\text { BM }\end{array}$ & Ref, EH & Airway, Lar & $\mathrm{N}$ & Norm & $\mathrm{N}$ & $\mathrm{N}$ & $\mathrm{N}$ & $\mathrm{N}$ & $\mathrm{N}$ & $\mathrm{EH}$ & $\mathrm{N}$ & $\bar{Y}$ & $\mathrm{~N}$ & $\mathrm{~N}$ \\
\hline 22 & $\begin{array}{l}\text { CCB, LarPar, } \\
\text { BE, BM }\end{array}$ & Aero & Airway, Lar & $\mathrm{N}$ & Norm & $\mathrm{N}$ & $\mathrm{N}$ & $\mathrm{N}$ & $\mathrm{N}$ & $\mathrm{N}$ & Norm & $\mathrm{N}$ & $\mathrm{N}$ & $\mathrm{N}$ & $\mathrm{Y}$ \\
\hline 23 & LarPar & $\begin{array}{l}\text { A, P, } \\
\text { PH, OP }\end{array}$ & Lar & $\mathrm{Y}$ & PH & $\mathrm{N}$ & $\mathrm{Y}$ & $\mathrm{Y}$ & $\mathrm{N}$ & $\mathrm{N}$ & Norm & $\mathrm{N}$ & $\mathrm{N}$ & $\mathrm{N}$ & $\mathrm{N}$ \\
\hline 24 & Lar. polyp & $\begin{array}{l}\text { Ref, A, } \\
\text { LO/D }\end{array}$ & Lar & $\mathrm{N}$ & Norm & $\mathrm{Y}$ & $\mathrm{Y}^{*}$ & $\mathrm{~N}$ & $\mathrm{~N}$ & $\mathrm{~N}$ & Norm & $\mathrm{N}$ & $\mathrm{Y}$ & $\mathrm{N}$ & $\mathrm{N}$ \\
\hline 25 & $\mathrm{AP}(\mathrm{rad})$ & $\begin{array}{l}\text { Aero, ME, } \\
\text { EH }\end{array}$ & PP & $\mathrm{N}$ & Norm & $\mathrm{N}$ & $\mathrm{N}$ & $\mathrm{N}$ & $\mathrm{N}$ & $\mathrm{Y}$ & $\mathrm{EH}$ & $\mathrm{N}$ & $\mathrm{N}$ & $\mathrm{N}$ & $\mathrm{Y}$ \\
\hline 26 & $\mathrm{AP}(\mathrm{rad})$ & $\begin{array}{l}\text { Aero, Ref, } \\
\text { EH, }\end{array}$ & PP & $\mathrm{N}$ & Norm & $\mathrm{N}$ & $\mathrm{N}$ & $\mathrm{N}$ & $\mathrm{N}$ & $\mathrm{N}$ & $\mathrm{EH}$ & $\mathrm{N}$ & $\mathrm{Y}$ & $\mathrm{N}$ & $\mathrm{Y}$ \\
\hline 27 & $\begin{array}{l}\text { AP(bact), } \\
\mathrm{BE}, \mathrm{BM}\end{array}$ & $\begin{array}{l}\text { Aero, Ref, } \\
\text { PH, HH }\end{array}$ & PP & $\mathrm{N}$ & PH & $\mathrm{N}$ & $\mathrm{N}$ & $\mathrm{N}$ & $\mathrm{N}$ & $\mathrm{N}$ & Norm & $\mathrm{N}$ & $\mathrm{Y}$ & $\mathrm{Y}$ & $\mathrm{Y}$ \\
\hline 28 & $\mathrm{AP}(\mathrm{rad}), \mathrm{ER}$ & $\mathrm{OP}, \mathrm{LO} / \mathrm{D}$ & PP & $\mathrm{Y}$ & Norm & $\mathrm{Y}$ & $\mathrm{N}$ & $\mathrm{N}$ & $\mathrm{N}$ & $\mathrm{N}$ & Norm & $\mathrm{N}$ & $\mathrm{N}$ & $\mathrm{N}$ & $\mathrm{N}$ \\
\hline 29 & $\begin{array}{l}\text { AP(rad), } \\
\text { HT }\end{array}$ & $\mathrm{EH}$ & PP, Airway & $\mathrm{N}$ & Norm & $\mathrm{N}$ & $\mathrm{N}$ & $\mathrm{N}$ & $\mathrm{N}$ & $\mathrm{N}$ & EH & $\mathrm{N}$ & $\mathrm{N}$ & $\mathrm{N}$ & $\mathrm{N}$ \\
\hline 30 & $\begin{array}{l}\text { AP(sterile), } \\
\mathrm{BE}\end{array}$ & $\begin{array}{l}\text { Ref, ME, } \\
\mathrm{EH}, \mathrm{MG}\end{array}$ & PP, Airway & $\mathrm{N}$ & Norm & $\mathrm{N}$ & $\mathrm{N}$ & $\mathrm{N}$ & $\mathrm{N}$ & $\mathrm{Y}$ & EH & $\mathrm{N}$ & $\mathrm{Y}$ & $\mathrm{N}$ & $\mathrm{N}$ \\
\hline 31 & $\begin{array}{l}\text { AP(rad), } \\
\text { LarPar }\end{array}$ & Aero, PH & PP. Lar & $\mathrm{N}$ & $\mathrm{PH}$ & $\mathrm{N}$ & $\mathrm{N}$ & $\mathrm{N}$ & $\mathrm{N}$ & $\mathrm{N}$ & Norm & $\mathrm{N}$ & $\mathrm{N}$ & $\mathrm{N}$ & $\mathrm{Y}$ \\
\hline
\end{tabular}

Table 7: Videofluoroscopic swallow study (VFSS) and respiratory abnormalities for all dogs. Dogs 1-2 (grey): No diagnosis was made despite standard respiratory workup and VFSS. Dogs 3-6 (white): A respiratory diagnosis was made with no concurrent alimentary abnormalities detected on VFSS. Dogs 7-14 (grey): Dogs were determined to have non-respiratory (alimentary cough) based on VFSS abnormalities and the absence of a concurrent respiratory diagnosis. Dogs 15-31 (white): Dogs had a respiratory source of cough in addition to VFSS abnormalities.

* aspiration with both puree and liquid

Respiratory Diagnoses: $(\mathrm{AP}(\mathrm{rad}))$ : radiographic dx of AP, (AP (bact)): radiographic 
evidence of AP with confirmed secondary bacterial infection, (AP (sterile)): radiographic evidence of AP without a secondary bacterial infection, (LarPar): laryngeal paralysis, (EB): eosinophilic bronchitis, (CCB): canine chronic bronchitis, (MSB): mainstem bronchi, (BE): bronchiectasis, (BM): bronchomalacia, (ER): epiglottic retroversion VFSS Criteria: (OP): oropharyngeal defect, (P-cont): pharyngeal contraction, (LO/D): laryngeal obstruction/defect, (A): aspiration, (P): penetration, (NPR): nasopharyngeal reflux, (ME): megaesophagus, (E-P): esophageal peristalsis, (LES-AS): lower esophageal sphincter achalasia-like syndrome, (Ref): reflux, (HH): hiatal hernia, (Aero): aerophagia, (Norm): normal VFSS Diagnoses: (Y): yes, (N): no, (PH): pharyngeal hypomotility, (PS): pharyngeal spasticity, (EH): esophageal hypomotility Disease localization: (Lar): laryngeal, (PP): pulmonary parenchymal 
Table 8

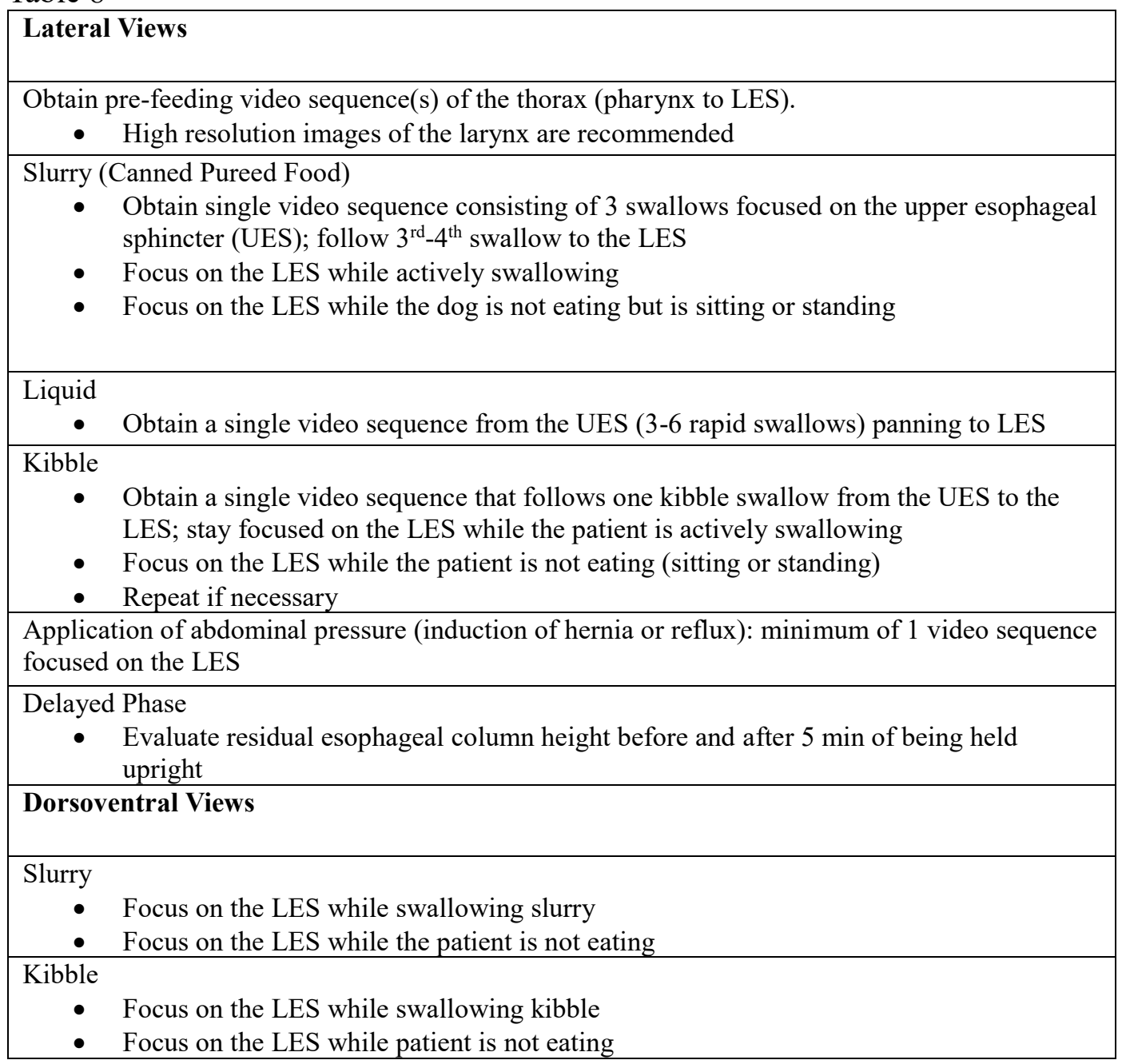

Table 8: Recommended minimum VFSS clips (video sequences) for a diagnosis of lower

esophageal achalasia-like syndrome in dogs. 
Table 9

\begin{tabular}{|c|c|}
\hline+2 & Feature \\
\hline Baseline fluid line & $\begin{array}{l}\text { Present } \\
\text { Absent }\end{array}$ \\
\hline Subjective ME & $\begin{array}{l}\text { Present } \\
\text { Absent }\end{array}$ \\
\hline Primary peristalsis (Contraction) & $\begin{array}{l}\text { Acontractile } \\
\text { Hypomotile } \\
\text { Hypermotile } \\
\text { Normal }\end{array}$ \\
\hline Primary peristalsis (Propulsion) & $\begin{array}{l}\text { Effective Complete } \\
\text { Effective Partial } \\
\text { Ineffective } \\
\text { Absent }\end{array}$ \\
\hline Primary peristaltic defect (Location) & $\begin{array}{l}\text { Focal } \\
\text { Diffuse }\end{array}$ \\
\hline Secondary peristalsis (Contraction) & $\begin{array}{l}\text { Acontractile } \\
\text { Hypomotile } \\
\text { Hypermotile } \\
\text { Normal }\end{array}$ \\
\hline Secondary peristalsis (Propulsion) & $\begin{array}{l}\text { Effective Complete } \\
\text { Effective Partial } \\
\text { Ineffective } \\
\text { Absent }\end{array}$ \\
\hline LES “bird beak" & $\begin{array}{l}\text { Present } \\
\text { Absent }\end{array}$ \\
\hline Narrowed/hypermotile distal esophagus & $\begin{array}{l}\text { Present } \\
\text { Absent }\end{array}$ \\
\hline Entry of ingesta into the stomach & $\begin{array}{l}\text { With pharyngeal swallow } \\
\text { With hydrostatic pressure } \\
\text { Complete obstruction } \\
\text { Normal }\end{array}$ \\
\hline Reflux & $\begin{array}{l}\text { Present } \\
\text { Absent }\end{array}$ \\
\hline Hiatal hernia & $\begin{array}{l}\text { Present } \\
\text { Absent }\end{array}$ \\
\hline Functional LES obstruction & $\begin{array}{l}\text { Present } \\
\text { Absent }\end{array}$ \\
\hline Consistency of food where achalasia was observed & $\begin{array}{l}\text { Puree } \\
\text { Liquid } \\
\text { Kibble }\end{array}$ \\
\hline Inappropriate LES function & $\begin{array}{l}>50 \% \text { of swallows } \\
<50 \% \text { of swallows }\end{array}$ \\
\hline Collective impression to support LES-AS & $\begin{array}{l}\text { Present } \\
\text { Absent }\end{array}$ \\
\hline Subtype & $\begin{array}{l}\text { Type } 1 \\
\text { Type } 2 \\
\text { Type } 3 \\
\end{array}$ \\
\hline
\end{tabular}


\begin{tabular}{|l|l|}
\hline & Pseudoachalasia \\
\hline
\end{tabular}

Table 9: Standardized VFSS scoring rubric for LES-achalasia like-syndrome in the dog 
Table 10

\begin{tabular}{|l|l|l|}
\hline \multicolumn{1}{|c|}{ VFSS Parameter } & \multicolumn{1}{c|}{ LES-AS } & \multicolumn{1}{c|}{ Control } \\
\hline $\begin{array}{l}\text { Failure of LES to open during } \\
\text { pharyngeal swallowing }\end{array}$ & $19 / 19$ & $0 / 20$ \\
\hline $\begin{array}{l}\text { Abnormal LES relaxation } \\
>50 \% \text { of swallows }\end{array}$ & $18 / 19$ & $0 / 20$ \\
\hline $\begin{array}{l}\text { Abnormal LES relaxation } \\
\geq 20 \% \text { - }<50 \% \text { of swallows }\end{array}$ & $1 / 19$ & $0 / 20$ \\
\hline $\begin{array}{l}\text { Passage of ingesta from } \\
\text { esophagus to stomach } \\
\text { exclusively due to hydrostatic } \\
\text { pressure }\end{array}$ & $13 / 19$ & $0 / 20$ \\
\hline LES "Bird-Beak" & $12 / 19$ & $0 / 20$ \\
\hline Baseline Fluid Line & $13 / 19$ & $8 / 20$ \\
\hline Gastric Reflux & $1 / 19$ & $\mathrm{patints}$ \\
\hline
\end{tabular}

Table 10: Frequency of VFSS abnormalities in LES-AS patients compared to normal

controls. 
Table 11

\begin{tabular}{|c|c|c|c|c|}
\hline VFSS Parameter & Kappa & Standard Error & $95 \% \mathrm{CI}$ & Degree of Agreement \\
\hline $\begin{array}{l}\text { Presence of } \\
\text { functional LES } \\
\text { obstruction }\end{array}$ & 1.0 & 0.0 & $1-1$ & Perfect Agreement \\
\hline $\begin{array}{l}\text { Timing of ingesta } \\
\text { into the stomach } \\
\text { (with pharyngeal } \\
\text { swallow) }\end{array}$ & 0.7 & 0.2 & $0.5-1$ & Substantial Agreement \\
\hline $\begin{array}{l}\text { Timing of ingesta } \\
\text { into the stomach } \\
\text { (from hydrostatic } \\
\text { pressure) }\end{array}$ & 1.0 & 0.0 & $1-1$ & Perfect Agreement \\
\hline Baseline fluid line & 1.0 & 0.0 & $1-1$ & Perfect Agreement \\
\hline $\begin{array}{l}\text { Megaesophagus } \\
\text { (presence or absence } \\
\text { of ME) }\end{array}$ & 1.0 & 0.0 & $1-1$ & Perfect Agreement \\
\hline LES "Bird-beak" & 0.7 & 0.2 & $0.4-1$ & Substantial Agreement \\
\hline $\begin{array}{l}\text { Primary peristalsis } \\
\text { (contraction) }\end{array}$ & 0.8 & 0.3 & $0-0.9$ & Substantial Agreement \\
\hline $\begin{array}{l}\text { Primary peristalsis } \\
\text { (propulsion) }\end{array}$ & 0.5 & 0.6 & $0.2-0.8$ & Moderate Agreement \\
\hline $\begin{array}{l}\text { Hypermotile distal } \\
\text { esophagus }\end{array}$ & 0.7 & 0.2 & $0.3-1$ & Substantial Agreement \\
\hline $\begin{array}{l}\text { Consistency where } \\
\text { LES-AS was noted }\end{array}$ & 0.9 & 0.1 & $0.7-1$ & Near Perfect Agreement \\
\hline Secondary peristalsis & 0.8 & 0.1 & $0.6-1$ & Near Perfect Agreement \\
\hline Type of LES-AS & 0.8 & 0.1 & $0.5-1$ & Near Perfect Agreement \\
\hline $\begin{array}{l}\text { Reflux (abdominal } \\
\text { pressure) }\end{array}$ & 1.0 & 0.0 & $1-1$ & Perfect Agreement \\
\hline Reflux (spontaneous) & 0.9 & 0.1 & $0.8-1$ & Near Perfect Agreement \\
\hline Reflux (location) & 0.9 & 0.1 & $0.8-1$ & Near Perfect Agreement \\
\hline $\begin{array}{l}\text { Frequency of } \\
\text { observed LES } \\
\text { abnormalities }\end{array}$ & 0.3 & 0.3 & $0-0.9$ & Fair Agreement \\
\hline
\end{tabular}

Table 11. VFSS parameters with kappa/weighted kappa (linear weighting) coefficients,

standard error, 95\% CI, and degree of agreement between the MU panel and an

independent reviewer (CPG). 


\section{Table 12}

\begin{tabular}{|c|c|}
\hline Clinical Outcome Parameters & VFSS Outcome Parameters \\
\hline Overall client perception of clinical improvement & Presence of ME (yes or no) \\
\hline Body weight & $\begin{array}{l}\text { Esophageal motility and LES-AS subtype } \\
\text { - Type } 1 \\
\text { - Type } 2 \\
\text { - Type } 3 \\
\text { - } \quad \text { Normal motility }\end{array}$ \\
\hline Body condition score (9 point scale) & $\begin{array}{l}\text { Volume of gastric filling } \\
\text { - Sitting or standing (small), medium, } \\
\text { large) } \\
\text { - After } 5 \text { minutes upright (small, medium, } \\
\text { large) }\end{array}$ \\
\hline \multicolumn{2}{|l|}{ Frequency of regurgitation } \\
\hline \multicolumn{2}{|l|}{ Duration of clinical improvement } \\
\hline Complications & \\
\hline
\end{tabular}

Table 12: Clinical and VFSS outcome parameters for dogs having undergone mechanical

dilation + BTA injections for LES-AS. Patients were evaluated a median (IQR) of 21

days (14-25 days) post-treatment. 
Table 13

\begin{tabular}{|l|l|l|}
\hline & Pre-Treatment & Post-Treatment \\
\hline Presence of ME & $13 / 14$ & $13 / 14$ \\
\hline Esophageal motility & Type 1: 7/14 & Type 1: 7/14 \\
& Type 2: $5 / 14$ & Type 2: $5 / 14$ \\
& Type 3: $2 / 14$ & Type 3: $2 / 14$ \\
& Normal: $0 / 14$ & Normal: $0 / 14$ \\
\hline Volume of gastric filling (sitting & Small: $10 / 14$ & Small: 0/14 \\
or standing) & Medium: $4 / 14$ & Medium: $10 / 14$ \\
& Large: $0 / 14$ & Large: $4 / 14$ \\
\hline Volume of gastric filling (5 min & Small: $5 / 14$ & Small: $0 / 14$ \\
upright) & Medium: $7 / 14$ & Medium: $3 / 14$ \\
& Large: $2 / 14$ & Large: $11 / 14$ \\
\hline
\end{tabular}

Table 13: VFSS outcome parameters for dogs with LES-AS pre- and post-treatment with

LES mechanical dilation (pneumatic/bougienage) + BTA injections. For consistency,

dogs were evaluated in the same position (sitting or standing) in their pre-treatment and post-treatment evaluations. 
Table 14

\begin{tabular}{|l|l|}
\hline Region-of-Interest (ROI) & Median (IQR) \\
\hline P & $1.2 \%(0.6-1.6 \%)$ \\
\hline E3 & $0.8 \%(0.5-0.9 \%)$ \\
\hline E2 & $0.7 \%(0.6 \%-1.0 \%)$ \\
\hline E1 $*$ & $14.4 \%(7.7-27.4 \%)$ \\
\hline
\end{tabular}

Table 14: Median (IQR) reflux volume displayed for esophageal (E1-E3) and pharyngeal (P) regions of interest (ROI). Volume is displayed as a percent of gastric counts.(243) The volume of refluxate in the E1 ROI was significantly increased compared to E2, E3, and P ROI. E1: distal esophagus, E2: middle $3^{\text {rd }}$ of esophagus, E3: proximal $3^{\text {rd }}$ of the esophagus, * statistical significance 


\section{Table 15}

\begin{tabular}{|l|l|l|}
\hline \multicolumn{1}{|c|}{ Metabolic Protein } & \multicolumn{1}{c|}{ Tissue of Origin } & Relative Abundance \\
(Gastric Fluid)
\end{tabular}

Table 15: Metabolic proteins demonstrating increased abundance (GF vs. Normal) and

relative abundance in GF based on normalized spectral absorbance factors (NSAF) are

provided. Only proteins found within the 100 most abundant proteins are displayed

above. Gastric fluid (GF) 
Table 16

\begin{tabular}{|c|c|c|c|c|c|c|c|c|}
\hline \multirow{2}{*}{$\begin{array}{c}\text { Acoustic } \\
\text { Parameter } \\
\text { (units) }\end{array}$} & \multicolumn{8}{|c|}{ Acoustic Behavior } \\
\hline & Cough & Chew & Lick & Bark & Whine & Drink & Growl & $\begin{array}{l}\text { Throat- } \\
\text { clear }\end{array}$ \\
\hline Duration (sec) & $\begin{array}{l}2.1 \times 10^{-1} \\
(1.5- \\
2.0 \times 10^{-1)}\end{array}$ & $\begin{array}{l}1.7 \times 10^{-2} \\
(1.2- \\
\left.2.4 \times 10^{-2}\right)\end{array}$ & $\begin{array}{l}2.8 \times 10^{-2} \\
(2.0- \\
\left.5.2 \times 10^{-2}\right)\end{array}$ & $\begin{array}{l}4.0 \times 10^{\wedge-1} \\
(2.2- \\
\left.5.7 \times 10^{-1}\right)\end{array}$ & $\begin{array}{l}4.7 \times 10^{-1} \\
(2.5- \\
\left.5.8 \times 10^{-1}\right)\end{array}$ & $\begin{array}{l}5.0 \times 10^{-2} \\
(3.3- \\
\left.6.1 \times 10^{-2}\right)\end{array}$ & $\begin{array}{l}3.5 \times 10^{-1} \\
(2.8- \\
\left.5.3 \times 10^{-1}\right)\end{array}$ & $\begin{array}{l}1.5 \times 10^{\wedge-1} \\
(1.3- \\
\left..2 \times 10^{-1}\right)\end{array}$ \\
\hline $\begin{array}{l}\text { RMS amplitude } \\
\text { (unitless) }\end{array}$ & $\begin{array}{l}6.3 \times 10^{2} \\
(3.2- \\
\left.9.9 \times 10^{2}\right)\end{array}$ & $\begin{array}{l}4.7 \times 10^{2} \\
(2.3- \\
\left.9.0 \times 10^{2}\right)\end{array}$ & $\begin{array}{l}1.5 \times 10^{3}( \\
8.3 \times 10^{2}- \\
\left.2.3 \times 10^{3}\right)\end{array}$ & $\begin{array}{l}1.6 \times 10^{3} \\
(1.1- \\
\left.2 . \times 10^{3}\right)\end{array}$ & $\begin{array}{l}8.4 \times 10^{1} \\
\left(6.1 \times 10^{1}-\right. \\
\left.1.64 \times 10^{2}\right)\end{array}$ & $\begin{array}{l}1.8 \times 10^{2} \\
\left(6.5 \times 10^{1}-\right. \\
\left.2.3 \times 10^{2}\right)\end{array}$ & $\begin{array}{l}2.3 \times 10^{2} \\
(1.8- \\
\left.3.8 \times 10^{2}\right)\end{array}$ & $\begin{array}{l}1.9 \times 10^{2} \\
(1.2- \\
\left.5.5 \times 10^{2}\right)\end{array}$ \\
\hline $\begin{array}{l}\text { Maximum } \\
\text { amplitude } \\
\text { (unitless) }\end{array}$ & $\begin{array}{l}3.3 \times 10^{3} \\
(2.0- \\
\left.6.3 \times 10^{3}\right)\end{array}$ & $\begin{array}{l}1.7 \times 10^{3} \\
\left(9.1 \times 10^{2}\right. \\
- \\
\left.1.2 \times 10^{3}\right)\end{array}$ & $\begin{array}{l}4.7 \times 10^{3} \\
(3.7- \\
\left.6.5 \times 10^{3}\right)\end{array}$ & $\begin{array}{l}1.1 \times 10^{4} \\
\left(7.2 \times 10^{3}-\right. \\
\left.1.3 \times 10^{4}\right)\end{array}$ & $\begin{array}{l}6.2 \times 10^{2} \\
(1.2- \\
\left.3.5 \times 10^{2}\right)\end{array}$ & $\begin{array}{l}9.9 \times 10^{2} \\
\left(3.9 \times 10^{2}-\right. \\
\left.2.4 \times 10^{3}\right)\end{array}$ & $\begin{array}{l}8.8 \times 10^{2} \\
\left(6.1 \times 10^{2}-\right. \\
\left.1.2 \times 10^{3}\right)\end{array}$ & $\begin{array}{l}1.4 \times 10^{3} \\
\left(6.9 \times 10^{2}-\right. \\
\left.2.9 \times 10^{3}\right)\end{array}$ \\
\hline $\begin{array}{l}\text { Time to } \\
\text { maximum } \\
\text { amplitude (sec) }\end{array}$ & $\begin{array}{l}6.6 \times 10^{-2} \\
(5.5- \\
\left.7.6 \times 10^{-2}\right)\end{array}$ & $\begin{array}{l}9.0 \times 10^{-3} \\
\left(6.0 \times 10^{-}\right. \\
{ }^{3}-1.4 \times 10^{-} \\
\left.{ }^{-}\right)\end{array}$ & $\begin{array}{l}1.4 \times 10^{-2} \\
\left(9.0 \times 10^{-}\right. \\
{ }^{3}-6.3 \times 10^{-} \\
\left.{ }^{2}\right)\end{array}$ & $\begin{array}{l}1.2 \times 10^{-1} \\
\left(5.2 \times 10^{-2}-\right. \\
\left.1.7 \times 10^{-1}\right)\end{array}$ & $\begin{array}{l}3.2 \times 10^{-1} \\
(1.2- \\
\left.5.2 \times 10^{-1}\right)\end{array}$ & $\begin{array}{l}2.6 \times 10^{-2} \\
(1.8- \\
\left.3.5 \times 10^{-2}\right)\end{array}$ & $\begin{array}{l}1.5 \times 10^{-} \\
{ }^{1}\left(1.2-2 \times 10^{-}\right. \\
\left.{ }^{1}\right)\end{array}$ & $\begin{array}{l}4.9 \times 10^{-2} \\
(2.1- \\
\left.6.3 \times 10^{-2}\right)\end{array}$ \\
\hline $\begin{array}{l}\text { Slope } \\
\text { (unitless) }\end{array}$ & $\begin{array}{l}5.5 \times 10^{4} \\
(3.7- \\
\left.9.0 \times 10^{4}\right)\end{array}$ & $\begin{array}{l}1.9 \times 10^{5} \\
(1.1- \\
\left.4.1 \times 10^{5}\right)\end{array}$ & $\begin{array}{l}3.4 \times 10^{5} \\
(1.8- \\
\left.6.7 \times 10^{5}\right)\end{array}$ & $\begin{array}{l}1.1 \times 10^{5} \\
\left(6.8 \times 10^{4}-\right. \\
\left.2.2 \times 10^{5}\right)\end{array}$ & $\begin{array}{l}1.7 \times 10^{3} \\
\left(8.6 \times 10^{2}-\right. \\
\left.6.2 \times 10^{3}\right)\end{array}$ & $\begin{array}{l}1.4 \times 10^{4} \\
\left(7.1 \times 10^{3}-\right. \\
\left.4.8 \times 10^{4}\right)\end{array}$ & $\begin{array}{l}5.9 \times 10^{3} \\
(4.5- \\
\left.8.1 \times 10^{3}\right)\end{array}$ & $\begin{array}{l}2.8 \times 10^{4} \\
\left(2.2 \times 10^{4}-\right. \\
\left.1.3 \times 10^{5}\right)\end{array}$ \\
\hline $\begin{array}{l}\text { Inter-behavioral } \\
\text { interval } \\
(\mathrm{sec})\end{array}$ & $\begin{array}{l}1.3(1.0- \\
1.7)\end{array}$ & $\begin{array}{l}3.2 \times 10^{-1} \\
(2.8- \\
\left.3.5 \times 10^{-1}\right)\end{array}$ & $\begin{array}{l}2.6 \times 10^{-1} \\
(1.8- \\
\left.3.0 \times 10^{-1}\right)\end{array}$ & $\begin{array}{l}3.2 \times 10^{-1} \\
(1.4- \\
\left.9.6 \times 10^{-1}\right)\end{array}$ & $\begin{array}{l}2.4 \times 10^{-1} \\
(2.8- \\
\left.3.5 \times 10^{-1}\right)\end{array}$ & $\begin{array}{l}2.1 \times 10^{\wedge-1} \\
\left(6.6 \times 10^{-2}-\right. \\
\left.2.8 \times 10^{-1}\right)\end{array}$ & $\begin{array}{l}5.3 \times 10^{-1} \\
(2.2-3.6 \\
\left.\times 10^{-1}\right) \\
\end{array}$ & $\begin{array}{l}1.6(1.1- \\
1.9)\end{array}$ \\
\hline $\begin{array}{l}\text { Maximum } \\
\text { frequency }(\mathrm{Hz})\end{array}$ & $\begin{array}{l}1.7 \times 10^{2} \\
(1.7- \\
\left.3.5 \times 10^{2}\right)\end{array}$ & $\begin{array}{l}3.5 \times 10^{2} \\
\left(1.7 \times 10^{2}\right. \\
- \\
\left.1.0 \times 10^{3}\right)\end{array}$ & $\begin{array}{l}3.5 \times 10^{2} \\
(1.7- \\
\left.3.5 \times 10^{2}\right)\end{array}$ & $\begin{array}{l}6.9 \times 10^{2} \\
\left(5.2 \times 10^{\wedge 2}-\right. \\
\left.1.0 \times 10^{3}\right)\end{array}$ & $\begin{array}{l}5.2 \times 10^{2} \\
(5.2- \\
\left.7.8 \times 10^{2}\right)\end{array}$ & $\begin{array}{l}1.7 \times 10^{2} \\
(1.7- \\
\left.3.5 \times 10^{2}\right)\end{array}$ & $\begin{array}{l}1.7 \times 10^{2} \\
(1.7- \\
\left.5.6 \times 10^{2}\right)\end{array}$ & $\begin{array}{l}1.7 \times 10^{2} \\
(1.7- \\
\left.3.5 \times 10^{2}\right)\end{array}$ \\
\hline $\begin{array}{l}\text { Maximum } \\
\text { power } \\
\text { (dB) }\end{array}$ & $\begin{array}{l}8.7 \times 10^{1} \\
(8.2- \\
\left.9.0 \times 10^{1}\right)\end{array}$ & $\begin{array}{l}7.7(7.0- \\
\left.8.3 \times 10^{1}\right)\end{array}$ & $\begin{array}{l}9.1 \times 10^{1} \\
(8.6- \\
\left.9.4 \times 10^{1}\right)\end{array}$ & $\begin{array}{l}9.6 \times 10^{1}(9 . \\
\left.4-9.9 \times 10^{1}\right)\end{array}$ & $\begin{array}{l}7.2 \times 10^{1} \\
(6.7- \\
\left.7.8 \times 10^{1}\right)\end{array}$ & $\begin{array}{l}6.8 \times 10^{1} \\
(6.1- \\
\left.7.4 \times 10^{1}\right)\end{array}$ & $\begin{array}{l}7.7 \times 10^{1}(7 . \\
\left.4-7.9 \times 10^{1}\right)\end{array}$ & $\begin{array}{l}7.9 \times 10^{1} \\
(7.0- \\
\left.8.7 \times 10^{1}\right)\end{array}$ \\
\hline $\begin{array}{l}\text { Energy } \\
\text { (dB) }\end{array}$ & $\begin{array}{l}9.1 \times 10^{1} \\
(8.7- \\
\left.9.9 \times 10^{1}\right)\end{array}$ & $\begin{array}{l}8.2 \times 10^{1} \\
(7.5- \\
\left.8.7 \times 10^{1}\right)\end{array}$ & $\begin{array}{l}9.3 \times 10^{1} \\
(8.9- \\
\left.9.7 \times 10^{1}\right)\end{array}$ & $\begin{array}{l}1.1 \times 10^{2} \\
(1.0- \\
\left.1.1 \times 10^{2}\right)\end{array}$ & $\begin{array}{l}8.1 \times 10^{1} \\
(7.9- \\
\left.8.7 \times 10^{1}\right)\end{array}$ & $\begin{array}{l}7.2 \times 10^{1} \\
(6.7- \\
\left.7.8 \times 10^{1}\right)\end{array}$ & $\begin{array}{l}8.8 \times 10^{1} \\
(8.5- \\
\left.9.2 \times 10^{1}\right)\end{array}$ & $\begin{array}{l}8.3 \times 10^{1} \\
(7.9- \\
\left.9.1 \times 10^{1}\right)\end{array}$ \\
\hline
\end{tabular}

Table 16: Objective acoustic parameter (AP) data for each acoustic behavior (AB). Data

are presented as median (IQR). Definitions for each AP are provided in Table 1. Hertz

$(\mathrm{Hz})$, decibel (dB), seconds (sec). Amplitude measures are unitless.

Temporal Parameters: Duration: Time from start to the end of the AB; Root mean squared

(RMS) amplitude: Effective amplitude of the AB, Maximum amplitude: Maximum of

amplitude values in the AB, Time to maximum amplitude: Time to achieve maximum

amplitude, Slope: Maximum amplitude / Time, Inter-behavioral interval: Time between

$\mathrm{AB}$

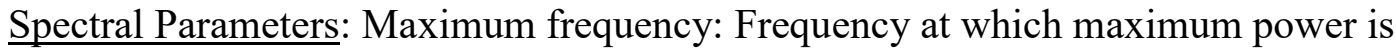


reached, Maximum power: Maximum power of the AB, Energy: Total energy within the bounds of the AB. 
Table 17

\begin{tabular}{|l|l|l|l|l|l|l|l|l|}
\hline & Cough & Chew & Lick & Bark & Whine & Drink & Growl & $\begin{array}{l}\text { Throat- } \\
\text { clear }\end{array}$ \\
\hline Cough & & 0.0001 & 0.0001 & 0.0001 & 0.0001 & 0.0002 & 0.0001 & 0.09 \\
\hline Chew & 0.0001 & & 0.0001 & 0.0001 & 0.0001 & 0.0001 & 0.0001 & 0.001 \\
\hline Lick & 0.0001 & 0.0001 & & 0.0001 & 0.0001 & 0.0001 & 0.0001 & 0.0001 \\
\hline Bark & 0.0001 & 0.0001 & 0.0001 & & 0.0001 & 0.0001 & 0.0001 & 0.0001 \\
\hline Whine & 0.001 & 0.0001 & 0.0001 & 0.0001 & & 0.0001 & 0.04 & 0.001 \\
\hline Drink & 0.0002 & 0.0001 & 0.0001 & 0.0001 & 0.0001 & & 0.0001 & 0.06 \\
\hline Growl & 0.0001 & 0.0001 & 0.0001 & 0.0001 & 0.04 & 0.0001 & & 0.001 \\
\hline $\begin{array}{l}\text { Throat- } \\
\text { clear }\end{array}$ & 0.09 & 0.001 & 0.0004 & 0.0001 & 0.001 & 0.06 & 0.001 & \\
\hline
\end{tabular}

Table 17: Analysis of similarity was performed for eight different $\mathrm{AB}$ with $P<0.001$

considered significant. Behavior comparisons reaching statistical significance suggest

that these behaviors are statistically dissimilar to each other. AB comparisons that did not reach statistical significance are highlighted in black. Cough was found to be dissimilar to all other $\mathrm{AB}$ with the exception of throat-clear. (R: 0.50) Acoustic behavior (AB) 
Table 18

\begin{tabular}{|l|l|l|}
\hline \multicolumn{1}{|c|}{ Acoustic Parameter } & \multicolumn{1}{c|}{$\begin{array}{c}\text { AB distinguished from } \\
\text { cough and TC }\end{array}$} & \multicolumn{1}{c|}{ P value } \\
\hline Duration (sec) & Chew, Lick, Drink & $<0.001$ \\
\hline RMS amplitude & Whine, Drink, Bark & $<0.001$ \\
\hline Maximum amplitude & Whine, Drink, Growl, Bark & $<0.001$ \\
\hline $\begin{array}{l}\text { Time to maximum amplitude } \\
\text { sec) }\end{array}$ & Chew, Lick, Drink & $<0.001$ \\
\hline Slope & Chew, Lick & $<0.001$ \\
\hline Inter-behavioral interval (sec) & Whine, Lick, Drink & $<0.001$ \\
\hline Maximum frequency (Hz) & Whine, Bark & $<0.001$ \\
\hline Maximum power (dB) & Whine Chew, Drink, Bark & $<0.001$ \\
\hline Energy (dB) & Whine, Chew, Drink, Bark & \\
\hline
\end{tabular}

Table 18: A one-way ANOVA on Ranks was performed to identify discriminatory acoustic parameters distinguishing respiratory from non-respiratory acoustic behaviors (AB). Respiratory AB, cough and throat-clear were distinguishable from all other AB by $\geq 2$ objective AP. $\mathrm{P}<0.05$ is considered significant. TC: Throat Clear. Hertz (Hz), decibel $(\mathrm{dB})$, seconds (sec) 
Table 19

\begin{tabular}{|c|c|c|c|c|c|c|c|c|}
\hline $\mathbf{A}$ & $\begin{array}{l}\text { Cough } \\
(\mathrm{n}=24)\end{array}$ & $\begin{array}{l}\text { Chew } \\
(n=30)\end{array}$ & $\begin{array}{l}\text { Lick } \\
(\mathrm{n}=30)\end{array}$ & $\begin{array}{l}\text { Bark } \\
(\mathrm{n}=30)\end{array}$ & $\begin{array}{l}\text { Whine } \\
(\mathrm{n}=16)\end{array}$ & $\begin{array}{l}\text { Drink } \\
(\mathrm{n}=30)\end{array}$ & $\begin{array}{l}\text { Growl } \\
(n=15)\end{array}$ & $\begin{array}{l}\text { Throat- } \\
\text { clear } \\
(\mathrm{n}=8)\end{array}$ \\
\hline $\begin{array}{l}\text { Correct } \\
\text { identification }\end{array}$ & 24 & 30 & 28 & 28 & 16 & 29 & 15 & 7 \\
\hline $\begin{array}{l}\text { Incorrect } \\
\text { identification }\end{array}$ & 0 & 0 & 2 & 2 & 0 & 1 & 0 & 1 \\
\hline $\begin{array}{l}\text { Percent correctly } \\
\text { identified }\end{array}$ & $100 \%$ & $100 \%$ & $93 \%$ & $93 \%$ & $100 \%$ & $97 \%$ & $100 \%$ & $89 \%$ \\
\hline B & \multicolumn{2}{|c|}{$\begin{array}{c}\text { Respiratory } \\
(\mathrm{n}=32)\end{array}$} & \multicolumn{3}{|c|}{ Vocalization $(\mathrm{n}=75)$} & \multicolumn{3}{|c|}{ Feeding $(\mathrm{n}=90)$} \\
\hline $\begin{array}{l}\text { Correct } \\
\text { identification }\end{array}$ & \multicolumn{2}{|c|}{32} & \multicolumn{3}{|l|}{73} & \multicolumn{3}{|l|}{90} \\
\hline $\begin{array}{l}\text { Incorrect } \\
\text { identification }\end{array}$ & \multicolumn{2}{|l|}{0} & \multicolumn{3}{|l|}{2} & \multicolumn{3}{|l|}{0} \\
\hline $\begin{array}{l}\text { Percent correctly } \\
\text { identified }\end{array}$ & \multicolumn{2}{|l|}{$100 \%$} & \multicolumn{3}{|l|}{$97 \%$} & \multicolumn{3}{|l|}{$100 \%$} \\
\hline
\end{tabular}

Table 19: A randomized sampling of waveforms were manually identified by a blinded

trained reviewer according to $\mathrm{AB}(\mathrm{A})$ with a total accuracy of $96.5 \%$. When grouped

according to category (i.e. respiratory, vocalization, and feeding behaviors) (B),

respiratory $\mathrm{AB}$ were correctly identified $100 \%$ of the time. Accuracy was calculated as

the percent of waveforms correctly identified. Strong reliability was calculated with a

kappa coefficient of 0.76 for individual $\mathrm{AB}$ assignment, and perfect agreement was

calculated for group assignment with a kappa of 0.97 . When categorized by group,

respiratory $\mathrm{AB}$ were correctly assigned $100 \%$ of the time. 


\section{BIBLIOGRAPHY}

1. Canning BJ, Chang AB, Bolser DC, Smith JA, Mazzone SB, McGarvey L. Anatomy and neurophysiology of cough: CHEST Guideline and Expert Panel report. Chest. 2014;146(6):1633-48.

2. Celli BR, Thomas NE, Anderson JA, Ferguson GT, Jenkins CR, Jones PW, et al. Effect of pharmacotherapy on rate of decline of lung function in chronic obstructive pulmonary disease: results from the TORCH study. American journal of respiratory and critical care medicine. 2008;178(4):332-8.

3. Gaude GS. Pulmonary manifestations of gastroesophageal reflux disease. Annals of Thoracic Medicine. 2009;4(3):115-23.

4. Yuksel ES, Vaezi MF. Extraesophageal manifestations of gastroesophageal reflux disease: cough, asthma, laryngitis, chest pain. Swiss Med Wkly. 2012;142:w13544.

5. Molyneux ID, Morice AH. Airway reflux, cough and respiratory disease. Therapeutic advances in chronic disease. 2011;2(4):237-48.

6. Irwin RS, Zawacki JK, Curley FJ, French CL, Hoffman PJ. Chronic cough as the sole presenting manifestation of gastroesophageal reflux. American Review of Respiratory Disease. 1989;140(5):1294-300.

7. Ramsey D, Smithard D, Kalra L. Silent aspiration: what do we know? Dysphagia. 2005;20(3):218-25.

8. Fass R, Dickman R. Clinical consequences of silent gastroesophageal reflux disease. Current Gastroenterology Reports. 2006;8(3):195-201.

9. Kahrilas PJ, Kia L. Pepsin: A Silent Biomarker for Reflux Aspiration or an Active Player in Extra-Esophageal Mucosal Injury? Chest. 2015;148(2):300-1.

10. Lu CL. Silent gastroesophageal reflux disease. Journal of neurogastroenterology and motility. 2012;18(3):236-8.

11. Dal Negro RW, Turco P, Micheletto C, Tognella S, Bonadiman L, Guerriero M, et al. Cost analysis of GER-induced asthma: a controlled study vs. atopic asthma of comparable severity. Respir Med. 2007;101(8):1814-20.

12. Nafe LAGMRC. Aspiration-related Respiratory Syndromes in the Dog JAVMA2016.

13. Praud JP. Upper airway reflexes in response to gastric reflux. Paediatric respiratory reviews. 2010;11(4):208-12.

14. Smith JA, Houghton LA. The oesophagus and cough: laryngo-pharyngeal reflux, microaspiration and vagal reflexes. Cough (London, England). 2013;9(1):12.

15. Loughlin CJ, Koufman JA, Averill DB, Cummins MM, Kim YJ, Little JP, et al. Acid-induced laryngospasm in a canine model. The Laryngoscope. 1996;106(12 Pt 1):1506-9. 
16. Takeuchi K, Nagahama K. Animal Model of Acid-Reflux Esophagitis: Pathogenic Roles of Acid/Pepsin, Prostaglandins, and Amino Acids. BioMed Research International. 2014;2014.

17. Poncet CM, Dupre GP, Freiche VG, Bouvy BM. Long-term results of upper respiratory syndrome surgery and gastrointestinal tract medical treatment in 51 brachycephalic dogs. The Journal of small animal practice. 2006;47(3):137-42.

18. Kempf J, Heinrich H, Reusch CE, Kook PH. Evaluation of esophageal highresolution manometry in awake and sedated dogs. American journal of veterinary research. 2013;74(6):895-900.

19. Kook P, Kempf J, Ruetten M, Reusch C. Wireless ambulatory esophageal pH monitoring in dogs with clinical signs interpreted as gastroesophageal reflux. Journal of veterinary internal medicine. 2014;28(6):1716-23.

20. Verschelden P, Cartier A, L'archeveque J, Trudeau C, Malo J. Compliance with and accuracy of daily self-assessment of peak expiratory flows (PEF) in asthmatic subjects over a three month period. European Respiratory Journal. 1996;9(5):880-5.

21. Logemann J. Swallowing physiology and pathophysiology. Otolaryngologic Clinics of North America. 1988;21(4):613-23.

22. Matsuo K, Palmer JB. Anatomy and physiology of feeding and swallowing: normal and abnormal. Physical medicine and rehabilitation clinics of North America. 2008;19(4):691-707, vii.

23. Bautista TG, Sun Q-J, Pilowsky PM. The generation of pharyngeal phase of swallow and its coordination with breathing: interaction between the swallow and respiratory central pattern generators. Progress in brain research. 212: Elsevier; 2014. p. 253-75.

24. Hollshwandner $\mathrm{CH}$, Brenman HS, Friedman MH. Role of afferent sensors in the initiation of swallowing in man. Journal of dental research. 1975;54(1):83-8.

25. Goyal RK, Mashimo H. Physiology of oral, pharyngeal, and esophageal motility. GI Motility online. 2006.

26. Neuhuber WL, Wörl J. Enteric co-innervation of striated muscle in the esophagus: still enigmatic? Histochemistry and Cell Biology. 2016;146(6):721-35.

27. Sandler AD, Schlegel JF, DeSautel MG, Maher JW. Neuroregulation of a chemosensitive afferent system in the canine distal esophagus. Journal of Surgical Research. 1993;55(4):364-71.

28. Lee AS, Ryu JH. Aspiration Pneumonia and Related Syndromes. Mayo Clinic proceedings. 2018;93(6):752-62.

29. Troche MS, Brandimore AE, Godoy J, Hegland KW. A framework for understanding shared substrates of airway protection. Journal of applied oral science : revista FOB. 2014;22(4):251-60. 
30. Ogura JH, Kawasaki M, Takenouchi S. LXXXVIII Neurophysiologic Observations on the Adaptive Mechanism of Deglutition. Annals of Otology, Rhinology \& Laryngology. 1964;73(4):1062-81.

31. Kawasaki M, H. Ogura J, Takenouchi S. Neurophysiologic observations of normal deglutition. I. Its relationship to the respiratory cycle2009. 1747-65 p.

32. McFarland DH, Lund J, Gagner M. Effects of posture on the coordination of respiration and swallowing. Journal of Neurophysiology. 1994;72(5):2431-7.

33. DeLegge MH. Aspiration pneumonia: incidence, mortality, and at - risk populations. Journal of parenteral and Enteral Nutrition. 2002;26:S19-S25.

34. Gross RD, Atwood Jr CW, Ross SB, Olszewski JW, Eichhorn KA. The coordination of breathing and swallowing in chronic obstructive pulmonary disease. American journal of respiratory and critical care medicine. 2009;179(7):559-65.

35. Oku Y, Dick TE. Phase resetting of the respiratory cycle before and after unilateral pontine lesion in cat. Journal of applied physiology (Bethesda, Md : 1985). 1992;72(2):721-30.

36. Gestreau C, Milano S, Bianchi AL, Grelot L. Activity of dorsal respiratory group inspiratory neurons during laryngeal-induced fictive coughing and swallowing in decerebrate cats. Experimental brain research. 1996;108(2):247-56.

37. Dick TE, Oku Y, Romaniuk JR, Cherniack NS. Interaction between central pattern generators for breathing and swallowing in the cat. The Journal of physiology. 1993;465:715-30.

38. Paydarfar D, Gilbert RJ, Poppel CS, Nassab PF. Respiratory phase resetting and airflow changes induced by swallowing in humans. The Journal of physiology. 1995;483 ( Pt 1):273-88.

39. Matsuo K, Palmer JB. Anatomy and physiology of feeding and swallowing: normal and abnormal. Physical medicine and rehabilitation clinics of North America. 2008;19(4):691-707.

40. Watrous BJ, Suter PF. Normal Swallowing in the Dog: A Cineradiographic Study. Veterinary Radiology. 1979;20(3-6):99-109.

41. Sanmiguel CP, Hagiike M, Mintchev MP, Cruz RD, Phillips EH, Cunneen SA, et al. Effect of electrical stimulation of the LES on LES pressure in a canine model. American Journal of Physiology-Gastrointestinal and Liver Physiology. 2008;295(2):G389-G94.

42. Pratschke KM, Bellenger CR, McAllister H, Campion D. Barrier pressure at the gastroesophageal junction in anesthetized dogs. American journal of veterinary research. 2001;62(7):1068-72.

43. Asoh R, Goyal RK. Manometry and electromyography of the upper esophageal sphincter in the opossum. Gastroenterology. 1978;74(3):514-20. 
44. Nishino T. Swallowing as a protective reflex for the upper respiratory tract. Anesthesiology. 1993;79(3):588-601.

45. Paterson WG, Hynna-Liepert TT, Selucky M. Comparison of primary and secondary esophageal peristalsis in humans: effect of atropine. The American journal of physiology. 1991;260(1 Pt 1):G52-7.

46. Paterson WG, Rattan S, Goyal RK. Experimental induction of isolated lower esophageal sphincter relaxation in anesthetized opossums. The Journal of clinical investigation. 1986;77(4):1187-93.

47. Nishino T, Takizawa K, Yokokawa N, Hiraga K. Depression of the swallowing reflex during sedation and/or relative analgesia produced by inhalation of $50 \%$ nitrous oxide in oxygen. Anesthesiology. 1987;67(6):995-8.

48. Shaker R, Ren J, Xie P, Lang IM, Bardan E, Sui Z. Characterization of the pharyngo-UES contractile reflex in humans. The American journal of physiology. 1997;273(4 Pt 1):G854-8.

49. Creamer B, Schlegel J. Motor responses of the esophagus to distention. Journal of applied physiology. 1957;10(3):498-504.

50. Aslam M, Kern M, Shaker R. Modulation of oesophago-UOS contractile reflex: effect of proximal and distal esophageal distention and swallowing.

Neurogastroenterology and motility : the official journal of the European Gastrointestinal Motility Society. 2003;15(3):323-9.

51. Gerhardt DC, Shuck TJ, Bordeaux RA, Winship DH. Human upper esophageal sphincter. Response to volume, osmotic, and acid stimuli. Gastroenterology. 1978;75(2):268-74.

52. Bajaj JS, Bajaj S, Dua KS, Jaradeh S, Rittmann T, Hofmann C, et al. Influence of sleep stages on esophago-upper esophageal sphincter contractile reflex and secondary esophageal peristalsis. Gastroenterology. 2006;130(1):17-25.

53. Babaei A, Bhargava V, Mittal RK. Upper esophageal sphincter during transient lower esophageal sphincter relaxation: effects of reflux content and posture. American journal of physiology Gastrointestinal and liver physiology. 2010;298(5):G601-7.

54. Medda BK, Lang IM, Layman R, Hogan WJ, Dodds WJ, Shaker R.

Characterization and quantification of a pharyngo-UES contractile reflex in cats. The American journal of physiology. 1994;267(6 Pt 1):G972-83.

55. Ren J, Shaker R, Medda B, Bonnevier J, Kern M, Dunn B, editors. Effect of acute esophagitis on the esophagoglottal closure reflex in a feline model. Gastroenterology; 1995: WB Saunders Co-Elsivier INC 1600 John kennedy Boulevard, STE 1800, Philadelphia, PE, 19103-2899 USA.

56. Kawamura O, Easterling C, Aslam M, Rittmann T, Hofmann C, Shaker R. Laryngo-upper esophageal sphincter contractile reflex in humans deteriorates with age. Gastroenterology. 2004;127(1):57-64. 
57. Shaker R, Dodds WJ, Ren J, Hogan WJ, Arndorfer RC. Esophagoglottal closure reflex: a mechanism of airway protection. Gastroenterology. 1992;102(3):857-61.

58. Shaker R, Ren J, Medda B, Lang I, Cowles V, Jaradeh S. Identification and characterization of the esophagoglottal closure reflex in a feline model. The American journal of physiology. 1994;266(1 Pt 1):G147-53.

59. Shaker R, Ren J, Hogan W, Liu J, Podvrsan B, Sui Z, editors. Glottal function during postprandial gastroesophageal reflux. Gastroenterology; 1993: WB Saunders Co Independence Square West Curtis Center, STE 300, Philadelphia

60. Lang IM, Medda BK, Shaker R. Mechanisms of reflexes induced by esophageal distension. American journal of physiology Gastrointestinal and liver physiology. 2001;281(5):G1246-63.

61. Ren J, Shaker R, Dua K, Trifan A, Podvrsan B, Sui Z, editors. Glottal adduction response to pharyngeal water stimulation-evidence for pharyngoglottal closure reflex Gastroenterology; 1994: WB Saunders CO Independence Square West Curtis Center, STE 300, Philidelphia

62. Shaker R, Medda BK, Ren J, Jaradeh S, Xie P, Lang IM. Pharyngoglottal closure reflex: identification and characterization in a feline model. American Journal of Physiology-Gastrointestinal and Liver Physiology. 1998;275(3):G521-G5.

63. Shaker R, Ren J, Bardan E, Easterling C, Dua K, Xie P, et al. Pharyngoglottal closure reflex: characterization in healthy young, elderly and dysphagic patients with predeglutitive aspiration. Gerontology. 2003;49(1):12-20.

64. Sasaki CT, Jassin B, Kim YH, Hundal J, Rosenblatt W, Ross DA. Central facilitation of the glottic closure reflex in humans. The Annals of otology, rhinology, and laryngology. 2003;112(4):293-7.

65. Chung KF, Pavord ID. Prevalence, pathogenesis, and causes of chronic cough. The Lancet. 2008;371(9621):1364-74.

66. Della Maggiore A. Tracheal and airway collapse in dogs. Veterinary Clinics: Small Animal Practice. 2014;44(1):117-27.

67. Canning BJ, Chang AB, Bolser DC, Smith JA, Mazzone SB, McGarvey L, et al. Anatomy and neurophysiology of cough: CHEST Guideline and Expert Panel report. Chest. 2014;146(6):1633-48.

68. Ross B, Gramiak R, Rahn H. Physical dynamics of the cough mechanism. Journal of applied physiology. 1955;8(3):264-8.

69. Troche MS, Brandimore AE, Godoy J, Hegland KW. A framework for understanding shared substrates of airway protection. Journal of Applied Oral Science. 2014;22(4):251-60.

70. Coleridge JC, Coleridge HM. Afferent vagal C fibre innervation of the lungs and airways and its functional significance. Reviews of Physiology, Biochemistry and Pharmacology, Volume 99: Springer; 1984. p. 1-110. 
71. Lee L-Y, Pisarri TE. Afferent properties and reflex functions of bronchopulmonary C-fibers. Respiration physiology. 2001;125(1-2):47-65.

72. Nassenstein C, Kwong K, Taylor - Clark T, Kollarik M, MacGlashan DM, Braun A, et al. Expression and function of the ion channel TRPA1 in vagal afferent nerves innervating mouse lungs. The Journal of physiology. 2008;586(6):1595-604.

73. Widdicombe J. Respiratory reflexes from the trachea and bronchi of the cat. The Journal of physiology. 1954;123(1):55-70.

74. Smith JA, Aliverti A, Quaranta M, McGuinness K, Kelsall A, Earis J, et al. Chest wall dynamics during voluntary and induced cough in healthy volunteers. The Journal of physiology. 2012;590(3):563-74.

75. Mazzone SB, Canning BJ. Central nervous system control of the airways: pharmacological implications. Current opinion in pharmacology. 2002;2(3):220-8.

76. Woolf CJ, Salter MW. Neuronal plasticity: increasing the gain in pain. science. 2000;288(5472):1765-8.

77. Salguero R, Herrtage M, Holmes M, Mannion P, Ladlow J. Comparison between computed tomographic characteristics of the middle ear in nonbrachycephalic and brachycephalic dogs with obstructive airway syndrome. Veterinary radiology \& ultrasound : the official journal of the American College of Veterinary Radiology and the International Veterinary Radiology Association. 2016;57(2):137-43.

78. Tarvin KM, Twedt DC, Monnet E. Prospective controlled study of gastroesophageal reflux in dogs with naturally occurring laryngeal paralysis. Veterinary surgery. 2016;45(7):916-21.

79. Lux CN, Archer TM, Lunsford KV. Gastroesophageal reflux and laryngeal dysfunction in a dog. Journal of the American Veterinary Medical Association. 2012;240(9):1100-3.

80. Turner RD, Bothamley GH. Chronic cough and a normal chest X-ray-a simple systematic approach to exclude common causes before referral to secondary care: a retrospective cohort study. NPJ primary care respiratory medicine. 2016;26:15081.

81. Mazzone SB, McGovern AE. Innervation of tracheal parasympathetic ganglia by esophageal cholinergic neurons: evidence from anatomic and functional studies in guinea pigs. American Journal of Physiology-Lung Cellular and Molecular Physiology. 2010;298(3):L404-L16.

82. Houghton LA, Lee AS, Badri H, DeVault KR, Smith JA. Respiratory disease and the oesophagus: reflux, reflexes and microaspiration. Nature reviews Gastroenterology \& hepatology. 2016.

83. Hu X, Yi ES, Ryu JH. Diffuse aspiration bronchiolitis: analysis of 20 consecutive patients. Jornal Brasileiro de Pneumologia. 2015;41:161-6.

84. Marik PE. Pulmonary aspiration syndromes. Current Opinion in Pulmonary Medicine. 2011;17(3):148-54. 
85. Gleeson K, Eggli DF, Maxwell SL. Quantitative aspiration during sleep in normal subjects. Chest. 1997;111(5):1266-72.

86. Hunt EB, Sullivan A, Galvin J, MacSharry J, Murphy DM. Gastric aspiration and Its role in airway inflammation. The Open Respiratory Medicine Journal. 2018;12:1.

87. Dent J, El-Serag H, Wallander MA, Johansson S. Epidemiology of gastrooesophageal reflux disease: a systematic review. Gut. 2005;54(5):710-7.

88. Talley NJ, Ford AC. Functional Dyspepsia. The New England journal of medicine. 2015;373(19):1853-63.

89. Raghavendran K, Nemzek J, Napolitano LM, Knight PR. Aspiration-induced lung injury. Critical care medicine. 2011;39(4):818-26.

90. Kennedy TP, Johnson KJ, Kunkel RG, Ward PA, Knight PR, Finch JS. Acute acid aspiration lung injury in the rat: biphasic pathogenesis. Anesthesia and analgesia. 1989;69(1):87-92.

91. Kennedy TP, Johnson KJ, Kunkel RG, Ward PA, Knight PR, Finch JS. Acute acid aspiration lung injury in the rat: biphasic pathogenesis. Anesthesia and analgesia. 1989;69(1):87-92.

92. Grimbert FA, Parker JC, Taylor AE. Increased pulmonary vascular permeability following acid aspiration. Journal of applied physiology: respiratory, environmental and exercise physiology. 1981;51(2):335-45.

93. Notter RH. Lung surfactants: basic science and clinical applications: CRC Press; 2000 .

94. Tobey NA, Hosseini SS, Caymaz-Bor C, Wyatt HR, Orlando GS, Orlando RC. The role of pepsin in acid injury to esophageal epithelium. The American journal of gastroenterology. 2001;96(11):3062.

95. Johnston N, Wells CW, Samuels TL, Blumin JH. Pepsin in nonacidic refluxate can damage hypopharyngeal epithelial cells. Annals of Otology, Rhinology \& Laryngology. 2009;118(9):677-85.

96. Gill GA, Johnston N, Buda A, Pignatelli M, Pearson J, Dettmar PW, et al. Laryngeal epithelial defenses against laryngopharyngeal reflux: investigations of Ecadherin, carbonic anhydrase isoenzyme III, and pepsin. Annals of Otology, Rhinology \& Laryngology. 2005;114(12):913-21.

97. Raghavendran K, Nemzek J, Napolitano LM, Knight PR. Aspiration-Induced lung injury. Critical care medicine. 2011;39(4):818-26.

98. Määttä OM, Laurila HP, Holopainen S, Lilja - Maula L, Melamies M, Viitanen $\mathrm{SJ}$, et al. Reflux aspiration in lungs of dogs with respiratory disease and in healthy West Highland White Terriers. Journal of veterinary internal medicine. 2018;32(6):2074-81. 
99. Pauwels A, Decraene A, Blondeau K, Mertens V, Farre R, Proesmans M, et al. Bile acids in sputum and increased airway inflammation in patients with cystic fibrosis. Chest. 2012;141.

100. Grabowski M, Kasran A, Seys S, Pauwels A, Medrala W, Dupont L, et al. Pepsin and bile acids in induced sputum of chronic cough patients. Respir Med. 2011;105.

101. Ovbey DH, Wilson DV, Bednarski RM, Hauptman JG, Stanley BJ, Radlinsky

MG, et al. Prevalence and risk factors for canine post - anesthetic aspiration pneumonia (1999-2009): a multicenter study. Veterinary anaesthesia and analgesia. 2014;41(2):12736.

102. Kogan DA, Johnson LR, Sturges BK, Jandrey KE, Pollard RE. Etiology and clinical outcome in dogs with aspiration pneumonia: 88 cases (2004-2006). Journal of the American Veterinary Medical Association. 2008;233(11):1748-55.

103. Dal Negro RW, Turco P, Micheletto C, Tognella S, Bonadiman L, Guerriero M, et al. Cost analysis of GER-induced asthma: A controlled study vs. atopic asthma of comparable severity. Respiratory medicine. 2007;101(8):1814-20.

104. Hawkins EC, Basseches J, Berry CR, Stebbins ME, Ferris KK. Demographic, clinical, and radiographic features of bronchiectasis in dogs: 316 cases (1988-2000). Journal of the American Veterinary Medical Association. 2003;223(11):1628-35.

105. Johnson LR. Laryngeal structure and function in dogs with cough. Journal of the American Veterinary Medical Association. 2016;249(2):195-201.

106. Stanley BJ, Hauptman JG, Fritz MC, Rosenstein DS, Kinns J. Esophageal dysfunction in dogs with idiopathic laryngeal paralysis: a controlled cohort study. Veterinary surgery : VS. 2010;39(2):139-49.

107. Irwin RS. Chronic cough due to gastroesophageal reflux disease: ACCP evidencebased clinical practice guidelines. Chest. 2006;129(1 Suppl):80s-94s.

108. Palombini BC, Villanova CAC, Araújo E, Gastal OL, Alt DC, Stolz DP, et al. A Pathogenic Triad in Chronic Cough: Asthma, Postnasal Drip Syndrome, and Gastroesophageal Reflux Disease. Chest. 1999;116(2):279-84.

109. Johnson EG, Wisner ER. Advances in Respiratory Imaging. Veterinary Clinics of North America: Small Animal Practice. 2007;37(5):879-900.

110. Wielpütz MO, Heußel CP, Herth FJ, Kauczor HU. Radiological diagnosis in lung disease: factoring treatment options into the choice of diagnostic modality. Deutsches Arzteblatt international. 2014;111(11):181-7.

111. Mantis P, Lamb C, Boswood A. Assessment of the accuracy of thoracic radiography in the diagnosis of canine chronic bronchitis. Journal of small animal practice. 1998;39(11):518-20.

112. Postma GN, Belafsky PC, Aviv JE, Koufman JA. Laryngopharyngeal reflux testing. Ear, nose, \& throat journal. 2002;81(9 Suppl 2):14-8. 
113. Vaezi MF. New Tests for the Evaluation of Laryngopharyngeal Reflux. Gastroenterology \& Hepatology. 2013;9(2):115-7.

114. Pollard RE. Imaging Evaluation of Dogs and Cats with Dysphagia. ISRN Vet Sci. 2012;2012.

115. Potluri S, Friedenberg F, Parkman HP, Chang A, MacNeal R, Manus C, et al. Comparison of a salivary/sputum pepsin assay with 24-hour esophageal $\mathrm{pH}$ monitoring for detection of gastric reflux into the proximal esophagus, oropharynx, and lung. Digestive diseases and sciences. 2003;48(9):1813-7.

116. Vaezi MF. Diagnosing Gastroesophageal Reflux Disease With EndoscopicGuided Mucosal Impedance. Gastroenterology \& Hepatology. 2016;12(4):266.

117. Sidhwa F, Moore A, Alligood E, Fisichella PM. Diagnosis and Treatment of the Extraesophageal Manifestations of Gastroesophageal Reflux Disease. LWW; 2016.

118. Wenzl TG, Moroder C, Trachterna M, Thomson M, Silny J, Heimann G, et al. Esophageal $\mathrm{pH}$ monitoring and impedance measurement: a comparison of two diagnostic tests for gastroesophageal reflux. Journal of pediatric gastroenterology and nutrition. 2002;34(5):519-23.

119. Kook PH, Kempf J, Ruetten M, Reusch CE. Wireless ambulatory esophageal pH monitoring in dogs with clinical signs interpreted as gastroesophageal reflux. Journal of veterinary internal medicine. 2014;28(6):1716-23.

120. Shaheen NJ, Crockett SD, Bright SD, Madanick RD, Buckmire R, Couch M, et al. Randomised clinical trial: high - dose acid suppression for chronic cough-a double blind, placebo - controlled study. Alimentary pharmacology \& therapeutics. 2011;33(2):225-34.

121. Faruqi S, Molyneux ID, Fathi H, Wright C, Thompson R, Morice AH. Chronic cough and esomeprazole: a double-blind placebo-controlled parallel study. Respirology. 2011;16(7):1150-6.

122. Bardhan KD, Strugala V, Dettmar PW. Reflux revisited: advancing the role of pepsin. International journal of otolaryngology. 2012;2012:646901.

123. Johnston N, Dettmar PW, Bishwokarma B, Lively MO, Koufman JA. Activity/stability of human pepsin: implications for reflux attributed laryngeal disease. The Laryngoscope. 2007;117(6):1036-9.

124. Sifrim D, Holloway R, Silny J, Xin Z, Tack J, Lerut A, et al. Acid, nonacid, and gas reflux in patients with gastroesophageal reflux disease during ambulatory 24-hour pH-impedance recordings. Gastroenterology. 2001;120(7):1588-98.

125. Harris R, Grobman M, Allen M, Schachtel J, Rawson N, Bennett B, et al. Standardization of a Videofluoroscopic Swallow Study Protocol to Investigate Dysphagia in Dogs. Journal of veterinary internal medicine. 2017;31(2):383-93.

126. Neil JA, Canapp Jr SO, Cook CR, Lattimer JC. Kartagener's syndrome in a dachshund dog. Journal of the American Animal Hospital Association. 2002;38(1):45-9. 
127. Elbl B, Birkenfeld B, Walecka A, Szymanowicz J, Listewnik M, Gwardyś A, et al. Upper gastrointestinal tract scintigraphy and ultrasonography in diagnosis of gastroesophageal reflux in children. Polish Journal of Radiology. 2011;76(1):63.

128. Ravelli AM, Panarotto MB, Verdoni L, Consolati V, Bolognini S. Pulmonary aspiration shown by scintigraphy in gastroesophageal reflux-related respiratory disease. CHEST Journal. 2006;130(5):1520-6.

129. Silver KH, Van Nostrand D. The use of scintigraphy in the management of patients with pulmonary aspiration. Dysphagia. 1994;9(2):107-15.

130. Falk GL, Beattie J, Ing A, Falk S, Magee M, Burton L, et al. Scintigraphy in laryngopharyngeal and gastroesophageal reflux disease: a definitive diagnostic test? World Journal of Gastroenterology: WJG. 2015;21(12):3619.

131. Songür N, Songür Y, Cerci SS, Öztürk Ö, Sahin Ü, Senol A, et al.

Gastroesophageal scintigraphy in the evaluation of adult patients with chronic cough due to gastroesophageal reflux disease. Nuclear medicine communications.

2008;29(12):1066-72.

132. Ceciliani F, Eckersall D, Burchmore R, Lecchi C. Proteomics in veterinary medicine: applications and trends in disease pathogenesis and diagnostics. Veterinary pathology. 2014;51(2):351-62.

133. Samuels TL, Johnston N. Pepsin as a marker of extraesophageal reflux. The Annals of otology, rhinology, and laryngology. 2010;119(3):203-8.

134. Amoh J, Odame K. Technologies for developing ambulatory cough monitoring devices. Critical reviews in biomedical engineering. 2013;41(6):457-68.

135. Abeyratne UR, Swarnkar V, Setyati A, Triasih R. Cough sound analysis can rapidly diagnose childhood pneumonia. Annals of biomedical engineering. 2013;41(11):2448-62.

136. Chen L, Lai K, Lomask JM, Jiang B, Zhong N. Detection of mouse cough based on sound monitoring and respiratory airflow waveforms. PloS one. 2013;8(3):e59263.

137. Ferrari S, Silva M, Guarino M, Aerts JM, Berckmans D. Cough sound analysis to identify respiratory infection in pigs. Computers and electronics in agriculture.

2008;64(2):318-25.

138. Korpas J, Sadlonova J, Salat D, Masarova E. The origin of cough sounds. Bulletin europeen de physiopathologie respiratoire. 1986;23:47s-50s.

139. Korpas J, Sadlonova J, Vrabec M. Analysis of the cough sound: an overview. Pulmonary pharmacology. 1996;9(5-6):261-8.

140. Coca-Pelaz A, Rodrigo JP, Paccagnella D, Takes RP, Rinaldo A, Silver CE, et al. Reflux and aerodigestive tract diseases. European Archives of Oto-Rhino-Laryngology. 2013;270(2):417-23. 
141. de Benedictis FM, Bush A. Respiratory manifestations of gastro-oesophageal reflux in children. Archives of disease in childhood. 2018;103(3):292-6.

142. Burke JM, Jackson W, Morice AH. The role of high resolution oesophageal manometry in occult respiratory symptoms. Respiratory medicine. 2018;138:47-9.

143. Houghton LA, Lee AS, Badri H, DeVault KR, Smith JA. Respiratory disease and the oesophagus: reflux, reflexes and microaspiration. Nature reviews Gastroenterology \& hepatology. 2016;13(8):445-60.

144. Nafe LA, Grobman ME, Masseau I, Reinero CR. Aspiration-related respiratory disorders in dogs. Journal of the American Veterinary Medical Association. 2018;253(3):292-300.

145. Turner RD, Bothamley GH. Chronic cough and a normal chest X-ray - a simple systematic approach to exclude common causes before referral to secondary care: a retrospective cohort study. NPJ primary care respiratory medicine. 2016;26:15081.

146. Achilleos A. Evidence-based Evaluation and Management of Chronic Cough. The Medical clinics of North America. 2016;100(5):1033-45.

147. Harris RA, Grobman ME, Allen MJ, Schachtel J, Rawson NE, Bennett B, et al. Standardization of a Videofluoroscopic Swallow Study Protocol to Investigate Dysphagia in Dogs. Journal of veterinary internal medicine. 2017;31(2):383-93.

148. Grobman M. Videofluoroscopic swallow study features of obstructive lower esophageal sphincter (LES) disorders in dogs ACVIM Forum 2017.

149. Tarvin KM, Twedt DC, Monnet E. Prospective Controlled Study of Gastroesophageal Reflux in Dogs with Naturally Occurring Laryngeal Paralysis. Veterinary surgery : VS. 2016;45(7):916-21.

150. Skerrett SC, McClaran JK, Fox PR, Palma D. Clinical Features and Outcome of Dogs with Epiglottic Retroversion With or Without Surgical Treatment: 24 Cases. Journal of veterinary internal medicine. 2015;29(6):1611-8.

151. Bottero E, Bellino C, De Lorenzi D, Ruggiero P, Tarducci A, D'Angelo A, et al. Clinical evaluation and endoscopic classification of bronchomalacia in dogs. Journal of veterinary internal medicine. 2013;27(4):840-6.

152. Johnson LR, Johnson EG, Vernau W, Kass PH, Byrne BA. Bronchoscopy, Imaging, and Concurrent Diseases in Dogs with Bronchiectasis: (2003-2014). Journal of veterinary internal medicine. 2016;30(1):247-54.

153. Meola SD. Brachycephalic airway syndrome. Topics in companion animal medicine. 2013;28(3):91-6.

154. King LG. Textbook of respiratory disease in dogs and cats: WB Saunders; 2004.

155. Grobman M, Reinero C. Investigation of Neurokinin-1 Receptor Antagonism as a Novel Treatment for Chronic Bronchitis in Dogs. Journal of veterinary internal medicine. 2016;30(3):847-52. 
156. Martin BJ, Corlew MM, Wood H, Olson D, Golopol LA, Wingo M, et al. The association of swallowing dysfunction and aspiration pneumonia. Dysphagia.

1994;9(1):1-6.

157. Pollard RE, Marks SL, Cheney DM, Bonadio CM. Diagnostic outcome of contrast videofluoroscopic swallowing studies in 216 dysphagic dogs. Veterinary radiology \& ultrasound : the official journal of the American College of Veterinary Radiology and the International Veterinary Radiology Association. 2017;58(4):373-80.

158. Pollard RE. Imaging evaluation of dogs and cats with Dysphagia. ISRN veterinary science. 2012;2012:238505.

159. Decalmer S, Stovold R, Houghton LA, Pearson J, Ward C, Kelsall A, et al. Chronic cough: relationship between microaspiration, gastroesophageal reflux, and cough frequency. Chest. 2012;142(4):958-64.

160. Wilson D, Monnet E. Risk factors for the development of aspiration pneumonia after unilateral arytenoid lateralization in dogs with laryngeal paralysis: 232 cases (19872012). Journal of the American Veterinary Medical Association. 2016;248(2):188-94.

161. Smith JA, Decalmer S, Kelsall A, McGuinness K, Jones H, Galloway S, et al. Acoustic Cough-Reflux Associations in Chronic Cough: Potential Triggers and Mechanisms. Gastroenterology. 2010;139(3):754-62.

162. Sontag SJ. The spectrum of pulmonary symptoms due to gastroesophageal reflux. Thoracic Surgery Clinics. 2005;15(3):353-68.

163. Ozdemir P, Erdinc M, Vardar R, Veral A, Akyildiz S, Ozdemir O, et al. The Role of Microaspiration in the Pathogenesis of Gastroesophageal Reflux-related Chronic Cough. Journal of neurogastroenterology and motility. 2017;23(1):41-8.

164. Madanick RD. Extraesophageal presentations of GERD: where is the science? Gastroenterology clinics of North America. 2014;43(1):105-20.

165. Stanley BJ, Hauptman JG, Fritz MC, Rosenstein DS, Kinns J. Esophageal dysfunction in dogs with idiopathic laryngeal paralysis: a controlled cohort study. Veterinary surgery. 2010;39(2):139-49.

166. Woodcock A, Young EC, Smith JA. New insights in cough. British medical bulletin. 2010;96:61-73.

167. Tibbling L. Wrong-way swallowing as a possible cause of bronchitis in patients with gastroesophageal reflux disease. Acta oto-laryngologica. 1993;113(3):405-8.

168. Dorgan DJ, Tino G, O’Donnell A. Diagnostic approach to bronchiectasis. Current Pulmonology Reports. 2015;4(4):191-7.

169. Boogaard R, Huijsmans SH, Pijnenburg MWH, Tiddens HAWM, de Jongste JC, Merkus PJFM. Tracheomalacia and Bronchomalacia in Children: Incidence and Patient Characteristics. Chest. 2005;128(5):3391-7. 
170. Bibi H, Khvolis E, Shoseyov D, Ohaly M, Ben Dor D, London D, et al. The prevalence of gastroesophageal reflux in children with tracheomalacia and laryngomalacia. Chest. 2001;119(2):409-13.

171. Friedman B, Frazier JB. Deep Laryngeal Penetration as a Predictor of Aspiration. Dysphagia. 2000;15(3):153-8.

172. McBrearty AR, Ramsey IK, Courcier EA, Mellor DJ, Bell R. Clinical factors associated with death before discharge and overall survival time in dogs with generalized megaesophagus. Journal of the American Veterinary Medical Association.

2011;238(12):1622-8.

173. Mace S, Shelton G, Eddlestone S. Megaesophagus. Compendium (Yardley, PA). 2012;34(2):E1-E.

174. Harvey C, O'Brien J, Durie V, Miller D, Veenema R. Megaesophagus in the dog: a clinical survey of 79 cases. Journal of the American Veterinary Medical Association. 1974;165(5):443-6.

175. Mears EA, Jenkins CC. Canine and feline megaesophagus. Compendium on Continuing Education for the Practicing Veterinarian. 1997;19(3):313-26.

176. Shelton GD. Myasthenia gravis and congenital myasthenic syndromes in dogs and cats: a history and mini-review. Neuromuscular Disorders. 2016;26(6):331-4.

177. Gaynor A, Shofer F, Washabau R. Risk factors for acquired megaesophagus in dogs. Journal of the American Veterinary Medical Association. 1997;211(11):1406-12.

178. Gyawali C. Achalasia: new perspectives on an old disease. Neurogastroenterology \& Motility. 2016;28(1):4-11.

179. Kahrilas PJ, Boeckxstaens G. The spectrum of achalasia: lessons from studies of pathophysiology and high-resolution manometry. Gastroenterology. 2013;145(5):954-65.

180. Pohl D, Tutuian R. Achalasia: an overview of diagnosis and treatment. Journal of Gastrointestinal and liver diseases. 2007;16(3):297.

181. Furuzawa-Carballeda J, Torres-Landa S, Valdovinos M, Coss-Adame E, Martín Del Campo LA, Torres-Villalobos G. New insights into the pathophysiology of achalasia and implications for future treatment. World journal of gastroenterology. 2016;22(35):7892-907.

182. Kempf J, Beckmann K, Kook P. Achalasia - Like Disease with Esophageal Pressurization in a Myasthenic Dog. Journal of veterinary internal medicine. 2014;28(2):661-5.

183. Osborne C, Clifford D, Jessen C. Hereditary esophageal achalasia in dogs. Journal of the American Veterinary Medical Association. 1967;151(5):572-81.

184. Palmer C. Achalasia or cardiospasm in Great Dane puppies. Veterinary medicine, small animal clinician: VM, SAC. 1968;63(6):574-6. 
185. Quintavalla F, Menozzi A, Pozzoli C, Poli E, Donati P, Wyler D, et al. Sildenafil improves clinical signs and radiographic features in dogs with congenital idiopathic megaoesophagus: a randomised controlled trial. Veterinary Record. 2017;180(16):404-.

186. Kahrilas PJ, Bredenoord AJ, Fox M, Gyawali CP, Roman S, Smout A, et al. Advances in the management of oesophageal motility disorders in the era of highresolution manometry: a focus on achalasia syndromes. Nature reviews Gastroenterology \& hepatology. 2018;15(5):323.

187. Boeckxstaens G, Zaninotto G. Achalasia and esophago - gastric junction outflow obstruction: focus on the subtypes. Neurogastroenterology \& Motility. 2012;24(s1):2731 .

188. Neyaz Z, Gupta M, Ghoshal UC. How to perform and interpret timed barium esophagogram. Journal of neurogastroenterology and motility. 2013;19(2):251.

189. Jung DH, Park H. Is Gastroesophageal Reflux Disease and Achalasia Coincident or Not? Journal of neurogastroenterology and motility. 2017;23(1):5-8.

190. Gyawali C, Bredenoord A, Conklin J, Fox M, Pandolfino J, Peters J, et al. Evaluation of esophageal motor function in clinical practice. Neurogastroenterology \& Motility. 2013;25(2):99-133.

191. El-Takli I, O'Brien P, Paterson W. Clinical diagnosis of achalasia: how reliable is the barium x-ray? Canadian Journal of Gastroenterology and Hepatology. 2006;20(5):335-7.

192. Kahrilas PJ, Pandolfino JE. Treatments for achalasia in 2017: how to choose among them. Current opinion in gastroenterology. 2017;33(4):270-6.

193. Ullal TV, Kass PH, Conklin JL, Belafsky PC, Marks SL. High-resolution manometric evaluation of the effects of cisapride on the esophagus during administration of solid and liquid boluses in awake healthy dogs. American journal of veterinary research. 2016;77(8):818-27.

194. Kempf J, Lewis F, Reusch CE, Kook PH. High-resolution manometric evaluation of the effects of cisapride and metoclopramide hydrochloride administered orally on lower esophageal sphincter pressure in awake dogs. American journal of veterinary research. 2014;75(4):361-6.

195. Pollard RE. Imaging evaluation of dogs and cats with dysphagia. ISRN Vet Sci. $2012 ; 2012$.

196. Pollard RE, Marks SL, Cheney DM, Bonadio CM. Diagnostic outcome of contrast videofluoroscopic swallowing studies in 216 dysphagic dogs. Veterinary Radiology \& Ultrasound. 2017;58(4):373-80.

197. Martinez C, Targarona EM, Sainz S, Cerdan G, Novell J, Trias M. [Pseudoachalasia: a diagnosis to consider in the assessment of dysphagia]. Gastroenterologia y hepatologia. 2000;23(1):14-5. 
198. Viera AJ, Garrett JM. Understanding interobserver agreement: the kappa statistic. Family medicine. 2005;37(5):360-3.

199. Gyawali CP, Bredenoord AJ, Conklin JL, Fox M, Pandolfino JE, Peters JH, et al. Evaluation of esophageal motor function in clinical practice. Neurogastroenterology and motility : the official journal of the European Gastrointestinal Motility Society. 2013;25(2):99-133.

200. Pasricha PJ, Rai R, Ravich WJ, Hendrix TR, Kalloo AN. Botulinum toxin for achalasia: long-term outcome and predictors of response. Gastroenterology. 1996;110(5):1410-5.

201. Vaezi MF, Felix VN, Penagini R, Mauro A, de Moura EG, Pu LZ, et al. Achalasia: from diagnosis to management. Ann N Y Acad Sci. 2016;29(10):13176.

202. Eckardt V, Gockel I, Bernhard G. Pneumatic dilation for achalasia: late results of a prospective follow up investigation. Gut. 2004;53(5):629-33.

203. Huh CW, Youn YH, Chung H, Lee YC, Park H. Functional restoration of the esophagus after peroral endoscopic myotomy for achalasia. PloS one.

2017;12(5):e0178414.

204. Vaezi MF, Pandolfino JE, Vela MF. ACG Clinical Guideline: Diagnosis and Management of Achalasia. The American Journal Of Gastroenterology. 2013;108:1238.

205. Dobrucali A, Erzin Y, Tuncer M, Dirican A. Long-term results of graded pneumatic dilatation under endoscopic guidance in patients with primary esophageal achalasia. World journal of gastroenterology. 2004;10(22):3322-7.

206. Bonavina L, Nosadini A, Bardini R, Baessato M, Peracchia A. Primary treatment of esophageal achalasia: long-term results of myotomy and Dor fundoplication. Archives of surgery. 1992;127(2):222-7.

207. Knecht C, Eaddy J. Canine esophageal achalasia corrected by retrograde dilatation--a case report. Journal of the American Veterinary Medical Association. 1959;135:554.

208. McBrearty AR, Ramsey IK, Courcier EA, Mellor DJ, Bell R. Clinical factors associated with death before discharge and overall survival time in dogs with generalized megaesophagus. J Am Vet Med Assoc. 2011;238(12):1622-8.

209. Palmer CS. Achalasia or cardiospasm in Great Dane puppies. Vet Med Small Anim Clin. 1968;63(6):574-6.

210. Poppel MH, Lust FJ. Achalasia of the esophagus in a dachshund. Am J Roentgenol Radium Ther Nucl Med. 1962;88:741-2.

211. Stack W, Thomson J, Suyama A. Achalasia of the esophagus with megaesophagus in a dog. Journal of the American Veterinary Medical Association. 1957;131(5):225-6. 
212. Boria PA, Webster CR, Berg J. Esophageal achalasia and secondary megaesophagus in a dog. The Canadian Veterinary Journal. 2003;44(3):232.

213. Earlam RJ, Zollman PE, Ellis FH. Congenital oesophageal achalasia in the dog. Thorax. 1967;22(5):466-72.

214. El-Takli I, O'Brien P, Paterson WG. Clinical diagnosis of achalasia: how reliable is the barium $\mathrm{x}$-ray? Canadian journal of gastroenterology $=$ Journal canadien de gastroenterologie. 2006;20(5):335-7.

215. Diamant N, Szczepanski M, Mui H. Manometric characteristics of idiopathic megaesophagus in the dog: an unsuitable animal model for achalasia in man. Gastroenterology. 1973;65(2):216-23.

216. Bortolotti M, Mari C, Lopilato C, Porrazzo G, Miglioli M. Effects of sildenafil on esophageal motility of patients with idiopathic achalasia. Gastroenterology. 2000;118(2):253-7.

217. Uppal DS, Wang AY. Update on the endoscopic treatments for achalasia. World journal of gastroenterology. 2016;22(39):8670-83.

218. Moonen A, Boeckxstaens G. Finding the right treatment for achalasia treatment: risks, efficacy, complications. Current treatment options in gastroenterology. 2016;14(4):420-8.

219. Yeates J, Main D. Assessment of companion animal quality of life in veterinary practice and research. Journal of Small Animal Practice. 2009;50(6):274-81.

220. Vaezi MF, Baker ME, Achkar E, Richter JE. Timed barium oesophagram: better predictor of long term success after pneumatic dilation in achalasia than symptom assessment. Gut. 2002;50(6):765-70.

221. Roman S, Kahrilas PJ, Mion F, Nealis TB, Soper NJ, Poncet G, et al. Partial recovery of peristalsis after myotomy for achalasia: more the rule than the exception. JAMA surgery. 2013;148(2):157-64.

222. Patti M, Galvani C, Gorodner M, Tedesco P. Timing of surgical intervention does not influence return of esophageal peristalsis or outcome for patients with achalasia. Surgical Endoscopy And Other Interventional Techniques. 2005;19(9):1188-92.

223. Horgan S, Hudda K, Eubanks T, McAllister J, Pellegrini C. Does botulinum toxin injection make esophagomyotomy a more difficult operation? Surgical endoscopy. 1999;13(6):576-9.

224. Patti MG, Feo CV, Arcerito M, De Pinto M, Tamburini A, Diener U, et al. Effects of previous treatment on results of laparoscopic Heller myotomy for achalasia. Digestive diseases and sciences. 1999;44(11):2270-6.

225. Yamaguchi D, Tsuruoka N, Sakata Y, Shimoda R, Fujimoto K, Iwakiri R. Safety and efficacy of botulinum toxin injection therapy for esophageal achalasia in Japan. Journal of Clinical Biochemistry and Nutrition. 2015;57(3):239-43. 
226. Eckardt AJ, Eckardt VF. Current clinical approach to achalasia. World journal of gastroenterology. 2009;15(32):3969-75.

227. Annese V, Bassotti G, Coccia G, Dinelli M, D'Onofrio V, Gatto G, et al. A multicentre randomised study of intrasphincteric botulinum toxin in patients with oesophageal achalasia. Gut. 2000;46(5):597-600.

228. Kuhn MA, Belafsky PC. Management of Cricopharyngeus Muscle Dysfunction. Otolaryngologic Clinics of North America. 2013;46(6):1087-99.

229. Kozarek R, Gelfand M, Patterson D, Brandabur J, Bredfeldt J, Jiranek G, et al., editors. Randomized prospective trial of 50 vs 100 IU BoTox (R) for achalasia-Long term follow-up. Gastroenterology; 1997: WB Saunders Co-Elsivier INC 1600 John kennedy Boulevard, STE 1800, Philadelphia, PE, 19103-2899 USA.

230. Nigam PK, Nigam A. Botulinum Toxin. Indian Journal of Dermatology. 2010;55(1):8-14.

231. Ji J, Lau H, Sheu L, Diamant NE, Gaisano HY. Distinct regional expression of SNARE proteins in the feline oesophagus. Neurogastroenterology and motility : the official journal of the European Gastrointestinal Motility Society. 2002;14(4):383-94.

232. Ma J, Shen J, Lee CA, Elsaidi GA, Smith TL, Walker FO, et al. Gene expression of nAChR, SNAP-25 and GAP-43 in skeletal muscles following botulinum toxin A injection: a study in rats. Journal of orthopaedic research : official publication of the Orthopaedic Research Society. 2005;23(2):302-9.

233. Franklin AL, Petrosyan M, Kane TD. Childhood achalasia: A comprehensive review of disease, diagnosis and therapeutic management. World Journal of Gastrointestinal Endoscopy. 2014;6(4):105-11.

234. Decalmer S, Stovold R, Houghton LA, Pearson J, Ward C, Kelsall A, et al. Chronic cough: relationship between microaspiration, gastroesophageal reflux, and cough frequency. Chest. 2012;142.

235. Harding SM, Schan CA, Guzzo MR, Alexander RW, Bradley LA, Richter JE. Gastroesophageal reflux-induced bronchoconstriction. Is microaspiration a factor? Chest. $1995 ; 108$.

236. Lecoindre P, Richard S. Digestive disorders associated with the chronic obstructive respiratory syndrome of brachycephalic dogs: 30 cases (1999-2001). Revue de médecine vétérinaire. 2004;155(3):141-6.

237. Poncet C, Dupre G, Freiche V, Estrada M, Poubanne Y, Bouvy B. Prevalence of gastrointestinal tract lesions in 73 brachycephalic dogs with upper respiratory syndrome. Journal of Small Animal Practice. 2005;46(6):273-9.

238. Ishikawa T, Sekizawa S-I, Sant'Ambrogio FB, Sant'Ambrogio G. Larynx vs. esophagus as reflexogenic sites for acid-induced bronchoconstriction in dogs. Journal of applied physiology. 1999;86(4):1226-30. 
239. Harris R, Grobman M, Mitchell A, Schachtel J, Rawson N, Bridgette B, et al. Standardizing a Freely-Behaving Canine Videofluoroscopic Swallow Study Protocol to Investigate Dysphagia in Dogs 2016.

240. Mariani G, Boni G, Barreca M, Bellini M, Fattori B, AlSharif A, et al. Radionuclide gastroesophageal motor studies. Journal of Nuclear Medicine. 2004;45(6):1004-28.

241. Radiology ACo. Society of Nuclear Medicine, Society for Pediatric Radiology (2010) ACR-SNM-SPR practice guideline for the performance of gastrointestinal scintigraphy. 2011.

242. Schindelin J, Arganda-Carreras I, Frise E, Kaynig V, Longair M, Pietzsch T, et al. Fiji: an open-source platform for biological-image analysis. Nature methods. 2012;9(7):676.

243. Puranik AD, Nair G, Aggarwal R, Bandyopadhyay A, Shinto A, Zade A. Scintigraphic scoring system for grading severity of gastro-esophageal reflux on 99mTc sulfur colloid gastro-esophageal reflux scintigraphy: A prospective study of 39 cases with pre and post treatment assessment. Indian journal of nuclear medicine : IJNM : the official journal of the Society of Nuclear Medicine, India. 2013;28(2):79-84.

244. Groome M, Cotton JP, Borland M, McLeod S, Johnston DA, Dillon JF. Prevalence of laryngopharyngeal reflux in a population with gastroesophageal reflux. The Laryngoscope. 2007;117(8):1424-8.

245. Koufman JA. Laryngopharyngeal reflux is different from classic gastroesophageal reflux disease. Ear, nose, \& throat journal. 2002;81(9 Suppl 2):7-9.

246. Padhy A, Gopinath P, Sharma S, Prasad A, Arora N, Tiwari D, et al. Radionuclide detection of gastroesophageal reflux in children suffering from recurrent lower respiratory tract infection. The Indian Journal of Pediatrics. 1990;57(4):517-25.

247. Little AF, Cox MR, Martin CJ, Dent J, Franzi SJ, Lavelle R. Influence of posture on transient lower oesophageal sphincter relaxation and gastro-oesophageal reflux in the dog. Journal of gastroenterology and hepatology. 1989;4(1):49-54.

248. Mittal RK, Holloway RH, Penagini R, Blackshaw LA, Dent J. Transient lower esophageal sphincter relaxation. Gastroenterology. 1995;109(2):601-10.

249. Cho YK. How to Interpret Esophageal Impedance pH Monitoring. Journal of neurogastroenterology and motility. 2010;16(3):327-30.

250. Caglar M, Volkan B, Alpar R. Reliability of radionuclide gastroesophageal reflux studies using visual and time-activity curve analysis: inter-observer and intra-observer variation and description of minimum detectable reflux. Nuclear medicine communications. 2003;24(4):421-8.

251. Mousa HM, Rosen R, Woodley FW, Orsi M, Armas D, Faure C, et al. Esophageal impedance monitoring for gastroesophageal reflux. Journal of pediatric gastroenterology and nutrition. 2011;52(2):129-39. 
252. Uslu Kızılkan N, Bozkurt MF, Saltık Temizel IN, Demir H, Yüce A, Caner B, et al. Comparison of multichannel intraluminal impedance-pH monitoring and reflux scintigraphy in pediatric patients with suspected gastroesophageal reflux. World journal of gastroenterology. 2016;22(43):9595-603.

253. Lutsi B, Hirano I. Ambulatory pH Monitoring: New Advances and Indications. Gastroenterology \& Hepatology. 2006;2(11):835-42.

254. Emde C, Garner A, Blum A. Technical aspects of intraluminal pH-metry in man: current status and recommendations. Gut. 1987;28(9):1177.

255. Merati AL, Ulualp SO, Lim HJ, Toohill RJ. Meta-Analysis of Upper Probe Measurements in Normal Subjects and Patients with Laryngopharyngeal Reflux. Annals of Otology, Rhinology \& Laryngology. 2005;114(3):177-82.

256. Patel DA, Blanco M, Vaezi MF. Laryngopharyngeal Reflux and Functional Laryngeal Disorder: Perspective and Common Practice of the General Gastroenterologist. Gastroenterology \& Hepatology. 2018;14(9):512-20.

257. Campagnolo AM, Priston J, Thoen RH, Medeiros T, Assunção AR.

Laryngopharyngeal reflux: diagnosis, treatment, and latest research. International archives of otorhinolaryngology. 2014;18(2):184-91.

258. Johnston N, Knight J, Dettmar PW, Lively MO, Koufman J. Pepsin and carbonic anhydrase isoenzyme III as diagnostic markers for laryngopharyngeal reflux disease. The Laryngoscope. 2004;114(12):2129-34.

259. Sifrim D. Relevance of volume and proximal extent of reflux in gastrooesophageal reflux disease. Gut. 2005;54(2):175-8.

260. Ruth M, Carlsson S, Månsson I, Bengtsson U, Sandberg N. Scintigraphic detection of gastro - pulmonary aspiration in patients with respiratory disorders. Clinical Physiology. 1993;13(1):19-33.

261. Aun MV, Bonamichi-Santos R, Arantes-Costa FM, Kalil J, Giavina-Bianchi P. Animal models of asthma: utility and limitations. Journal of asthma and allergy. 2017;10:293.

262. Myers MJ, Smith ER, Turfle PG. Biomarkers in Veterinary Medicine. Annual Review of Animal Biosciences. 2017;5(1):65-87.

263. Ranieri G, Gadaleta CD, Patruno R, Zizzo N, Daidone MG, Hansson MG, et al. A model of study for human cancer: Spontaneous occurring tumors in dogs. Biological features and translation for new anticancer therapies. Critical reviews in oncology/hematology. 2013;88(1):187-97.

264. Miller I, Presslmayer-Hartler A, Wait R, Hummel K, Sensi C, Eberini I, et al. In between - Proteomics of dog biological fluids. Journal of proteomics. 2014;106:30-45.

265. Kam SY, Hennessy T, Chua SC, Gan CS, Philp R, Hon KK, et al.

Characterization of the human gastric fluid proteome reveals distinct $\mathrm{pH}$-dependent 
protein profiles: implications for biomarker studies. Journal of proteome research. 2011;10(10):4535-46.

266. Hayat JO, Gabieta-Somnez S, Yazaki E, Kang JY, Woodcock A, Dettmar P, et al. Pepsin in saliva for the diagnosis of gastro-oesophageal reflux disease. Gut. 2015;64(3):373-80.

267. Krishnan U, Mitchell JD, Messina I, Day AS, Bohane TD. Assay of tracheal pepsin as a marker of reflux aspiration. J Pediatr Gastroenterol Nutr. 2002;35(3):303-8.

268. Ocak E, Kubat G, Yorulmaz I. Immunoserologic pepsin detection in the saliva as a non-invasive rapid diagnostic test for laryngopharyngeal reflux. Balkan medical journal. 2015;32(1):46-50.

269. Fernandes M, Rosa N, Esteves E, Correia MJ, Arrais J, Ribeiro P, et al. CanisOme--The protein signatures of Canis lupus familiaris diseases. Journal of proteomics. 2016;136:193-201.

270. Wright JL, Cosio M, Churg A. Animal models of chronic obstructive pulmonary disease. american journal of physiology-lung cellular and molecular physiology. 2008;295(1):L1-L15.

271. Gharaee-Kermani M, Ullenbruch M, Phan SH. Animal models of pulmonary fibrosis. Fibrosis Research: Methods and Protocols. 2005:251-9.

272. Norris Reinero CR, Decile KC, Berghaus RD, Williams KJ, Leutenegger CM, Walby WF, et al. An experimental model of allergic asthma in cats sensitized to house dust mite or bermuda grass allergen. International archives of allergy and immunology. 2004;135(2):117-31.

273. Williams K, Roman J. Studying human respiratory disease in animals-role of induced and naturally occurring models. The Journal of pathology. 2016;238(2):220-32.

274. Lilja-Maula LI, Palviainen MJ, Heikkilä HP, Raekallio MR, Rajamäki MM. Proteomic analysis of bronchoalveolar lavage fluid samples obtained from West Highland White Terriers with idiopathic pulmonary fibrosis, dogs with chronic bronchitis, and healthy dogs. American journal of veterinary research. 2013;74(1):14854.

275. Paulo JA, Lee LS, Wu B, Repas K, Banks PA, Conwell DL, et al. Proteomic analysis of endoscopically (endoscopic pancreatic function test) collected gastroduodenal fluid using in-gel tryptic digestion followed by LC-MS/MS. Proteomics Clinical applications. 2010;4(8-9):715-25.

276. Knight J, Lively MO, Johnston N, Dettmar PW, Koufman JA. Sensitive pepsin immunoassay for detection of laryngopharyngeal reflux. The Laryngoscope. 2005;115(8):1473-8.

277. Lambert E. Secretion Management. Pediatric Dysphagia: Springer; 2018. p. 25569. 
278. Yandrapu H, Marcinkiewicz M, Sarosiek I, Sarosiek J, Poplawski C, Han K, et al. Role of saliva in esophageal defense: implications in patients with nonerosive reflux disease. The American journal of the medical sciences. 2015;349(5):385-91.

279. Hu S, Loo JA, Wong DT. Human body fluid proteome analysis. Proteomics. 2006;6(23):6326-53.

280. Frantzi M, Bhat A, Latosinska A. Clinical proteomic biomarkers: relevant issues on study design \& technical considerations in biomarker development. Clinical and translational medicine. 2014;3(1):7.

281. Borrebaeck CA, Wingren C. Transferring proteomic discoveries into clinical practice. Expert review of proteomics. 2009;6(1):11-3.

282. Banks RE. Preanalytical influences in clinical proteomic studies: raising awareness of fundamental issues in sample banking. Clinical Chemistry; 2008.

283. Mischak H, Apweiler R, Banks RE, Conaway M, Coon J, Dominiczak A, et al. Clinical proteomics: a need to define the field and to begin to set adequate standards. PROTEOMICS-Clinical Applications. 2007;1(2):148-56.

284. Freeman LM, Rush JE, Farabaugh AE, Must A. Development and evaluation of a questionnaire for assessing health-related quality of life in dogs with cardiac disease. Journal of the American Veterinary Medical Association. 2005;226(11):1864-8.

285. Spinou A, Birring SS. An update on measurement and monitoring of cough: what are the important study endpoints? Journal of thoracic disease. 2014;6(Suppl 7):S728.

286. Grobman M, Reinero C. Investigation of Neurokinin - 1 Receptor Antagonism as a Novel Treatment for Chronic Bronchitis in Dogs. Journal of veterinary internal medicine. 2016;30(3):847-52.

287. Verschelden P, Cartier A, L'Archeveque J, Trudeau C, Malo JL. Compliance with and accuracy of daily self-assessment of peak expiratory flows (PEF) in asthmatic subjects over a three month period. The European respiratory journal. 1996;9(5):880-5.

288. Martinek J, Klco P, Vrabec M, Zatko T, Tatar M, Javorka M. Cough sound analysis. Acta Medica Martiniana. 2013;13(Supplement 1):15-20.

289. Subburaj S, Parvez L, Rajagopalan TG. Methods of recording and analysing cough sounds. Pulmonary pharmacology. 1996;9(5-6):269-79.

290. Knocikova J, Korpas J, Vrabec M, Javorka M. Wavelet analysis of voluntary cough sound in patients with respiratory diseases. J Physiol Pharmacol. 2008;59:331-40.

291. Abaza AA, Day JB, Reynolds JS, Mahmoud AM, Goldsmith WT, McKinney WG, et al. Classification of voluntary cough sound and airflow patterns for detecting abnormal pulmonary function. Cough (London, England). 2009;5(8):9284.

292. Chisnell HK, Pardo AD. Long-term outcome, complications and disease progression in 23 dogs after placement of tracheal ring prostheses for treatment of extrathoracic tracheal collapse. Veterinary surgery : VS. 2015;44(1):103-13. 
293. Johnson LR, McKiernan BC. Diagnosis and medical management of tracheal collapse. Seminars in veterinary medicine and surgery (small animal). 1995;10(2):101-8.

294. Eccles R. Importance of placebo effect in cough clinical trials. Lung. 2010;188 Suppl 1:S53-61.

295. Scheuermann TS, Richter KP, Rigotti NA, Cummins SE, Harrington KF, Sherman SE, et al. Accuracy of self - reported smoking abstinence in clinical trials of hospital - initiated smoking interventions. Addiction. 2017;112(12):2227-36.

296. Chang AB, Newman RG, Carlin JB, Phelan PD, Robertson CF. Subjective scoring of cough in children: parent-completed vs child-completed diary cards vs an objective method. The European respiratory journal. 1998;11(2):462-6.

297. Morey MJ, Cheng AC, McCallum GB, Chang AB. Accuracy of cough reporting by carers of Indigenous children. Journal of paediatrics and child health. 2013;49(3):E199-203.

298. Leconte S, Liistro G, Lebecque P, Degryse JM. The objective assessment of cough frequency: accuracy of the LR102 device. Cough (London, England). 2011;7:11.

299. Krajnik M, Damps-Konstanska I, Gorska L, Jassem E. A portable automatic cough analyser in the ambulatory assessment of cough. BioMedical Engineering OnLine. 2010;9:17.

300. Mesaros A, Heittola T, Eronen A, Virtanen T, editors. Acoustic event detection in real life recordings. 2010 18th European Signal Processing Conference; 2010: IEEE.

301. Doherty M, Wang L, Donague S, Pearson M, Downs P, Stoneman S, et al. The acoustic properties of capsaicin-induced cough in healthy subjects. European Respiratory Journal. 1997;10(1):202-7.

302. Fontana GA, Widdicombe J. What is cough and what should be measured?

Pulmonary Pharmacology \& Therapeutics. 2007;20(4):307-12.

303. Xiao Y, Carlson D, Boris L, Mabary J, Lin Z, Nicodème F, et al. The acoustic cough monitoring and manometric profile of cough and throat clearing. Diseases of the esophagus : official journal of the International Society for Diseases of the Esophagus / ISDE. 2014;27(1):5-12.

304. Molina-Pallete GM, Ray C. Chronic Cough Of Unknown Primary. B39 Cough: Rare and Common: American Thoracic Society; 2017. p. A3345-A.

305. Smith J. Ambulatory methods for recording cough. Pulm Pharmacol Ther. 2007;20(4):313-8.

306. Pavesi L, Subburaj S, Porter-Shaw K. Application and validation of a computerized cough acquisition system for objective monitoring of acute cough: a metaanalysis. Chest. 2001;120(4):1121-8.

307. Smith J, Woodcock A. New developments in the objective assessment of cough. Lung. 2008;186(1):48-54. 
308. Irwin RS, Zawacki JK, Wilson MM, French CT, Callery MP. Chronic cough due to gastroesophageal reflux disease: failure to resolve despite total/near-total elimination of esophageal acid. Chest. 2002;121.

309. Palombini BC, Villanova CA, Araujo E, Gastal OL, Alt DC, Stolz DP, et al. A pathogenic triad in chronic cough: asthma, postnasal drip syndrome, and gastroesophageal reflux disease. Chest. 1999;116(2):279-84.

310. Bonadio C, Pollard RE, Dayton PA, Leonard C, Marks SL. Effects of body positioning on swallowing and esophageal transit in healthy dogs. Journal of veterinary internal medicine. 2009;23(4):801-5.

311. Cheney DM, Marks SL, Pollard RE. Effect of bolus size on deguttition and esophageal transit in healthy dogs Veterinary Radiology \& Ultrasound. 2016.

312. Pollard RE. Imaging Evaluation of Dogs and Cats with Dysphagia. ISRN Veterinary Science. 2012;2012:15.

313. Grobman ME, Hutcheson KD, Lever TE, Mann FA, Reinero CR. Mechanical dilation, botulinum toxin A injection, and surgical myotomy with fundoplication for treatment of lower esophageal sphincter achalasia-like syndrome in dogs. Journal of veterinary internal medicine. 2019.

314. Shah K, Guarderas J, Krishnaswamy G. Aspiration-induced pulmonary syndromes. Annals of Allergy, Asthma \& Immunology. 2016;117(5):479-82.

315. Piccione JC, McPhail GL, Fenchel MC, Brody AS, Boesch RP. Bronchiectasis in chronic pulmonary aspiration: Risk factors and clinical implications. Pediatric pulmonology. 2012;47(5):447-52.

316. Matsuse T, Fukuchi Y, Oka T, Kida K. Importance of diffuse aspiration bronchiolitis caused by chronic occult aspiration in the elderly. Chest. 1996;110(5):128993.

317. Hadda V, Khilnani GC. Lipoid pneumonia: an overview. Expert review of respiratory medicine. 2010;4(6):799-807.

318. Lou Z. Assessment of laryngopharyngeal reflux and the shape of the Eustachian tube should be considered in chronic rhinosinusitis with nasal polyps and chronic otitis media. European archives of oto-rhino-laryngology : official journal of the European Federation of Oto-Rhino-Laryngological Societies (EUFOS) : affiliated with the German Society for Oto-Rhino-Laryngology - Head and Neck Surgery. 2017;274(12):4265-6.

319. Gaynor EB. Gastroesophageal reflux as an etiologic factor in laryngeal complications of intubation. The Laryngoscope. 1988;98(9):972-9.

320. Stadler K, Hartman S, Matheson J, O'BRIEN R. Computed tomographic imaging of dogs with primary laryngeal or tracheal airway obstruction. Veterinary Radiology \& Ultrasound. 2011;52(4):377-84. 
321. Carminato A, Vascellari M, Zotti A, Fiorentin P, Monetti G, Mutinelli F. Imaging of exogenous lipoid pneumonia simulating lung malignancy in a dog. The Canadian veterinary journal $=$ La revue veterinaire canadienne. $2011 ; 52(3): 310-2$.

322. Holman SD, Campbell-Malone R, Ding P, Gierbolini-Norat EM, Griffioen AM, Inokuchi $\mathrm{H}$, et al. Development, reliability, and validation of an infant mammalian penetration-aspiration scale. Dysphagia. 2013;28(2):178-87. 


\section{VITA}

Megan Grobman is a diplomate of the American College of Veterinary Internal Medicine with special interests in respiratory medicine, dysphagia, and respiratory biometrics. She speaks nationally and internationally on topics related to the intersection between respiratory and gastrointestinal disease (aerodigestive disorders). She completed specialty training, and a master's degree at the University of Missouri. She hopes to continue her work on aerodigestive disease as a clinician scientist at a veterinary teaching hospital. 